NATIONAL LABORATORY

\title{
Next Generation Nuclear Plant GAP Analysis Report
}

\section{July 2008}

Prepared by

S. J. Ball

R\&D Staff Member

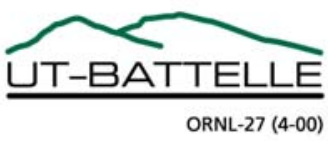




\section{DOCUMENT AVAILABILITY}

Reports produced after January 1, 1996, are generally available free via the U.S. Department of Energy (DOE) Information Bridge.

Web site http://www.osti.gov/bridge

Reports produced before January 1, 1996, may be purchased by members of the public from the following source.

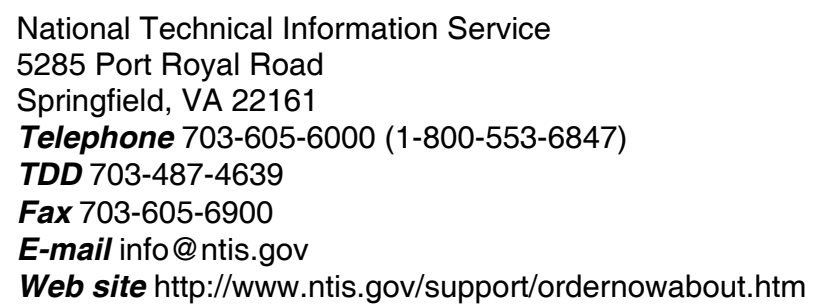

Reports are available to DOE employees, DOE contractors, Energy Technology Data Exchange (ETDE) representatives, and International Nuclear Information System (INIS) representatives from the following source.

Office of Scientific and Technical Information

P.O. Box 62

Oak Ridge, TN 37831

Telephone 865-576-8401

Fax 865-576-5728

E-mail reports@osti.gov

Web site http://www.osti.gov/contact.html

This report was prepared as an account of work sponsored by an agency of the United States Government. Neither the United States Government nor any agency thereof, nor any of their employees, makes any warranty, express or implied, or assumes any legal liability or responsibility for the accuracy, completeness, or usefulness of any information, apparatus, product, or process disclosed, or represents that its use would not infringe privately owned rights. Reference herein to any specific commercial product, process, or service by trade name, trademark, manufacturer, or otherwise, does not necessarily constitute or imply its endorsement, recommendation, or favoring by the United States Government or any agency thereof. The views and opinions of authors expressed herein do not necessarily state or reflect those of the United States Government or any agency thereof. 
Nuclear Science \& Technology Division

\title{
NEXT GENERATION NUCLEAR PLANT GAP ANALYSIS REPORT
}

\author{
S. J. Ball, ${ }^{1}$ T. D. Burchell, W. R. Corwin, S. E. Fisher \\ C. W. Forsberg, ${ }^{2}$ R. N. Morris, D. L. Moses \\ Prepared for the \\ U.S. Nuclear Regulatory Commission \\ by \\ Oak Ridge National Laboratory
}

\footnotetext{
${ }^{1}$ Corresponding Author

${ }^{2}$ Massachusetts Institute of Technology, Cambridge, MA 02139
}

Date Published: July 2008

Prepared by

OAK RIDGE NATIONAL LABORATORY

Oak Ridge, Tennessee 37831-6283

managed by

UT-BATTELLE, LLC

for the

U.S. DEPARTMENT OF ENERGY

under contract DE-AC05-00OR22725 



\section{CONTENTS}

Page_Toc203537209

LIST OF FIGURES vii

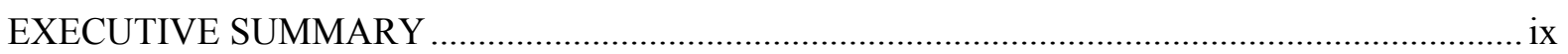

ABBREVIATIONS AND ACRONYMS.............................................................................

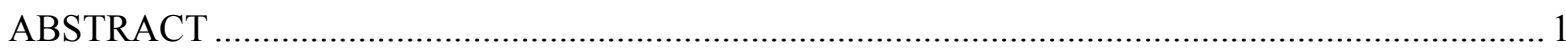

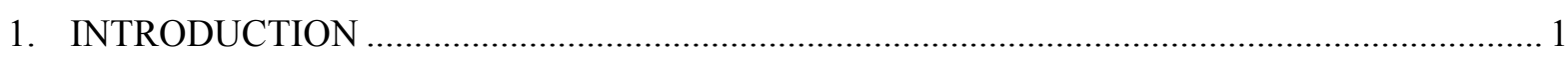

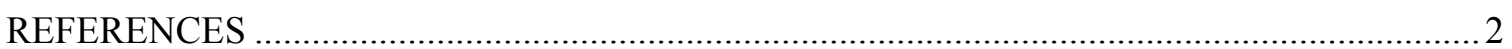

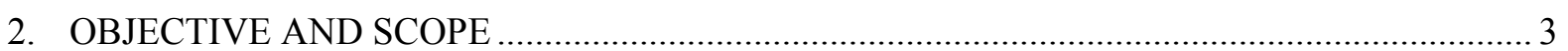

3. ACCIDENT AND THERMAL-FLUIDS (T/F) PHENOMENA .................................................. 5

3.1 MAJOR PHENOMENA AND PROCESSES OF INTEREST ............................................. 5

3.2 EXPERIMENTAL AND ANALYTICAL MODELING ASPECTS OF PHENOMENA-

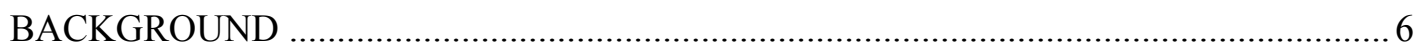

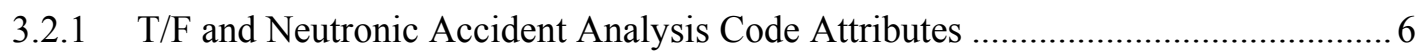

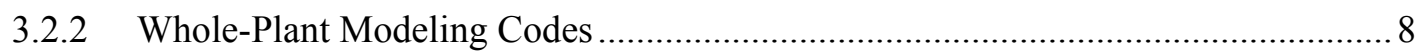

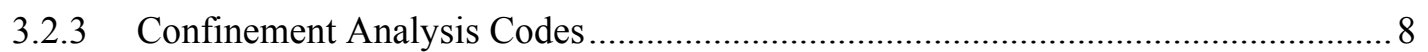

3.2.4 Codes Dealing with Chemical Reactions ............................................................. 9

3.3 CLOSING THE GAPS—STAGES OF MODEL DEVELOPMENT ................................... 10

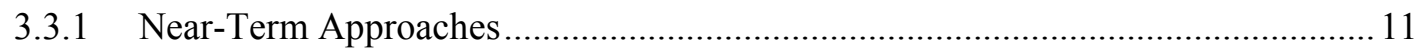

3.3.2 Intermediate- and Long-Term Approaches ....................................................... 13

3.3.3 Summary Descriptions and Evaluations of Selected Existing Code

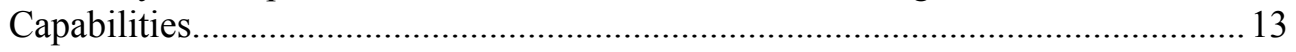

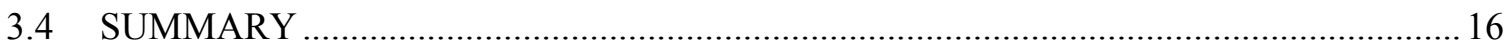

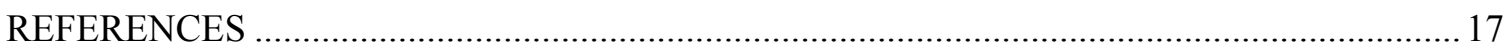

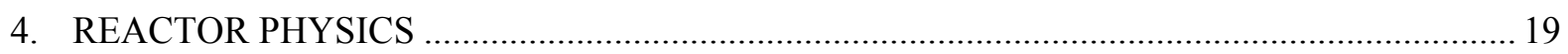

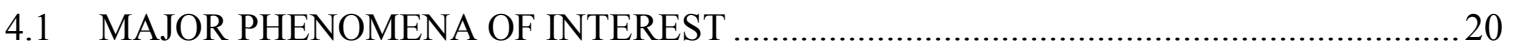

4.1.1 Background in Selection of Phenomena ....................................................................2 20

4.1.2 Design Aspects That Impact Neutronic Phenomena .................................................2

4.2 EXPERIMENTAL AND ANALYTICAL MODELING ASPECTS OF PHENOMENA .. 25

4.3 CLOSING THE GAPS-STAGES OF MODEL DEVELOPMENT .................................27

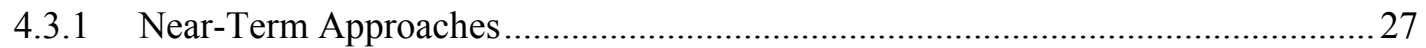

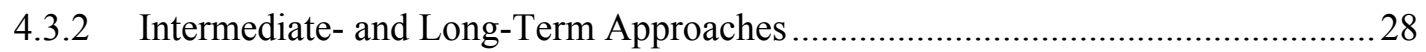

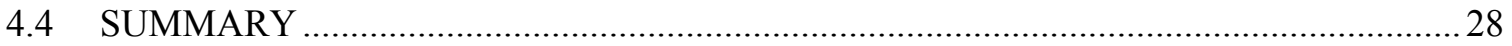

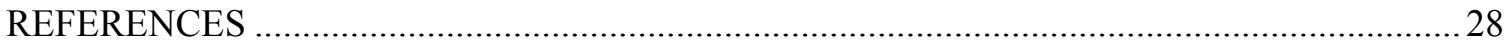




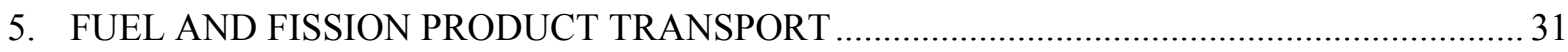

5.1 FISSION PRODUCT AND RADIOACTIVE MATERIALS TRANSPORT

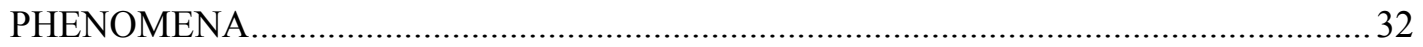

5.2 EXPERIMENTAL AND ANALYTICAL MODELING ASPECTS OF PHENOMENA .. 32

5.2.1 Background and History of FPT Model Development ........................................... 32

5.2.2 Physical Models of Phenomena and Supporting Analytical Methods ..................... 34

5.2.3 Brief Summary of Phenomena Models ................................................................ 36

5.2.4 Relevant Material or Component Data Over the Range of Interest and the

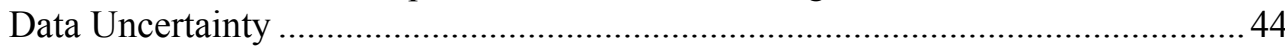

5.2.5 The Reactor Component and Confinement/Containment Configuration and Their Relative Roles in the Safety Case ................................................................... 45

5.2.6 Computational Software or Other Methods for Determining the Quantitative

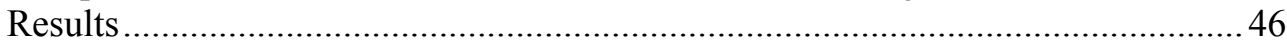

5.2.7 Integral Testing Over a Wide Range of Conditions to Support the Computational Methods and Their Uncertainty....................................................4 47

5.3 CLOSING THE GAPS—STAGES OF MODEL DEVELOPMENT ................................. 47

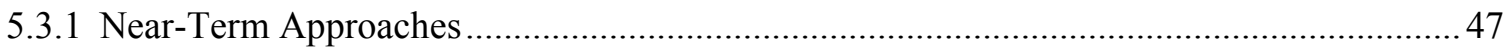

5.3.2 Intermediate- and Long-Term Approaches ...................................................... 49

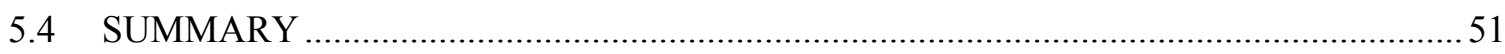

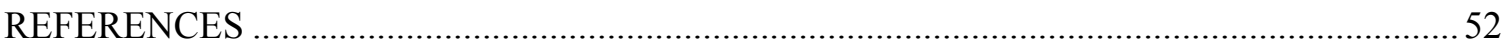

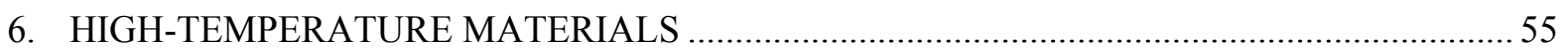

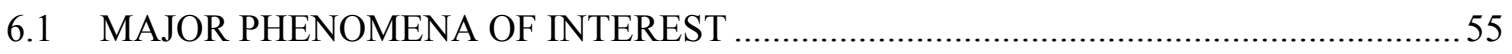

6.1.1 Phenomena Ranked Importance - High, Knowledge - Low …..............................55

6.1.2 Phenomena Ranked Importance-High, Knowledge-Medium .............................58

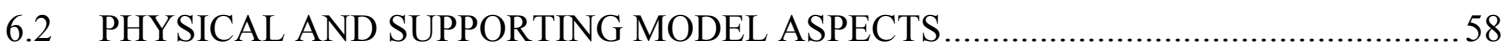

6.3 CLOSING THE GAPS—STAGES OF MODEL DEVELOPMENT ...................................59

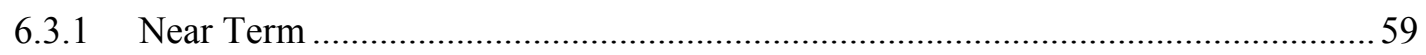

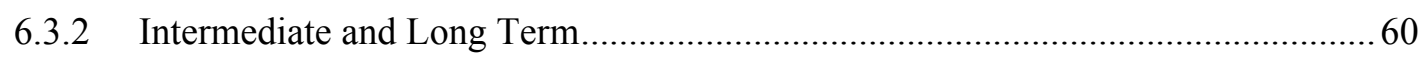

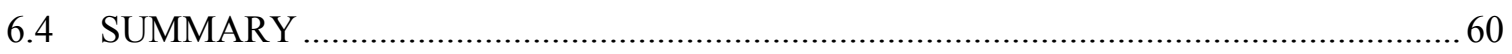

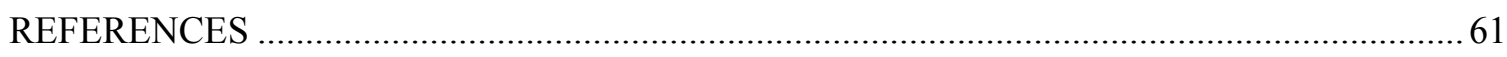

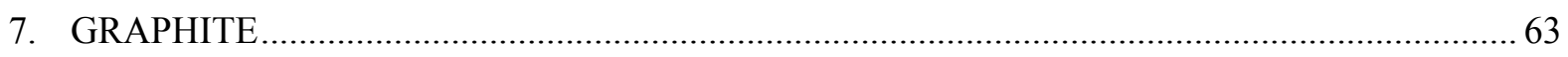

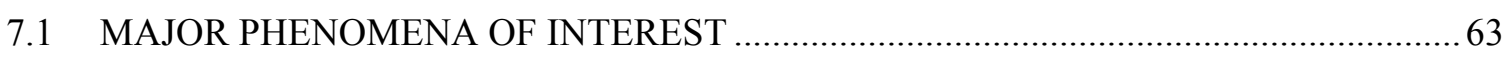

7.1.1 Phenomena Ranked Importance-High, Knowledge — Low .................................63

7.1.2 Phenomena Ranked Importance-High, Knowledge-Medium ............................. 64

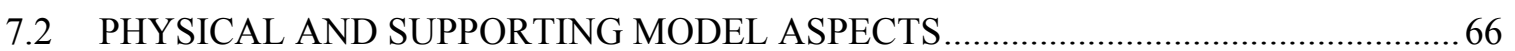

7.3 CLOSING THE GAPS—STAGES OF MODEL DEVELOPMENT ..................................66 


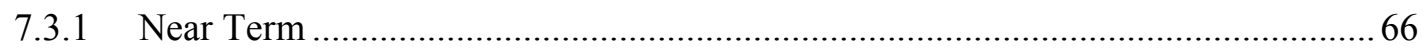

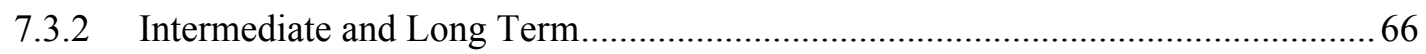

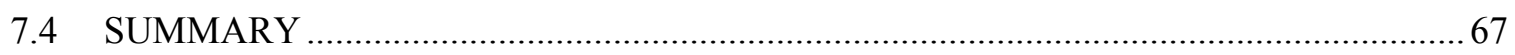

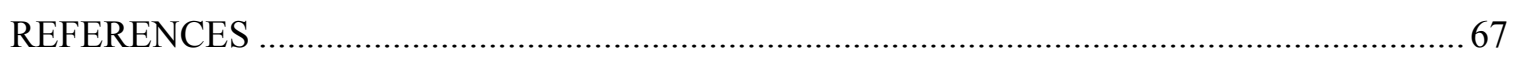

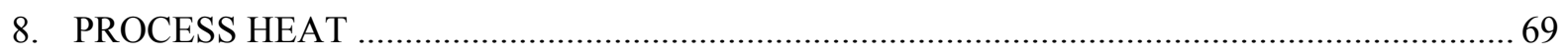

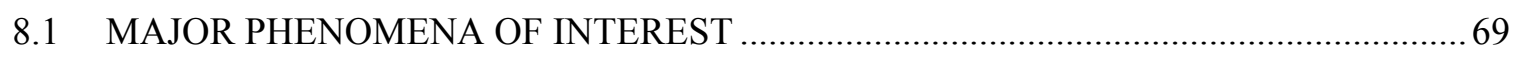

8.2 EXPERIMENTAL AND ANALYTICAL MODELING OF PHENOMENA …................. 70

8.3 CLOSING THE GAPS—STAGES OF MODEL DEVELOPMENT ................................... 70

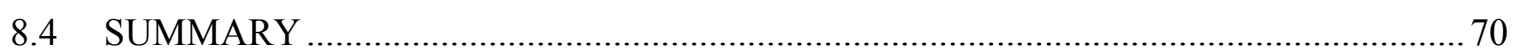

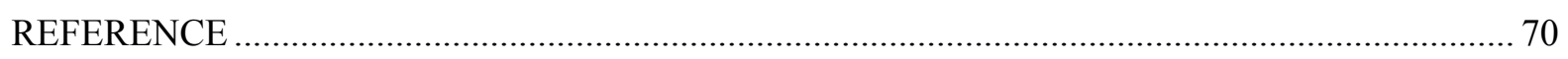





\section{LIST OF FIGURES}

Figure

Page

$1 \quad$ NGNP reactor core options_-Prismatic (L) and Pebble Bed (R) ........................................ 2

2 TINTE code modeling block diagram as developed by INET …............................................ 14 



\section{EXECUTIVE SUMMARY}

This report follows up on recent NRC-sponsored phenomena identification and ranking table (PIRT) exercises for the next generation nuclear plant (NGNP) and is intended to identify the significant "gaps" between what is needed and what is already available to NRC to adequately assess NGNP safety characteristics. Building on the PIRT efforts, this task goes a step further by incorporating evaluations of accident sequences and risk to determine important gaps in the knowledge base and further to recommend how these gaps might be addressed. Report sections are typically organized to first provide a background and summary of what is needed, next identify what data and tools are available, and finally describe the gaps. This information is of interest to NRC assessments of the confirmatory research and development (R\&D) needs for NGNP licensing. Gap analyses are based in part on the set of phenomena determined in the PIRT processes to be of high importance and have a low or medium knowledge level, with evaluations of accident sequence risk factored in also.

The scope of this report covers six areas or categories, where many phenomena involved are cross-cutting. They are

1. accidents and thermal fluids,

2. reactor physics and neutronics (including criticality calculations and experiments),

3. fuel performance and fission product transport (FPT) and dose,

4. high-temperature materials (metallic),

5. graphite, and

6. process heat for hydrogen production.

Some recommendations are categorized for either near- or long-term implementation. This is, in part, in recognition of the fact that the NGNP design is still in the early stages of definition and development, and some emphasis on research needs is expected to change as the design matures.

The most significant gaps in the accident and thermal-fluids area have been identified as

1. core coolant bypass flow (normal operation),

2. core effective thermal conductivity [depressurized loss of forced circulation (D-LOFC)],

3. reactor cavity cooling system (RCCS) performance [during loss of forced circulation (LOFC)], and

4. confinement with reactor cavity air ingress (air ingress accident).

In the physics or neutronics area, there were no gaps found to be of high importance to safety; however, there were a number of items identified and described in some detail that would require especially careful attention to detail within the normal design and testing program.

The major FPT phenomena of interest identified in the PIRT exercise are also covered in this report. The primary issues relate to current uncertainties in confinement design (which have a major impact on "gap priorities"), fission product (FP) releases via normal helium leakage, and dust-borne releases and deleterious effects of mechanical shock and vibrations during rapid discharge in postulated D-LOFC accidents which could cause additional releases of FPs that would have otherwise been retained within the primary system.

The following significant gaps were identified in the NGNP reactor high-temperature materials area (other than graphite) and corresponded directly to those identified in the PIRT exercise: 
- for the reactor pressure vessel (RPV), crack initiation and subcritical crack growth, surface emissivity, and loss of desired surface layer and other property degradation;

- aging and fatigue leading to degradation of insulation materials;

- intermediate heat exchanger (IHX) crack initiation and propagation;

- structural design methods for control rod composites;

- in-vessel surface emissivities and irradiation-induced creep;

- structural design and fabrication of composites; and

- valve failure in high-temperature conditions.

Significant gaps identified for graphite components were found in the following areas, also corresponding directly to those identified by the PIRT panel:

- graphite supply uncertainties and inconsistencies;

- confirmatory data for new grades to be used in NGNP;

- irradiation creep data and effect of creep on properties;

- consensus design codes and standards;

- extension of current theoretical performance models to higher doses and temperatures;

- development of whole-core structural models and nondestructive examination (NDE) methods; and

- analytical models for oxidation, property changes, and dimensional changes with creep induced by irradiation.

For the high-temperature process heat systems (for hydrogen production), model development will be required once the hydrogen plant design is specified. The application of engineering judgment and lessons learned from nonnuclear chemical plant experience is needed to eliminate or minimize the possibility of accidents affecting both the reactor and the process plant. Potential phenomena resulting from process plant interface with the reactor are highly design dependent. As was also concluded in the PIRT study, the design selections should be guided to eliminate or minimize the phenomena that can adversely affect reactor safety. 


\section{ABBREVIATIONS AND ACRONYMS}

AGR

ANL

ANS

AOO

ASME

ASTM

ATWS

AVR

$\mathrm{B} \& \mathrm{PV}$

BOP

CFR

CFD

CHE

CRP

CTE

D-LOFC

DBA

DDN

DOE

$\mathrm{EAB}$

FP

FPT

GA

GRS

GRSAC

GT-MHR

HEU

HTGR

HTR-10

HTR-PM

HTTR

HVAC

IAEA

IHX

INET

INL

IRSN

KAERI

LEU
Advanced Gas Reactor (British)

Argonne National Laboratory

American Nuclear Society

anticipated operational occurrence

American Society of Mechanical Engineers

American Society for Testing and Materials

anticipated transients without scram

Atomgeneinschaft Versuchs Reaktor

Boiler and Pressure Vessel (ASME Code)

balance of plant

Code of Federal Regulations

computational fluid dynamics

compact heat exchanger

Coordinated Research Project (IAEA)

coefficient of thermal expansion

depressurized loss-of-forced circulation

design basis accident

design data need

Department of Energy

exclusion area boundary

fission product

fission product transport

General Atomics

Gesellschaft für Anlagen und Reaktorsicherheit mbH (Germany)

Graphite Reactor Severe Accident Code

gas-turbine-modular helium reactor

highly enriched uranium

high-temperature gas-cooled reactor

high-temperature reactor (10 MW-China)

high-temperature reactor - power module (China)

high-temperature engineering test reactor (Japan)

heating, ventilating, and air conditioning

International Atomic Energy Agency

intermediate heat exchanger

Institute of Nuclear Energy Technology at Tsinghua University (China)

Idaho National Laboratory (formerly INEL and INEEL)

L'Institut de radioprotection et de sûreté nucléaire (France)

Korean Atomic Energy Research Institute

low-enriched uranium 


\begin{tabular}{|c|c|}
\hline LOCA & loss-of-coolant accident \\
\hline LOFC & loss-of-forced circulation \\
\hline LWR & light-water reactor \\
\hline MHTGR & modular high-temperature gas-cooled reactor \\
\hline $\mathrm{mR}$ & milli-Rem \\
\hline MTC & moderator temperature-dependent reactivity coefficient \\
\hline NDE & nondestructive examination \\
\hline NGNP & next generation nuclear plant \\
\hline NRC & Nuclear Regulatory Commission (U. S.) \\
\hline NRG & Nuclear Research Group (Netherlands) \\
\hline ORNL & Oak Ridge National Laboratory \\
\hline OSTI & Office of Scientific and Technical Information \\
\hline OUO & official use only \\
\hline P-LOFC & pressurized loss-of-forced circulation \\
\hline PAG & protective action guidelines \\
\hline PBMR & pebble-bed modular reactor (Pty. Ltd.) \\
\hline PBR & pebble-bed reactor \\
\hline PHX & process heat exchanger \\
\hline PIRT & phenomena identification and ranking table \\
\hline PMR & prismatic core modular reactor \\
\hline PWHT & post-weld heat treatment \\
\hline $\mathrm{R}$ & rem \\
\hline R\&D & research and development \\
\hline RCCS & reactor cavity cooling system \\
\hline RIA & reactivity insertion accident \\
\hline RPV & reactor pressure vessel \\
\hline RSA & Republic of South Africa \\
\hline RSS & reserve shutdown system \\
\hline SAS & small absorber spheres \\
\hline SCS & shutdown cooling system \\
\hline SER & safety evaluation report \\
\hline SRS & Safety Report Series (IAEA) \\
\hline $\mathrm{SSCs}$ & structures, systems, and components \\
\hline $\mathrm{T} / \mathrm{F}$ & thermal-fluids \\
\hline THTR & thorium high-temperature reactor \\
\hline TRISO & tri-structural isotropic \\
\hline UHTREX & Ultra High Temperature Reactor Experiment \\
\hline $\mathrm{V} \& \mathrm{~V}$ & verification and validation \\
\hline VHTGR & very high temperature gas-cooled reactor \\
\hline
\end{tabular}




\begin{abstract}
As a follow-up to the phenomena identification and ranking table (PIRT) studies conducted recently by NRC on next generation nuclear plant (NGNP) safety, a study was conducted to identify the significant "gaps" between what is needed and what is already available to adequately assess NGNP safety characteristics. The PIRT studies focused on identifying important phenomena affecting NGNP plant behavior, while the gap study gives more attention to off-normal behavior, uncertainties, and event probabilities under both normal operation and postulated accident conditions. Hence, this process also involved incorporating more detailed evaluations of accident sequences and risk assessments. This study considers thermal-fluid and neutronic behavior under both normal and postulated accident conditions, fission product transport (FPT), high-temperature metals, and graphite behavior and their effects on safety. In addition, safety issues related to coupling process heat (hydrogen production) systems to the reactor are addressed, given the limited design information currently available. Recommendations for further study, including analytical methods development and experimental needs, are presented as appropriate in each of these areas.
\end{abstract}

\title{
1. INTRODUCTION
}

The physical processes governing the behavior of high-temperature gas-cooled reactors (HTGRs) are complex and in many ways differ considerably from those of light-water reactors (LWRs). Part of the process of evaluating HTGR safety characteristics is the understanding of phenomena involved, along with the models developed to predict behavior in both normal and accident situations. Another significant part of the evaluation process is identifying those areas in which gaps remain between what is needed to conduct comprehensive safety analysis vs what is available to the analyst, including operationally validated models with adequate supporting data. Reductions in the uncertainties in the analysis models and data would result in a reduction of the thermal margins required to make the safety case. Gap reduction assessments are addressed for both near- and long-term needs.

The phenomena identification and ranking table (PIRT) process has been applied by the U.S. Nuclear Regulatory Commission (NRC) to the current body of information about the U.S. Department of Energy's (DOE's) Next Generation Nuclear Plant (NGNP_assumed to be a modular HTGR), considering both the prismatic and pebble-bed core design options.

Figure 1 (below) shows examples of the two types of NGNP reactor cores-prismatic and pebble bed. 

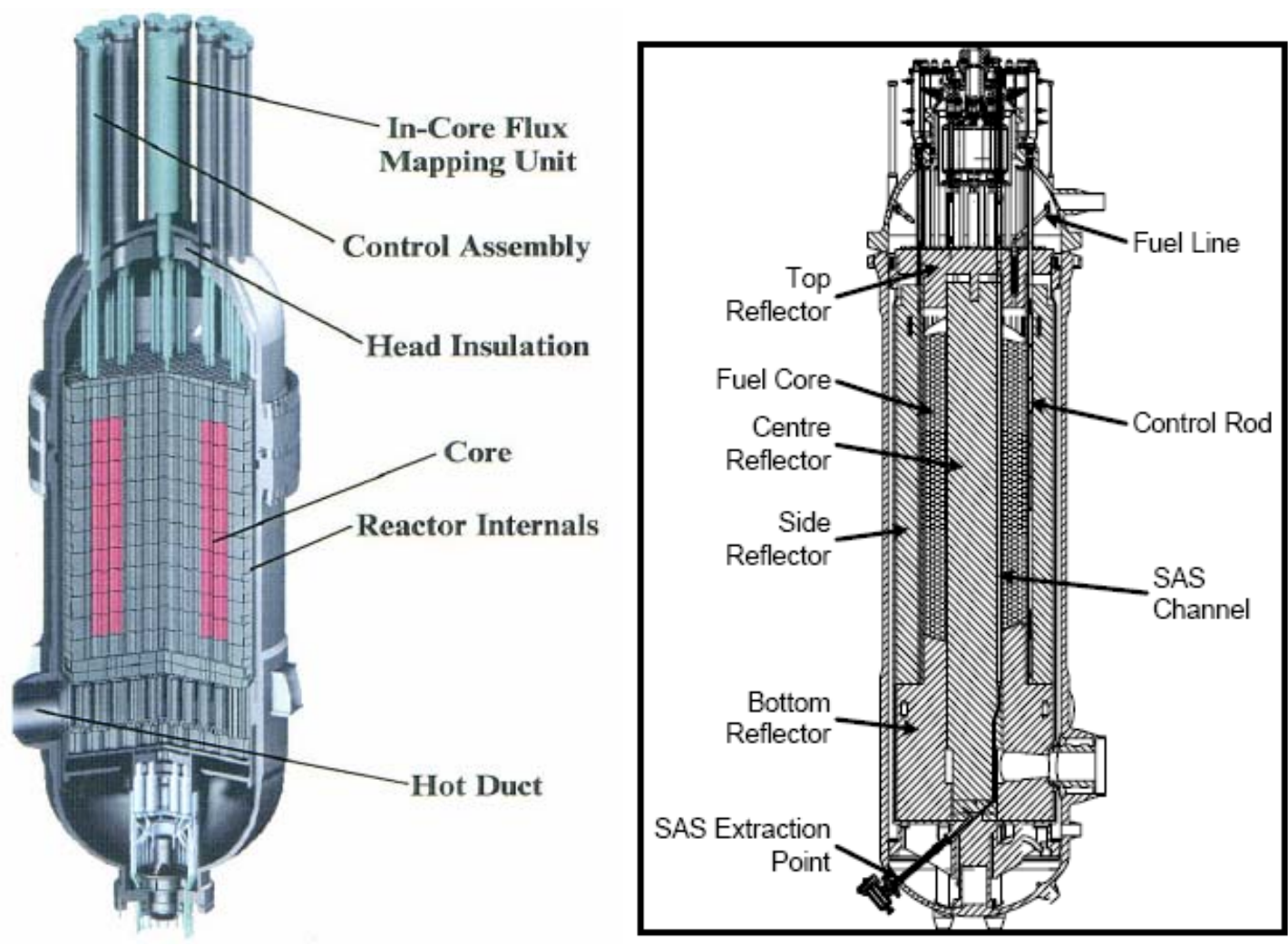

Fig. 1. NGNP reactor core options—Prismatic (L) and Pebble Bed (R).

Five PIRT teams recently evaluated phenomena in the following areas: accidents, including thermal fluids (T/F) and neutronics; fission product transport (FPT) and dose; high-temperature metallic materials; graphite; and high-temperature process heat utilization for hydrogen production. This PIRT activity is documented in the six volumes of NUREG/CR-6944 [1-1]. Prior to that exercise, a PIRT process was conducted for the key phenomena pertinent to TRISO-coated particle fuel (to be used in the NGNP), documented in NUREG/CR-6844 [1-2]. Both activities provided assessments of importance and knowledge level rankings for significant phenomena.

The reader is encouraged to read the PIRT main reports as well as the additional volumes providing more details of the individual PIRTs.

\section{REFERENCES}

1-1. S. J. Ball and S. E. Fisher, Next Generation Nuclear Plant Phenomena Identification and Ranking Tables (PIRTs)_Volume 1: Main Report, NUREG/CR-6944, Vol. 1 (ORNL/TM2007/147, Vol. 1), Oak Ridge National Laboratory, March 2008 (and additional volumes).

1-2. $\quad$ R. N. Morris et al., TRISO-Coated Particle Fuel Phenomenon Identification and Ranking Tables (PIRTs) for Fission Product Transport Due to Manufacturing, Operations, and Accidents-Main Report, NUREG/CR-6844, Vol. 1, Nuclear Regulatory Commission, Washington, DC, July 2004 (and additional volumes). 


\section{OBJECTIVE AND SCOPE}

Building on previous PIRT studies, the objective of this report is to go a step further by incorporating evaluations of accident sequences and risk to identify gaps in the knowledge base and to recommend how significant gaps should be addressed. Report sections are typically organized to first provide a background and summary of what is needed, next identify what options are available, and finally describe what is missing ("the gap") and how to fill it. This information is critical to NRC assessment of the confirmatory research and development (R\&D) needs for NGNP licensing. Gaps in the knowledge base and suggested approaches for filling them will be based in part on the set of phenomena determined in the PIRT processes to be of high importance and have a low or medium knowledge level. Evaluations of accident sequence risk will be factored in also.

The scope of this report covers six areas or categories of phenomena, processes, or characteristics (hereafter referred to as "phenomena"). Unquestionably, many phenomena are cross-cutting, and these cross-cuts are addressed in individual sections. The following categorization is made for the purposes of report organization to address the important phenomena. They are

1. accidents and thermal fluids,

2. reactor physics and neutronics (including criticality calculations and experiments),

3. fuel performance and FPT and dose,

4. high-temperature materials (metallic),

5. graphite, and

6. process heat for hydrogen production.

It is recognized that helium purification and spent fuel storage systems could also become source terms in certain events; however, they are not covered in this report. Some recommendations are categorized for either near- or long-term implementation. This is due in part to the fact that the NGNP is still in the early stages of definition and development, and the emphasis on research needs is expected to change as the design matures and becomes more specific. 



\section{ACCIDENT AND THERMAL-FLUIDS (T/F) PHENOMENA}

This section provides a background on modular HTGR accident and T/F characteristics, emphasizing the phenomena that need to be addressed in accident codes. Most of these issues are addressed adequately in existing codes used by the world's major HTGR programs, but there are some areas where, in general, more work is needed. These areas will be identified as gaps, and some recommendations on how the gaps might be filled will be offered where applicable.

This section will cover topics based on challenges to four fundamental safety functions, as follows:

- heat removal,

- reactivity control,

- confinement of radioactivity, and

- control of chemical attacks.

Some neutronics and FPT issues are also identified where appropriate to the accident analysis, but Sects. 4 and 5 will address those specific areas in more detail.

Safety-related heat removal involves timely and sufficient cooling of the fuel elements, the core, the reactor vessel, and the reactor cavity (including vessel supports, the cavity concrete liner, and other equipment), accomplished by a combination of conduction, convection, and thermal radiation processes. The sufficiency of heat removal is judged primarily by its prevention of failures of the barriers to fission product (FP) release. The heat removal processes during loss-of-forced circulation (LOFC) accident conditions are typically all accommodated by passive means.

Inherent reactivity control is accomplished to a great extent by the negative temperaturedependent reactivity feedback for the fuel, moderator, and reflectors throughout the entire operating (and accident) temperature range, and for the full range of the fuel burn-up cycle. Active reactivity control is provided by control rods, shutdown rods, and a reserve shutdown system. The phenomena and safety-related issues associated with these inherent and active systems are addressed in more detail in Sect. 4.

Confinement of radioactivity is provided primarily by the TRISO fuel particle coatings and fuel element graphite but also by the primary system vessels and piping, the reactor cavity confinement structures, and filters on vents from the reactor cavity and confinement, where applicable. Crucial phenomena associated with FPT are addressed in detail in Sect. 5.

Control of chemical attacks is also provided by the confinement barriers listed above in limiting the quantity of air that can ingress into the reactor core during a break in the primary system boundary. In addition, by design, sources of water ingress during normal operation are limited by maintaining a higher pressure of the primary coolant helium. However, attention must be given to the administrative controls to limit water sources that may ingress during depressurized shutdown operations such as refueling. In this study, it is assumed that there is no steam generator connected directly to the primary circuit.

\subsection{MAJOR PHENOMENA AND PROCESSES OF INTEREST}

The most significant accident $\mathrm{T} / \mathrm{F}$ phenomena rated as high importance with low or medium knowledge level are listed in Table 7 of the PIRT Main Report [3-1]. Of these, the primary phenomena of interest (i.e., the recommended primary focus for gaps in this category) are 
1. core coolant bypass flow (normal operation),

2. core effective thermal conductivity [depressurized loss-of-forced circulation (D-LOFC)],

3. reactor cavity cooling system (RCCS) performance (during LOFCs), and

4. confinement with reactor cavity air ingress (air ingress accident).

\subsection{EXPERIMENTAL AND ANALYTICAL MODELING ASPECTS OF PHENOMENA-BACKGROUND}

One of the simplifying aspects of modeling graphite-moderated gas-cooled reactor accidents is that there is no need for two-phase in-core flow models. Also, in the vast majority of severe accident cases, physical geometries do not change (i.e., there are no fuel melting or relocation phenomena as in the case of severe accidents in water-cooled and liquid-metal-cooled reactors). On the other hand, an unfortunate aspect of modeling HTGRs is that there is always a scarcity of in-core temperature and flux measurements (as compared to water-cooled reactors, which have significant in-core instrumentation) to validate the models. Thus, the reactor core thermal analyst faces uncertainties and questions about model accuracy and validity, albeit on systems that respond much more slowly (both thermally and neutronically) and typically have much greater thermal margins as compared to lightwater reactors (LWRs).

Standard core heat transfer algorithms and correlations are available for predictions of heat transfer in HTGR cores and have been used for decades in analyzing gas-cooled reactor design and operation. The NGNP, with its reliance on passive safety, will most likely use codes that include validated conduction, radiation (now with more emphasis on this mode due to the higher temperatures involved), and convection models for heat transfer.

As the vendor strategy is developed and matures, the NRC will parallel its own strategy along with its needs for confirmatory safety-related validation data. In this vein, it is recommended that as the designer/vendor prepares validation studies, the NRC should be aware of these studies as they are ongoing to ensure that the phenomena of most interest are well covered.

The following sections summarize the recommended features and potential (needed) improvements for accident codes covering $\mathrm{T} / \mathrm{F}$ and neutronic behavior, including chemical attack scenarios.

\subsubsection{T/F and Neutronic Accident Analysis Code Attributes}

Accident codes typically model the core T/F and neutronic behavior in both steady-state and transient conditions. At present, these codes normally use two-dimensional or three-dimensional $\mathrm{T} / \mathrm{F}$ models of the reactor core. The use of a multidimensional model is needed to simulate the temperature profiles and flow distributions in tall cylindrical annular cores, particularly when local or asymmetric effects are important. In pressurized loss-of-forced circulation (P-LOFC) accidents, for example, there are significant recirculation (bidirectional) flows within the core. Characterization of hot plumes exiting the top of the core depends on these flow rates. In transient analyses, point kinetics approximations are often used for the neutronics because the three-dimensional flux shape transient effects in the core during postulated accidents (other than rod ejections) are relatively small, especially when compared to those in LWRs. Multidimensional neutronic codes are necessary for providing steady-state power peaking distributions for accident codes that use point- or 1-D neutronic models. For anticipated transients without scram (ATWS) events, the dynamics of xenon-135 and samarium-149 poisoning may need to be considered. These models are sometimes also used in combination with the overall-system $\mathrm{T} / \mathrm{F}$ codes. 
The following is an annotated "checklist" covering some of the recommended features for accident simulation codes, especially noting those features needed when coupling the neutronics to multidimensional $\mathrm{T} / \mathrm{F}$ core simulations.

\section{Accident Simulation Codes_-Recommended T/F Features}

- Include dose- and temperature-dependent graphite thermal properties, noting the relatively high core effective thermal conductivity (with large uncertainties), and consideration of annealing effects, particularly in prismatic cores, during long-term heatups (especially in D-LOFC accidents).

- Incorporate effective temperature-dependent core thermal conductivity for pebble-bed reactors (PBRs).

- Model reactor pressure vessel (RPV) heat removal in LOFC events by the RCCS, where most (typically $\sim 70-90 \%$ ) of the heat transferred from the RPV to the RCCS is by thermal radiation, and the balance is by natural convection of the reactor cavity air.

- Consider natural circulation (air) heat transport in the reactor cavity. Even though most of the heat is transferred to the RCCS by thermal radiation, the distribution of heat and temperatures within the cavity (especially at the cavity ceiling adjacent to RPV penetrations for control rods) is affected by convection terms.

- The RCCS has important safety functions within the design bases and, therefore, should be carefully modeled and coupled to the RPV and core T/F models (refer to NRC General Design Criteria for residual heat removal, emergency core cooling, and containment cooling).

- Include maximum fuel temperature plus time-at-temperature calculations (two critical limiting factors) for all fuel regions, providing inputs to fuel failure models to estimate source terms.

- Consider core pressure drop correlations (particularly for the pebble bed)—standardized and well-documented correlations are available, at least for operating flow regimes, showing that the pressure drop is very sensitive to assumed packing fractions of the pebbles.

- Consider core heat transfer algorithms - correlations are generally available for the prediction of heat transfer in HTGR cores, and these include conduction, radiation, and convection, both at normal (operating) and LOFC conditions.

- Model the changes of flow (and flow direction) within the core, including recirculation within the core in P-LOFC accidents.

- The modular HTGR core is relatively large, so from the fuel zones to the RPV there are large temperature gradients. There are also large thermal gradients in the lower reflector and core support blocks due to nonuniformities in radial power density profiles, coolant flows, and coolant temperatures. Likewise there are many other coolant flow paths within the RPV, with significant flows typically bypassing the fuel regions. The amount of bypass flow is difficult to estimate due to variations in gap sizes (due to irregularities, thermal gradients, and irradiation-induced deformations). Bypass flows can have significant effects on maximum fuel temperatures during operation. For fast transients, especially, detailed temperature profiles of the fuel and graphite should be taken into account for thermal stress calculations. 


\section{Accident Simulation Codes-Recommended Neutronic features}

- The afterheat (total and spatial distribution) as a function of time after shutdown is a critical parameter in calculating maximum fuel temperatures during LOFC accidents. Typically, afterheat correlations rely mostly on calculations for the long-term estimates.

- Negative temperature-reactivity feedback coefficients for the fuel, moderator, and reflectors are temperature and burn-up dependent, with potentially positive coefficients for the heat-up of the central reflector.

- Neutronic codes need to account for core heterogeneity. To obtain accurate resonance self-shielding of the cross sections, it may be necessary to model the detailed heterogeneity of the fuel. For example, in pebble beds, the fuel particles should not be smeared into the graphite matrix, and there should be a capability for handling cross sections dependent on temperature.

- The code should account for the time dependence of xenon-135 and samarium-149 poisoning effects for ATWS cases.

- The analysis should account for the effect of asymmetrical control rod movements and xenon-135 oscillations.

- While significant reactivity insertion accident (RIA) events are not considered to be likely in current modular HTGR concepts, they cannot be ruled out entirely until the designs are finalized. The neutronic codes may be needed to model these types of events.

- In the case of steam (direct) cycle designs, the effects of water ingress on reactivity and control rod worth need to be included, since potentially large amounts of steam or water could enter the core during power operation.

\subsubsection{Whole-Plant Modeling Codes}

There are several important design features related to the whole-plant transient $\mathrm{T} / \mathrm{F}$ behavior of modular HTGRs that should be modeled.

- The coupled dynamic helium flow and heat transfer in the core, the turbine(s) and compressor(s), the recuperator, the helium/water heat exchangers (or steam-generator system) balance of plant (BOP), and/or the helium circulator and associated intermediate heat exchanger (IHX) supplying the chemical processing (or other) plants. A model of the shutdown cooling system (SCS) is also required.

- To model the primary circuit with the BOP, the code should be capable of simulating a one-dimensional helium flow network, including the capability to model changes in flow direction in some accidents.

- For accident studies involving a complete loss of flow through the BOP, the BOP dynamic response can usually be ignored, except for estimating system pressure changes in the P-LOFC accidents.

\subsubsection{Confinement Analysis Codes}

Confinement analysis codes are needed to calculate pressure, temperature, and gas composition transients in the reactor-cavity confinement space(s) during depressurization accidents involving eventual air ingress into the RPV and core. If subdivided, the confinement compartments and the paths between compartments are typically modeled by nodes with given volumes and junction characteristics. Gas compositions calculated in the compartment(s) account for air, helium, products of oxidation, and steam (for water-ingress accidents). Gas temperatures and compositions in the vicinity of the primary system breach are used to determine subsequent ingress flows into the reactor 
core. Considerations are made for air in-leakage to confinement space(s) and for any special injections of inert gas or flow retardants (by the operators) during recovery from a long-term accident.

For cases assuming damage caused by a rapid depressurization, changes in compartment or junction characteristics or relief valve sealing capability would need to be modeled. A large pressure pulse could damage the RCCS, reducing cooling capabilities and/or opening up another release path both for air/water ingress into the reactor cavity, as well as for possible FPT out to the environment.

There are significant differences between loss-of-coolant accidents (LOCAs) in a largely steam atmosphere in LWRs and a largely inert gas atmosphere in HTGRs. One difference is due to the lack of a phase change (condensation) in cooling high-temperature helium as compared to steam, thus increasing the importance of gas heating and cooling effects from direct contact of the helium with the confinement structures. As a result, a depressurization accident in the case of a sealed ("airtight") containment could retain the high pressure for a very long time, thus providing a significant driving force for subsequent discharges of the entrapped gas, which may become more contaminated as the accident progresses. This potential accident sequence is a major factor in the (much-preferred) design selection of a vented confinement over a sealed containment building.

Aerosol and dust generation and transport would also be different if the confinement system was dry rather than wet, and building filters would have to operate (and survive) at high temperatures. Organic forms of iodine can challenge filter designers when confronted with varying conditions. Aerosol and dust issues are covered in detail in Sect. 5.2.3.8.

\subsubsection{Codes Dealing with Chemical Reactions}

Special code features are required to simulate chemical reactions due to air and possibly for water/steam ingress into the core. These models need to work in conjunction with $\mathrm{T} / \mathrm{F}$ codes and (for the water ingress) with codes that simulate the neutronics, due to possible steam/water effects on reactivity for ATWS cases. Chemical reactions may also result in additional releases from damaged fuel, or failure of fuel damaged by a temperature transient, or in possible release of FPs absorbed in the graphite structures.

In air ingress accident scenarios, it is typically assumed that a D-LOFC is followed by ingress of ambient air or mixed gasses from the reactor-cavity confinement space into the primary system at the point of the break, with eventual transport through the primary system to the core. This may occur either just after the depressurization is complete (to ambient pressure) or later. The oxidation of core graphite that follows generates heat (and $\mathrm{CO}_{2}$ ), in addition to the afterheat, and the gas flows upward in the core and can subsequently provide some convective cooling (or heating). $\mathrm{CO}_{2}$ entering the mid-to-upper part of the core is likely to encounter higher temperatures that cause an endothermic (Boudouard) reaction with core graphite and produces CO. Large-scale oxidation of the core graphite could cause structural damage and expose fuel to chemical attack. The change in the reactor atmosphere from reducing to oxidizing may affect the plate-out of FPs onto the metal components. These FPs could then be entrained in the convective flow and eventually exit the RPV.

Graphite oxidation models rely on data for specific types of graphite and their irradiation histories. For lower temperature ranges, the oxidation rates are determined by the chemically controlled rate, while at higher temperatures, the process is usually mass-transfer limited. These important distinctions must be included in the modeling. Oxidation of graphite at lower temperatures is more likely to cause structural damage.

Key factors are the net airflow rate into the RPV and core area, and ultimately the "availability" of fresh air (oxygen) over the course of the accident. The net airflow through the core is strongly dependent on the buoyancy forces due to differential temperatures and composition and to the flow resistances in the core and at the break(s). Other scenarios that involve forced air convection (such as 
by operation of the SCS circulator) have the potential to cause much more damage, or at least to increase oxidation rates until the available oxygen is depleted.

For a single break or opening in the primary system, calculations and confirmatory experiments have shown that it may take a long time (days) before a significant natural circulation net air inflow into the core is established. This process involves molecular diffusion of air, eventually into the helium-filled top region of the reactor vessel. For a much less likely case of a double break in the vessel that allows ambient air access to both the top and bottom of the core, a chimney-like configuration would promote a higher net ingress flow that would be established more quickly than in the case of a single break.

Experiments have shown that $\mathrm{SiC}$ TRISO fuel is resistant to failures from exposure to air at temperatures below $\sim 1300-1400^{\circ} \mathrm{C}$ [3-2]. In typical air ingress accident scenarios following a singlebreak D-LOFC, calculations show that oxidation is typically limited to the lower areas of the core where the temperatures are much less than $\sim 1300^{\circ} \mathrm{C}$, so FP releases even in severe ingress sequences may be limited.

Water and steam ingress modeling has many similarities to air ingress, as both have corrosive effects on the graphite and can cause structural and fuel damage. Water/steam reactions at high temperatures are endothermic, however, rather than exothermic. Modular HTGR cores are typically under-moderated, so steam/water mixtures entering the core are likely to increase reactivity, which could then cause significant power increases if the accident also assumes an ATWS, with subsequent recriticality of the core, so fuel damage could occur with potentially significant power surges.

Since the water-cooled heat exchangers in direct-cycle gas-turbine designs typically keep waterside pressures lower than the primary helium pressure (except during shutdown), water/steam ingress accidents are usually only of concern for the (direct) steam-cycle plants, where steam pressures are much higher. However, water ingress is not impossible just because the cooling water pressure is normally lower than the reactor operating pressure. Water could still enter the high-pressure core through pump action, or if the break allows the water source to pressurize in a vertical configuration (water supported by gas), in which case instabilities could drive the water into the core.

Due to the wide range of uncertainties in terms of possible ingress rates and total ingress quantities for both air and steam/water cases, parametric (scoping) studies are recommended.

In summary, for codes that model chemical attack, consideration should be given to

- chemical reactions between graphite and air/steam;

- coupling between T/F codes and chemical kinetics models;

- formation of gases and aerosols containing FPs;

- details of the confinement holdup volumes, flow paths, and stratification;

- graphite FP inventories prior to the accident;

- reactions between the released FPs and the oxidizing nature of the gas flow;

- local fuel temperatures and the potential for an aggressive chemical environment which may increase fuel failures and failed fuel releases of FPs; and

- data available on TRISO fuel failure in oxidizing atmospheres.

\subsection{CLOSING THE GAPS-STAGES OF MODEL DEVELOPMENT}

The following areas have been identified as those in which gaps remain between what is needed for sufficient safety analysis vs what is generally available in the form of operationally validated 
models with adequate supporting data. Reductions in the uncertainties in these models and data generally result in a reduction of the margins required to make an adequate safety case.

\subsubsection{Near-Term Approaches}

\section{Core-Coolant Bypass Flow Phenomena (Normal Operation)}

The modular HTGR core is relatively large for its power level and operates at very high temperatures. Thus from the fuel zone (radially) to the RPV there are large temperature gradients. Likewise there are many coolant flow paths within the RPV, with significant flows that can bypass the fuel regions (a power-to-flow ratio concern). The amount of bypass-flow is difficult to estimate because of variations in gap sizes (due to thermal gradients, manufacturing, and irradiation-induced deformations and gaps). Bypass-flow can have a significant effect on maximum fuel temperatures during operation.

If core coolant bypass-flows and channel bypass-flow are to be better characterized, an overall understanding of the specific design aspects that affect this phenomenon is needed. This may include a more complete understanding and accounting of block bowing for the graphite used for fuel [for prismatic-core modular reactors (PMRs)] and reflectors in the core barrel configuration. Some instrumented testing of models of stacked blocks could be undertaken to better understand the phenomena. Large temperature redistribution transients were seen at the Fort St. Vrain reactor due to shifting columns of fuel blocks; however, the configuration in the Fort St. Vrain core was different from current PMR designs since a flow-control orifice over each set of seven columns of fuel was used, and each column of fuel blocks was shorter than in current designs.

One proposed NGNP PMR fuel block design has an overlapping, around-the-edge lip of graphite on the bottom of the block and chamfered edges on the top of the block that would provide a radial flow-retarding interlock, in addition to the dowel pins and holes used to provide inter-block connections for axial stability. Without in-core testing, it may be difficult to know how these differences would impact column stability.

Given the existing $\mathrm{T} / \mathrm{F}$ codes, it is possible to perform a parametric analysis on gap configurations (with assumed tolerances and worst cases defined). This may help bound the questions associated with gap and bypass flows and the phenomena of shifting blocks.

\section{Effective Core Thermal Conductivity}

For prismatic cores, it is suggested that dose and temperature-dependent graphite thermal properties (especially thermal conductivity) be made available to the NRC T/F code suite. This is to account for large uncertainties, as well as for characterization of annealing effects during long-term heat-up D-LOFC accidents.

There are also considerable error bounds in effective core thermal conductivity (as a function of both temperature and irradiation) for pebble bed cores. The correlations available are empirical. The Pebble-Bed Modular Reactor (PBMR) Pty. Ltd. project has an experimental facility to be used to refine the database.

\section{Afterheat Correlations}

Peak fuel temperatures in the D-LOFC accident are very sensitive to the afterheat (vs time) to the same extent as they are to the core thermal conductivity function. Afterheat correlations are sensitive to fuel type and burn-up histories; however, tracking fuel histories during operation can be challenging. In addition, afterheat validation data is more difficult to obtain for long times after shutdown. 


\section{Core Effective Pressure Drop}

For the PBR, standardized and well-documented correlations for core pressure drop are available, but more conformation data may be needed for low-flow cases to better characterize flow distribution and plume formation (for the P-LOFC) and in-core airflow distributions during air ingress accidents. Data show that the pressure drop is very sensitive to assumed packing fractions of the pebbles. So, here again, parametric analyses using established ranges of different packing fractions can help define a performance envelope. The PBMR Pty. Ltd. project has an experimental facility to be used to refine pebble-bed core pressure drop correlations, but (perhaps) only at the higher flow conditions.

\section{RCCS Performance (During LOFC)}

The RCCS has important safety functions and needs to be simulated in detail, with its predominantly radiant heat transfer coupling to the RPV and other heat transfer mechanisms within the reactor cavity. The RCCS functions include maintaining the reactor cavity liner concrete temperature below prescribed limits (typically less than $100^{\circ} \mathrm{C}$ ), preventing the RPV peak temperature from exceeding limits during LOFC events, and minimizing parasitic heat losses during normal operation. While most of the heat transferred from the high-temperature RPV to the RCCS is via $\mathrm{T}^{4}$ radiation, air convection within the cavity has a significant effect redistributing the heat.

Models may be needed to simulate large pressure pulses in D-LOFC accidents that could damage the RCCS, reducing cooling and/or opening up another release path for air or water ingress to the reactor cavity and perhaps FPT out to the environment.

RCCS operation and previous comparison/validation efforts for the RCCS models have historically been shown to be problematic and not nearly as straightforward as expected [3-3]. There are international research opportunities that should be taken advantage of with Japan, and perhaps China and South Africa, to ensure that RCCS designs can be modeled sufficiently. Japan [hightemperature engineering test reactor (HTTR)] and China [high-temperature reactor, $10 \mathrm{MW}$ (HTR10)] have RCCS operating data that could be useful for model verification and validation (V\&V) as well as practical operating experience that could be useful for design efforts. These opportunities should be pursued when available, even though the basic design features of the NGNP RCCS (e.g., air or water cooled) have not yet been established.

\section{Fuel Performance Models}

Aspects of maximum fuel temperature plus time-at-temperature histories (critical limiting factors) for all fuel regions provide inputs to fuel failure models to determine source terms and dose-vsfrequency estimates. This is an important cross-cutting aspect. Chemical reactions in air (or water) ingress accidents which depend on temperature are also a cross-cutting item that should be included in the T/F codes. See Sect. 5.2.3.1 for more details. For fast transients, especially, detailed temperature profiles of the fuel and graphite should be taken into account for thermal stress calculations.

\section{Air Ingress Phenomena}

Air ingress accident scenarios are difficult to characterize because even with a well-established confinement design, there would be an extremely wide variety of possible accident sequence boundary conditions (leak size, location, operator action, etc.). At this point, with little or no detail available about the confinement, only generalized studies and experiments would be practical. Bounding analytical studies, however, could be useful in determining positive (and negative) features of proposed design characteristics. The major features of general interest would be quantification of long-term air in-leakage into the confinement and the mixing and stratification characteristics of gases in prototypical cavities within the confinement. 


\subsubsection{Intermediate- and Long-Term Approaches}

There are wide varieties of features and capabilities of the accident analysis codes currently used for modular HTGRs. The range of capabilities, as well as the V\&V of these codes, is important in determining how effectively they can be used in assessments of postulated accidents and safety cases. This is especially true now because of the lack of more detailed reactor designs to evaluate and the deficiencies in the supporting databases.

Over the long term, addressing these gaps will evolve in parallel with the development of better definition and more detail in the eventual NGNP design. The long-term goal should be to have a comprehensive suite of verified and validated analysis codes, agreed-upon accident cases for regulatory acceptance, and robust supporting databases that NRC can use for independent confirmatory analysis.

Until more design details are available, significant uncertainty will remain for the types of accidents to be reviewed and the analytic approaches to be used. Sensitivity studies can play an important role in the eventual determination of an R\&D program. Some general guidance for the development, V\&V of accident analysis codes is provided in Sect. 6 of International Atomic Energy Agency (IAEA) Safety Reports Series (SRS) No. 23 [3-4] and another IAEA SRS report specific to modular HTGR accident analysis just released, SRS No. 54 [3-5]. Also, a new American Nuclear Society (ANS) safety standard (ANS 53.1) dealing with accident analysis computer codes is currently being developed.

\subsubsection{Summary Descriptions and Evaluations of Selected Existing Code Capabilities}

Limited information is available on the status of many of the world's major HTGR accident codes in use, especially those being developed for the large programs now building modular HTGRs. China and the Republic of South Africa (RSA) are developing and manufacturing major systems and components for complete PBR designs. Major test facilities are being employed where the technologies, including software development, are progressing rapidly.

PBR simulators are being developed in China by the Institute of Nuclear Energy Technology (INET) at Tsinghua University in Beijing and include 3-D core $\mathrm{T} / \mathrm{F}$ and overall system calculations. Their code suite HTRSIM is based on the standard German pebble-bed codes THERMIX and TINTE [3-6]. TINTE is a well-established reactor dynamics code with 2-D neutronics and thermal-fluid capabilities for primary loop modeling. A block diagram of the TINTE code, as adapted by INET, is shown in Fig. 2. Water and air ingress modeling has been incorporated. INET also uses KORIGEN for FP inventory calculations, FRESCO 2 for FP release, and LOOPHTR for primary loop radioactivity inventory calculations. 


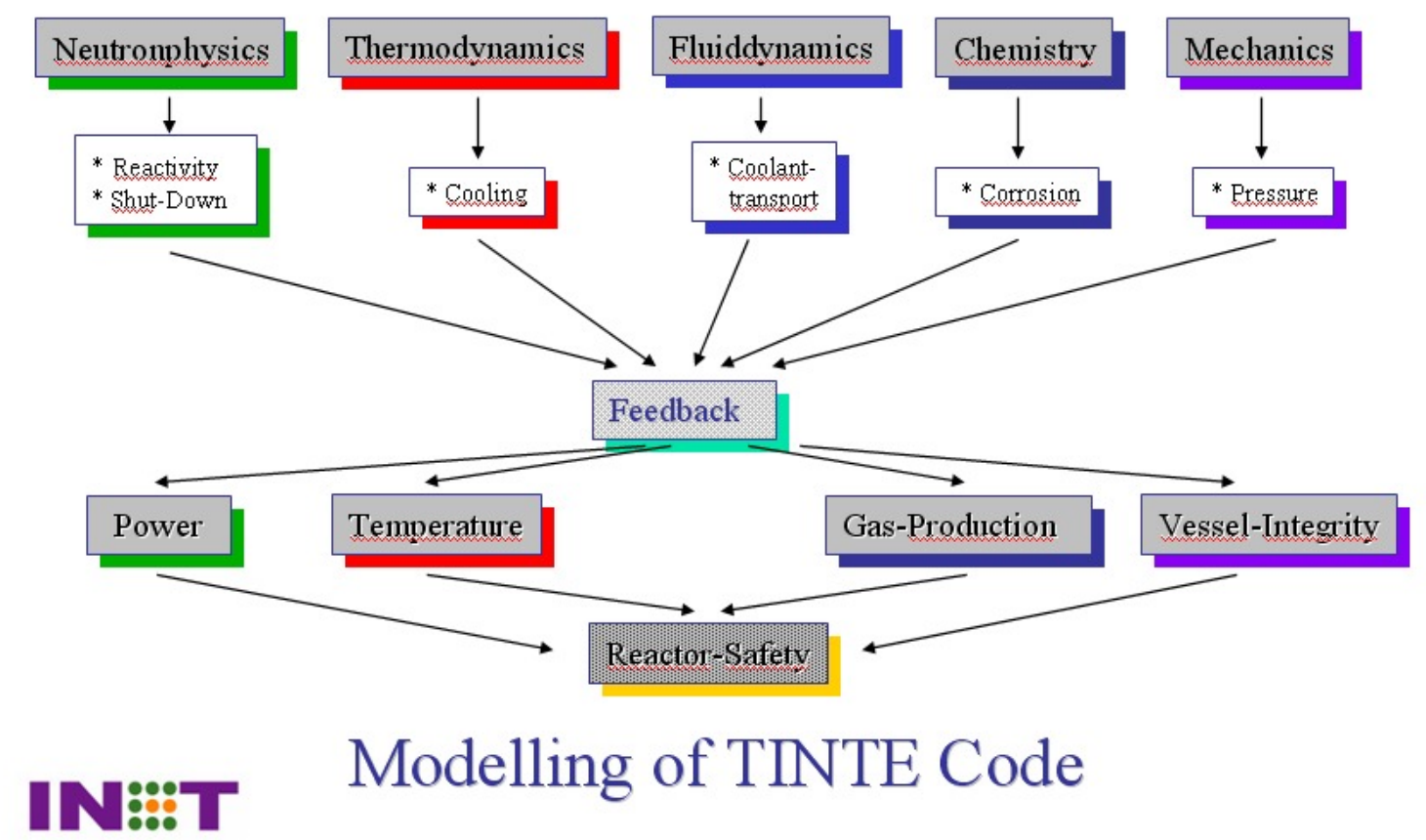

Fig. 2. TINTE code modeling block diagram as developed by INET.

The RSA accident analysis software is being developed using Flownet Nuclear, a commercial simulation package that has been adapted for PBMR accident (and operational) calculations for all accident sequences noted except for the air ingress accidents (under development). The project also uses the German TINTE code for pebble-bed core accident calculations. The FLUENT computational fluid dynamics (CFD) code is used for detailed fluid-flow and heat transfer calculations. The ASTEC code, jointly developed by L'Institut de radioprotection et de sûreté nucléaire (IRSN, France) and Gesellschaft für Anlagen und Reaktorsicherheit mbH (GRS, Germany) for LWR severe accidents, is incorporated into the code suite to model FPT, aerosols, and containment/confinement $\mathrm{T} / \mathrm{F}$ behavior.

The South Korean program at the Korean Atomic Energy Research Institute (KAERI), which began in 2004, has a very comprehensive accident code system for that country's HTGR design, which is dedicated to hydrogen production-MARS-GCR for system design and safety analysis and GAMMA (Gas Mulit-component Mixture Analysis) for air ingress events. A recent development of the GAMMA+ code suite includes coupling with neutronics (CAPP) and SANA for gas turbine modeling. Dust, FP, and tritium modeling is combined in the MELCOR and PADLOC codes. MIDAS is used for FPT analysis and LILAC for detailed computational fluid dynamics (CFD) analysis. KAERI codes are adapted to both prismatic and pebble-bed core designs.

The Japanese code ACCORD is typically used for comprehensive reactor accident simulations (except air ingress). It employs modules for nuclear calculations, heat transfer calculation of the reactor core, heat exchangers and piping, and flow calculations for the primary and secondary coolants. The control system and safety protection system for the HTTR are incorporated in this code. Reactor power is computed using a point kinetics approximation model with six delayed neutron groups. The reactor core is simulated by a one-channel model with one representative fuel rod. Each heat exchanger is simulated by a one-channel model with one (average) heat transfer tube.

The TAC-NC code is also used as a two-dimensional and time-dependent thermal analysis code for safety evaluations of the large JAEA (JAERI) HTGR designs and has been applied to benchmark calculations of the 10-MW(t) high-temperature (HTR-10, China) using a R-Z cylindrical calculation 
model. The region of the calculation model is from the center of the reactor core to the water-cooling panel outside the insulating plates of reactor cavity for the radial direction, and from the top air cavity to the bottom air cavity outside the RPV for the axial direction. TAC-NC code improvements were made to consider ATWS calculations. A point kinetics model with six delayed neutron groups with xenon poisoning is used. For PBRs, the reactor core is modified as a smeared model including pebble-bed balls and helium coolant.

Russian codes GTAS-M and DUPT (by OKBM) are the major ones used for accident simulations. GTAS calculates core thermal hydraulics in 2-D (R-Z geometry) using energy equations for porous media. Flow equations are solved using quasi-stationary 1-D approximations for each channel. GTAS is used for D-LOFC and P-LOFC accidents as well. Heat transfer in the reactor cavity due to radiation and convection is calculated by a simplified model. The GTMHR code platform [3-7] is used for analysis of transients, and its mathematical models encompass all major reactor plant elements, including point kinetics with a reactivity control system and heat transfer in the core with fuel assemblies made up of hexahedral blocks. Models of the turbomachine with associated heat exchangers, valves, and control systems are included as well. The major models have been verified by comparing analysis results with experiments and other analytical solutions.

Russian calculations of helium-air mixtures in the primary circuit and in the containment or confinement vault after depressurization are done using the RPK and MACEX codes. The RPK code calculates helium outflow parameters during depressurization, as well as helium-air mixture parameters in the primary circuit and containment, accounting for gas displacement afterwards with temperature variation and vault air leakages. The MACEX code is used to calculate helium-air convective and diffusion mass transfer through tubes. The code implements dynamic equations for 2-D concentration diffusion, momentum, continuity, and gas composition. Code verification exercises used experiments that were performed at specially created test facilities [3-8]. GTAS code verification also made use of benchmarking exercises in an IAEA coordinated research projectCRP-3 [3-3].

In The Netherlands, the Nuclear Research Group (NRG) has developed the PANTHERMIX code, which combines THERMIX-DIREKT (from FZJ), a 2-D (R-Z) HTR thermal fluids accident code for PBRs, with PANTHER models developed at NRG that can incorporate 3-D neutronics. RELAP-5 is also incorporated for full-system simulations. Also under development is the SPECTRA code, which will incorporate dust contamination estimation.

The AREVA program (U.S.) has developed a comprehensive HTGR accident simulator for ANTARES [3-9] and uses the MANTA code for more detailed core 3-D design issues.

For accident analyses, the coupled thermal hydraulic and chemical reactions code REACT/THERMIX is applied. The coupled code consists of three modules-THERMIX, KONVEK, and REACT. The THERMIX module is a general purpose steady-state or transient twodimensional heat conduction module that calculates temperatures in the structures. The twodimensional quasi steady-state convection module KONVEK calculates the temperature and flow distribution of the fluid. The two-dimensional quasi steady-state reaction module REACT calculates gas concentrations, reaction heat, and graphite burn-off in the case of air ingress accidents. In general, the calculation employ two-dimensional symmetric models in ( $r, z)$ geometry, covering the whole reactor in its cavity, including the RCCS.

Other U.S. codes in common use are PEBBED [3-10] [Idaho National Laboratory (INL)] and GRSAC [3-11] [Oak Ridge National Laboratory (ORNL)].

PEBBED is a 3-D core simulator developed at INL specifically for PBR design and analysis. It converges directly upon the asymptotic or equilibrium fuel cycle using an integrated neutron diffusion-depletion solver. Core design optimization is performed using a genetic algorithm 
operating on core geometry and pebble flow parameters. Core temperature profiles are obtained using a one-dimensional embedded T/F solver or with coupled THERMIX or RELAP calculations. THERMIX is a 2-D (R-Z) heat transfer and gas dynamics code developed for the German PBR program. It is part of the Very Superior Old Program (VSOP) PBR fuel cycle analysis system [3-12]; however, the THERMIX module has been extracted recently for use with many other codes. THERMIX contains material properties and correlations validated for PBR analysis under the German programs. PBMR (Pty. Ltd.) uses a modified version of VSOP with THERMIX for their reactor design and fuel cycle analyses [3-13].

The Graphite Reactor Severe Accident Code (GRSAC) development, use, and validation exercises began over 30 years ago at ORNL with several predecessor codes. Current interest in GRSAC involves the development of confinement models for air ingress accident modeling, simulation of accident scenarios for modular HTGR designs, and simulation of benchmark transients run on the HTTR (Japan) and HTR-10 (China). GRSAC employs a fairly detailed ( 3000 nodes) 3-D thermal-hydraulics model for the core, plus models for the reactor vessel, SCS, and RCCS. There are options to include ATWS accidents using a point kinetics model and to model air ingress accidents, simulating the oxidation of core graphite.

In general, all these codes typically use the universally available data for all aspects of the simulation but with widely varying levels of detail and simulation methodologies. Brief descriptions of the codes used by IAEA Coordinated Research Project (CRP) participants can be found in TECDOC reports of CRP-3 and CRP-5 [3-3, 3-14]. A more detailed description and tabulation of modular HTGR accident codes is in an NRC letter report (in publication) [3-15].

\subsection{SUMMARY}

The primary areas in accident $\mathrm{T} / \mathrm{F}$ analysis where there are significant gaps between what is needed vs what is available have been identified as

1. core coolant bypass flow (normal operation),

2. core effective thermal conductivity (D-LOFC),

3. RCCS performance (during LOFC), and

4. confinement with reactor cavity air ingress (air ingress accident).

The extent of the concern and the need for more R\&D in these areas depend on the eventual NGNP design, including the extent of built-in safety margins. It is likely that all NGNP designers are cognizant of these concerns and are factoring in mitigating attributes.

In the PIRT main report section on accident and T/F analysis [3-1], a number of phenomena were identified in the "normal operation" category as high importance and low knowledge [H, L], namely, core bypass flows, core flux/power profiles, temperature-reactivity feedback functions, outlet plenum flow distribution, fuel performance, and silver $(\mathrm{Ag}-110 \mathrm{~m})$ release. Of these, only the first (bypass flows) was identified in this gap report as needing special regulatory attention. This is because the core flux/power profiles and temperature-reactivity feedback functions, while important, can normally be calculated with sufficient accuracy to ensure safe operation, and in some cases confirmed by measurement or test. These are among the many features of modular HTGRs that require allowances for considerable margins for error to ensure operating conditions do not adversely affect plant systems and components. Core outlet plenum flow distribution phenomena, while important to considerations such as temperature gradients and fluctuations seen by equipment downstream (e.g., the gas turbine), are not considered to be a safety concern. Silver release (and plateout) is a maintenance dose concern, particularly for direct-cycle gas turbine designs. 
In the LOFC categories, only RCCS performance features were rated in the PIRT as critical $[H, L]$. These were, specifically, emissivities of the RCCS panels and RPV outside surfaces and the convection and heat transfer characteristics of circulating air in the reactor cavity. While these parameters would affect RPV and vessel support peak temperatures, they would have only minimal effect on peak fuel temperatures. This report recommended special attention be given to effective core conductivity and afterheat functions (in D-LOFC accidents) since peak fuel temperatures are very sensitive to these values.

In reactivity (ATWS) and air ingress accidents, there was corresponding emphasis put on crucial parameters (temperature-reactivity feedback functions and gas ingress rate and oxygen availability, respectively) in the two reports.

As a follow-on to a gap analysis, existing codes should be evaluated on the basis of how well they model the key processes and incorporate the needed attributes into a single package. For example, accident $\mathrm{T} / \mathrm{F}$ analysis codes should ideally be linked with fuel performance and FPT models to obtain estimates of the source term, enabling the analyst to obtain a clear figure of merit. Such linkages would enable parametric (sensitivity) analyses to be run with predicted source terms generated, providing quantitative risk-informed research guidance.

The long-term goal here is to have a comprehensive suite of verified and validated analysis codes, agreed-to postulated accident cases for regulatory acceptance, and a robust supporting database that $\mathrm{NRC}$ can use for independent analysis of candidate plant and confinement designs and options.

\section{REFERENCES}

3-1. S. J. Ball and S. E. Fisher, Next Generation Nuclear Plant Phenomena Identification and Ranking Tables (PIRTs)_Volume 1: Main Report, NUREG/CR-6944, Vol. 1 (ORNL/TM2007/147, Vol. 1), Oak Ridge National Laboratory, March 2008 (more details on the ACTH PIRT exercises are found in Vol. 2).

3-2. IAEA, "Fuel Performance and Fission Product Behaviour in Gas Cooled Reactors," Section 5.4, IAEA-TECDOC-978 (for CRP-2), Vienna, 1997.

3-3. IAEA, Heat Transport and Afterheat Removal for Gas Cooled Reactors Under Accident Conditions, IAEA-TECDOC-1163 (CRP-3), January 2001.

3-4. IAEA, Accident Analysis for Nuclear Power Plants, IAEA Safety Reports Series No. 23, Vienna, 2002.

3-5. IAEA, Accident Analysis for Nuclear Power Plants with Modular High Temperature Gas Cooled Reactors, IAEA Safety Reports Series No. 54, Vienna, April 2008.

3-6. U. E. Sikik, "TINTE Analysis of PBMR Reactivity Insertion Transients,” 3rd Intl. Topical Meeting on HTR Technology (paper C-72), Johannesburg, October 1-4, 2006.

3-7. V. A. Bolnov et al., "GTMHR Code Description and Potential," Proc. 2nd Int'l. topical Meeting on HTR Technology, Paper No. C27, Beijing, Sept. 22-24, 2004.

3-8. $\quad$ N. Kuzavkov et al., "Study of Gas Exchange Between HTGR Primary Circuit and Surroundings," Proc. 8th Intl. Topical Meeting on Nuclear Reactor Thermal Hydraulics, Kyoto, Japan, 1997.

3-9. H. Haque et al., "Thermal Response of A High-Temperature Reactor During Passive Cooldown Under Pressurized and Depressurized Conditions," 2nd Intl. Topical Meeting on HTR Technology (Paper F-02), Beijing, September 22-24, 2004. 
3-10. H. D. Gougar et al., "Reactor Pressure Vessel Temperature Analysis of Candidate VHTR Designs," Proc. HTR-2006, 3rd Intl. Meeting on HTR Technology (Paper C-069), Johannesburg, October 1-4, 2006.

3-11. S. J. Ball and D. J. Nypaver, GRSAC Users Manual, ORNL-TM-13697, Oak Ridge National Laboratory, Oak Ridge, TN, February 1999.

3-12. E. Teuchert et al., V.S.O.P-94, Computer Code System for Reactor Physics and Fuel Cycle Simulation Input Manual and Comments, FZJ GmbH, JuL-2897, April 1994.

3-13. F. Reitsma et al., An Overview of the FZJ Tools for HTR Core Design and Reactor Dynamics, The Past, Present, and Future, M\&C 2005, Palais des Papes, Avignon, France, September 12-15, 2005, on CD-ROM, ANS, LaGrange Park, IL (2005).

3-14. IAEA, Evaluation of HTGR Performance, IAEA-TECDOC-1382 (CRP-5-Part 1), Vienna November 2003. (The TECDOC for Part 2 is in publication.)

3-15. S. J. Ball and R. N. Morris, Comprehensive Survey of HTGR Design and Safety Analysis Tools for NGNP, ORNL/NRC/LTR-07/07, Oak Ridge National Laboratory (in publication). 


\section{REACTOR PHYSICS}

While there are some gaps in reactor physics experimental data, as discussed further below, there are no significant gaps in reactor physics analysis capabilities and databases currently available in vendor codes to model the neutronics aspects of the proposed NGNP concepts. However, special attention should be given to the following.

In preparing multigroup or few-group cross sections for discrete-ordinates transport theory analyses or diffusion theory analyses, the cross section processing codes should be able to incorporate properly the effect of double and possibly triple heterogeneities in calculating the resonance integrals for the coated particle fuel. The capability to calculate resonance integrals accounting for the neutron flux depression due to the overlap of other resonance absorbers in the fuel is also important. In preparing few-group cross sections in the thermal energy spectrum, cross section processing codes should also account for the resonance broadening in isotopes with thermal resonances and the selfshielding effects of double or triple heterogeneities due to the spatial depressions in the thermalneutron energy spectra. Monte Carlo codes using continuous-energy cross sections should be able to handle the probability distributions associated with randomly distributed coated fuel particles in the fuel compacts so that double and/or triple heterogeneities are addressed.

Since the stability of cores to xenon-135 oscillations can be assessed more readily if the eigenvalues and flux eigenfunctions of the first and second harmonics can be calculated using diffusion theory [4-1], the capability is needed to calculate these higher order harmonics. The difference in the fundamental mode and first harmonic eigenvalues can be used to evaluate the socalled $\lambda$-mode stability and, thereby, used to judge the susceptibility of the core to either axial or azimuthal xenon-135-induced oscillations for the burn-up and for controlling critical rod positions as a function of core life [4-2]. The use of these harmonic flux distribution results in the axial or azimuthal dimensions would be useful for quasi-static analyses of reactor kinetics calculations of the slower xenon-135 transient, with control rod motion and thermal feedback effects included, or in more simplified two-node point kinetics models of the core with node-dependent feedbacks.

As noted above, there are some gaps in the experimental data such as adequate measurements of the core operating temperature and the combined reactivity effects of high temperature and the reactivity contributions of FPs such as xenon-135. Fissionable production products such as plutonium-239 and plutonium-240 also affect the temperature-dependent coefficients of reactivity. As discussed in detail in this section, good data on these effects are both hard to measure and have typically not been measured with sufficient defensible rigor or relevance in past experiments or tests. Near in-core (temporary) measurements may be feasible in PMR designs utilizing inner reflector control rod penetrations. Temporary flux, pressure drop, and temperature measurements were made in the Fort St. Vrain reactor utilizing an instrumentation package that was substituted for a control rod. Attention should be paid to current testing in the HTR-10 and HTTR and to the development of high-temperature sensors and neutron detectors that can be used in the first unit of the NGNP. Without adequate instrumentation there will be limited test data, and the interpretation of start-up physics test results will end up being highly dependent on calculated, as opposed to measured, data. This has been the case for many past HTGRs, Documentation of experiment-to-calculation analyses of key reactor physics parameters is important in demonstrating the safety margins of the reactor [see Sect. 4.3 of Ref. 4-3 and Appendix A, respectively, in Refs. 4-4 and 4-5]. 


\subsection{MAJOR PHENOMENA OF INTEREST}

\subsubsection{Background in Selection of Phenomena}

In both the fuel PIRT [4-6] and the current PIRT [4-7], several accidents were considered that were based on assumed reactivity-driven or power-distribution-affected scenarios. The fuel PIRT considered the following two accidents of this type:

- reactivity insertion based on the effect of rod ejection in the PMR, given excess reactivities representative of that in a PBMR but applied to conditions in the PBR, combining reactivity and power-distribution effects; and

- power pulse of several seconds in duration, a reactivity-driven effect.

The current T/F-accident PIRT considered the "challenge to reactivity control" as one of the groupings for phenomena based on challenges to fundamental safety functions. As background during the PIRT panel deliberations, and as discussed in Sect. 4.1.2 below, the PIRT panel considered both events at the Fort St. Vrain reactor that impacted the operability of reactivity control systems, and results from the NRC preapplication review of the DOE modular high-temperature gascooled reactor (MHTGR) [4-3] that related to the potential recriticality during an extended loss-ofcooling event. In addition to the accident scenarios identified in the fuel PIRT, there were three additional scenarios of this type that were considered in the current PIRT based on past plant operating experience:

- restart and operation of the reactor following an undetected major water ingress during shutdown, a reactivity-driven effect;

- restart and operation of the PMR following refueling with an incorrect positioning of fresh fuel such as reverse loading, which should be observable in the expected critical position of control rods and impact on peak fuel temperature during operation, combining reactivity and power-distribution effects; and

- impact of control rod misalignment on power peaking and initiation of azimuthal or axial xenon oscillations with subsequent power peaking, and the subsequent impacts on peak fuel temperatures during operation, combining reactivity and power-distribution effects.

The PIRT tables in the main report list and provide the expert opinion on specific phenomena of interest. Those with a high-to-medium importance rating with a low-to-medium knowledge level are listed as follows:

- the time-dependence and spatial distribution of decay heat as a major factor in determining the maximum fuel temperature during a D-LOFC [importance- - high; knowledge level — medium, or $(\mathrm{H}, \mathrm{M})]$;

- temperature-dependent reactivity feedback coefficients as affecting core transient behavior $(\mathrm{H}, \mathrm{M} / \mathrm{L})$;

- temperature-dependent reactivity feedback coefficients (fuel, moderator, reflectors) as affecting passive safety shutdown characteristics $(\mathrm{H}, \mathrm{M} / \mathrm{L})$;

- $\quad$ power and flux profiles as initial conditions for accidents involving potential fuel failures $(\mathrm{H}, \mathrm{M})$;

- positive reactivity insertion and reduction in control worth due to steam-water ingress accidents (H, M);

- control and shutdown rod worth and reserve shutdown worth as required for hot and cold shutdown $(\mathrm{H}, \mathrm{M})$;

- sudden positive reactivity insertion due to pebble core compaction (packing fraction) due to earthquake (M, M);

- xenon-135 buildup and oscillation as affecting core transient behavior (M, M); and 
- xenon-135 and samarium-149 buildup and worth as affecting time to recriticality during loss of cooling with ATWS (M, M).

\subsubsection{Design Aspects That Impact Neutronic Phenomena}

Both the PMR and the PBR are characterized by similar reactor physics design aspects that impact or define the phenomena associated with power level, power distribution, and reactivity control, including shutdown margins. These key design aspects include the following.

\section{Tall, Thin, Annular Cores with Inner and Outer Radial Reflectors}

This type of core tends to be neutronically de-coupled axially and azimuthally in spite of the long neutron diffusion length. The impact of control rod misalignments on xenon-135 oscillation and potential stability problems needs to be quantified during start-up testing. The fuel loadings of PMRs and PBRs are accomplished by very different means, but in both cases the measures used attempt to have axial power peaked near the top of the down-flow cores, so the higher power occurs where the coolant temperature is low, limiting the peak operating fuel temperature. The PMR achieves this by varying the axial fuel loading and by using burnable poisons if required. The major issue for the PMR is whether improper axial-loading of fuel blocks during refueling can lead to an undetected power distribution anomaly and result in excessive operating fuel temperatures.

In the PBR, the on-line refueling system loads the fresh pebbles and acceptably lower burned, xenon-decayed recycled pebbles into the top of the core to enhance a top-peaked power distribution. However, in the PBR, the radial and azimuthal power distributions in the mixed-fuel pebble bed are not well known, and there are indications from melt-wire tests conducted in the Atomgemeinschaft Versuchs Reaktor (AVR) in Germany suggesting that pebbles near the walls of the reflector experienced unexpectedly high fuel temperatures.

For tests at both PMRs and PBRs, consideration should be given (at least in the first core) to use of high-temperature "in-core" neutron detectors that can provide maps of axial and azimuthal power distributions and core-inner-to-outer-radius power tilts; these detectors would likely be located only in the inner and outer reflectors rather than in the core due to temperature and connection limitations.

\section{Active Reactivity Control and Shutdown Systems Where Control Poisons are Inserted into Penetrations in The Outer and Inner Reflectors}

In both the PMR and PBR, control rod misalignments in the outer reflector during operation would result in azimuthal power tilting that could cause xenon-135-induced oscillations when the misalignment is corrected; however, this needs to be verified by analysis and confirmed by test. In both the PMR and PBR, hot shutdown is to be achieved by inserting the outer reflector control and shutdown rods. In the PMR, cold shutdown is to be achieved additionally by inserting the inner reflector control and shutdown rods. As a back-up shutdown system, the reserve shutdown systems (RSS), consisting of small boronated $\left(\mathrm{B}_{4} \mathrm{C}\right)$ graphite balls in hoppers located above the core, can be actuated (manually) to drop the balls into reflector penetrations.

In the PBR, shutdown is typically achieved with the outer reflector rods inserted (for hot shutdown) and then with the additional actuation of the RSS into the inner reflector penetrations for cold shutdown. Based on the most recent information provided to NRC [4-8] by PBMR (Pty Ltd.), the South African version of the PBR has no inner reflector control and shutdown rods and relies solely upon actuation of the RSS consisting of small absorber spheres (SAS) inserted into holes in the inner reflector. The current version of the Chinese PBR [high-temperature reactor, power module (HTR-PM)] has a smaller cylindrical core (as opposed to annular) and will use only outer reflector control and shutdown elements. 
The restart recriticality of the core to begin heat-up on nuclear heat differs between the PMR and the currently proposed South African PBR. Cold recriticality in the PMR is achieved by withdrawing the inner reflector rods until criticality is achieved, while the PBMR, as most recently presented to NRC, will partially de-fuel, remove the RSS material, and then achieve recriticality for nuclear heatup by an as-yet undefined combination of on-line refueling and withdrawal of the outer reflector control rods.

\section{A Neutronically Transparent Single-Phase Coolant (Helium)}

The helium coolant contributes virtually nothing directly to core temperature reactivity coefficients, unlike both water-cooled and sodium-cooled reactors wherein changes in coolant density or voiding can directly affect the neutronic balance and cause large reactivity feedbacks. Replacing helium with air during an air-ingress event will introduce nitrogen that should have only a small negative reactivity effect due to the relatively large thermal neutron capture cross section $(\sim 1.84$ barns per atom) of the low-density nitrogen in air.

Replacing helium with a hydrogen-bearing compound such as in a steam/water ingress event may produce a more pronounced positive reactivity effect that varies both with the amount and density of water introduced, and between PMRs and PBRs. Steam/water ingress tends to have a positive reactivity effect due to increased neutron moderation and reduced neutron leakage up until and if the ingress amount is sufficiently large that the macroscopic thermal neutron capture cross section of hydrogen ( 0.33 barns per atom) in water overwhelms the positive effects of increased neutron moderation and reduced neutron leakage with parasitic capture of the thermal neutrons.

As to the neutronic difference between the PMR and PBR, the PBR will have a higher carbon-tofissile-isotope atomic ratio such that the PBR is more highly moderated, so that in general the steam/water ingress reactivity effect is likely to be smaller than in the less-well-moderated PMR. However, the PBR would be more susceptible to the negative effect of hydrogen parasitic capture during a large water-ingress such as flooding of the core. In addition, since water ingress, and to a lesser extent steam ingress, into the core and control element penetrations in the radial reflectors, will tend to de-couple the core from the externally inserted control poison by reducing neutron leakage from the core, this reduces the effectiveness of radially mounted control poisons, hence reducing the hot and cold shutdown reactivity margins.

The positive reactivity effects of water ingress during shutdown would be seen first in an earlierthan-expected criticality during restart, if not detected beforehand by moisture detectors. However, even if detected early, the use of nuclear heat would be the only efficient means to dry the graphite fuel and reflector elements, other than completely unloading the core's fuel and removable reflector blocks, which in this case would not allow affected reflector graphite external to the core to dry.

\section{A Solid, High-Temperature Ceramic Neutron-Moderator and Reflector (Graphite and/or Graphitized Carbon)}

The reactivity effects of air and water ingress into voids in the core and reflector graphite are addressed above. With a solid moderator there are no density-change effects on reactivity as in watermoderated reactors and thus, aside from the effect of Doppler broadening of fission and capture resonances in the fuel, there is no temperature-dependent moderator-density effect on the downscattering of faster (higher-energy) neutrons to the thermal-neutron energy spectrum. Also, with a higher-atomic-mass moderator such as carbon, the mean thermal energy of neutrons will be higher than that for hydrogen bound with oxygen in water; that is, graphite will tend to produce a "harder" thermal-neutron energy spectrum than would water-moderated systems at the same temperature (assuming no voiding in the water-moderated system). 
Thus, the moderator temperature-dependent reactivity coefficient (MTC) in the PMR and PBR depends upon the change of the thermal-neutron energy spectrum with temperature caused by the temperature-dependent change in the thermal-neutron scattering kernel. ${ }^{1}$ The sign and magnitude of the MTC will depend upon how the change in the thermal-neutron energy spectrum impacts the relative thermal-neutron reaction-rates with

1. parasitic neutron poisons due to low-level impurities in the moderator and fuel pellet binder, where capture cross sections tend to vary inversely with the velocity of the thermal neutron $(1 / \mathrm{v})$;

2. FPs in the fuel with large thermal-neutron capture resonances such as xenon-135 and samarium-149, or with thermal-neutron capture resonance tails such as with rhodium-103;

3. fuel isotopes with thermal fission-capture resonances such as plutonium-239 and to a much lesser extent uranium-235;

4. fuel isotopes with thermal-capture resonance tails such as with plutonium-240;

5. lumped burnable poisons such as boron pins that tend to have black flat neutron-capture cross sections varying little over the thermal-neutron energy spectrum; and

6. possibly distributed burnable poisons (such as gadolinium or erbium) that would have thermal-resonance capture structures.

The MTC is slightly delayed (compared to the fuel Doppler coefficient) but near-prompt during normal powered operation, but it can have large positive and negative contributions from the competing effects discussed above. It is not possible to simulate all of these effects separately in a critical experiment, and difficult to do separate effects measurements in a reactor operating at power. The reflector contribution to the MTC during shutdown without active cooling can be a positive reactivity effect.

Finally, another minor aspect of the use of graphite, instead of metallic in-core structures as in water-moderated reactors, is the fact that, during shutdown, the decay heat power distribution, which is dominated by the capture of decay gamma rays principally in the higher mass atoms with more electrons, is flatter than in LWRs since the decay gammas will tend to transport through the lowdensity, low-atomic-mass carbon and deposit energy in the more widely dispersed, higher-density heavy-metal atoms in the fuel.

\section{Fuel Consisting of Carbon-Coated Fuel Particles Dispersed in a Graphitized Carbon Matrix}

The coated fuel particles are either relatively closely packed in pellet form, as proposed for the PMR, or more widely dispersed in the graphitized matrix material in the pebble, as proposed for the PBR. In addition, the PMR fuel pellets may use mixtures of fissile (enriched uranium) and fertile (natural or depleted uranium, or thorium) coated particles and varying fissile particle enrichments. The diameters of fuel kernels usually vary inversely with enrichment, where the diameter decreases with enrichment based principally on optimizing the strength of the silicon carbide pressure vessel against fission gas pressure with burnup. More highly enriched fuel kernels have a higher number of fissions, and the smaller particle has a stronger pressure vessel provided by the shell of the tighter silicon carbide coating. Larger lower-enriched particles have fewer fissions, a lower fission rate, and

\footnotetext{
${ }^{1}$ As used here the word "kernel" refers to the common usage by physicists in referring to the thermal neutron scattering probability distribution for a neutron to be scattered to another thermal energy where that probability distribution depends on the energy of the incident neutron, the mass and temperature (vibration frequency) of the atom off which the neutron scatters, and the physical restraint of the scattering atom based on its compounding or crystalline lattice bonds to other adjacent atoms. Elsewhere, the term "kernel" usually refers to the inner fuel portion of a coated fuel particle. Therefore, the use and meaning of the word "kernel" in a reactor physics discussion of PMRs or PBRs depends on context.
} 
more volume in the fuel kernels to accommodate FP swelling, so the larger-diameter but weaker silicon carbide shell is adequate for the lower fission gas pressure.

These double or, in the case of the fissile/fertile two-particle system, triple heterogeneities must be accounted for in calculating the neutron reaction self-shielding effects in both the resonance or epithermal region and the thermal region of the neutron energy spectrum to properly calculate the Doppler fuel temperature coefficient of reactivity and the MTC. The mix of fertile and fissile particles in a two-particle system is selected to optimize the reactivity lifetime of the PMR fuel cycle and the utilization of the plutonium produced during the life of the fuel elements over several cycles.

\section{Alternative Means to Accommodate ATWS}

Typical of gas-cooled reactors throughout the world, the control rod drives for the PMR and PBR usually consist of control rods suspended on cables that are wound and unwound from an electricmotor-driven winch, where a scram consists of releasing the motor to let the winch unwind the cable as gravity pulls the rods into the reflector penetrations.

The RSS is, as described above, a set of hoppers with remotely actuated drop doors located above the core and with each hopper filled with small boronated balls.

Water-ingress events experienced at Fort St. Vrain led to two partial-ATWS events caused by moisture and particulate matter being carried through the helium purification purge system, deposited both on the wound cabling on the winches and on the winch motor bearings, thus preventing the free motion of the rods to drop into the core under gravity. Similarly at Fort St. Vrain, water-ingress through the helium purification system led to the leaching of the $\mathrm{B}_{2} \mathrm{O}_{3}$ contaminant in the $\mathrm{B}_{4} \mathrm{C}$ of the RSS balls, allowing the formation of boric acid crystals on the RSS balls sufficient to prevent some of them from falling out of the hopper during technical specifications surveillance testing.

While preventive measures based on lessons learned can be implemented to minimize the possibility of ATWS by these causes that affected Fort St. Vrain, the passively safe PMR and PBR, as demonstrated in AVR and HTR-10 tests, can rely on the combined reactivity effects of xenon-135 buildup, the Doppler coefficient of reactivity, and the MTC to achieve hot shutdown of the reactor immediately upon a loss of cooling combined with ATWS.

During an extended loss of cooling with ATWS, the core will go critical again after the xenon-135 decays, and the peak fuel temperature in the recritical core will depend upon the recritical core power level that balances the rate of passive heat transfer out of the core to the RCCS and the reactivity feedbacks of the Doppler coefficient and the MTC, where the more negative the feedbacks, the lower the equilibrium temperature of the recritical core.

Since the use of the term "safe shutdown" in the Code of Federal Regulations (CFR) - 10 CFR part 50-imposes the requirement for subcriticality to meet regulatory requirements for hot and cold shutdown (see the evaluations of hot shutdown and General Design Criterion 26 in the Technical Evaluation Report of the Nuclear Design of the MHTGR [4-4]), the plant operator retains the safety function of achieving long-term hot and cold shutdown during an extended ATWS, and the equipment used by the operator to carry out this safety function, whether located in the control room or in a remote location, must be appropriately qualified to execute that safety function.

This was an issue for DOE in the NRC preapplication review of the MHTGR [4-3] where DOE proposed a "walk-away-safe" design that was claimed not to require rulemaking to license but did not automatically scram the metallic-clad inner reflector shutdown rods to ensure long-term hot and cold shutdown during an extended loss-of-cooling event. Thus, contrary to the DOE position that the operator had no safety function and that the RSS actuation system did not have to be qualified as safety related, the operator would have to actuate the RSS on a delayed basis to ensure cold shutdown without an uncontrolled recriticality. If the outer reflector control and shutdown rods had failed to 
completely insert at the beginning of the accident in the MHTGR, the operator would also have to actuate the RSS in the MHTGR to ensure hot shutdown in an extended loss-of-cooling accident with full or partial ATWS. Therefore, if the designers of the PMR or PBR want to eliminate the safety function of the operator for this type of ATWS, then supporting data will likely be required to ensure that an uncontrolled recriticality following an accident would be acceptable.

\subsection{EXPERIMENTAL AND ANALYTICAL MODELING ASPECTS OF PHENOMENA}

Sections 4.3.4 and 4.3.5 of the draft Safety Evaluation Report (SER) of the steam-cycle MHTGR [4-3] provide the goals of the MHTGR reactor physics development and qualification program. Because of the greater likelihood of water ingress during operation of the proposed steam cycle MHTGR, additional information was needed on water ingress effects on reactivity and control rod worth. Other information needs included the planned capability to detect power distribution anomalies, verification of the effects of plutonium buildup on the end-of-cycle temperature coefficients of reactivity for the low-enriched uranium (LEU) fueled core, and the uncertainties in the prediction and measurement of reactor physics parameters. The NRC requested that a reactor physics technology development plan topical report be developed and provided for staff review prior to the start of licensing activities. As discussed in Sect. 4.3.5.B of the SER, the experimental and analytical modeling of the MHTGR as of 1988 required additional work.

The experimental data reviewed for the 1988 MHTGR SER [4-3] consisted of reactor data from Peach Bottom 1 and Fort St. Vrain and critical-experiment data obtained by Gulf General Atomics in the 1960s in connection with its large HTGR development program, as well as by Battelle Northwest Laboratories as part of a USAEC-funded program. In addition, LEU critical-experiment data from France (MARIUS III) and the United Kingdom (HITREX) were reviewed. Three findings were (1) there is a paucity of relevant experimental data; (2) there is a lack of documented analysis of the existing data using the analytical methods employed for the MHTGR nuclear design; and (3) comparisons with the British computer code WIMS-D did not provide a basis for acceptable validation.

NRC staff conclusions in the SER [4-3] were based on results of the findings in the supporting Technical Evaluation Report of the Nuclear Design of the MHTGR [4-4].

To assess the current planning by NGNP design proponents to address the safety-related aspects of reactor physics and nuclear design issues, the following documents were reviewed.

1. DOE-HTGR-90348, Reactor Physics-Development Plan, Revision 0, December 1992 [formerly designated as Applied Technology; this document, while submitted to NRC, has not been placed in the Public Document Room. A hard copy of DOE-HTGR-90348 was briefly reviewed by the authors of this report at the DOE Office of Scientific and Technical Information (OSTI) since the document had not been but now is being reviewed by OSTI for Official Use Only (OUO) content to authorizing public release.]

2. DOE-HTGR-90406, MHTGR Nuclear Physics Benchmarks, Revision 0, February 1994.

3. Section 4.0, "Reactor Physics," FSV Experience in Support of the GT-MHR Reactor Physics, Fuel Performance, and Graphite, GA-A-21925 (CONF-9411153-4), General Atomics, November 1994.

4. IAEA TECDOC 1249, Critical Experiments and Reactor Physics Calculations for LowEnriched High Temperature Gas Cooled Reactors, International Atomic Energy Agency, Vienna, 2001.

5. IAEA-TECDOC-1382, Evaluation of high temperature gas cooled reactor performance: Benchmark analysis related to initial testing of the HTTR and HTR-10, International Atomic Energy Agency, Vienna, November 2003. 
6. ANL-05/05, Preliminary Assessment of Existing Experimental Data for Validation of Reactor Physics Codes and Data for NGNP Design and Analysis, Argonne and Idaho National Laboratories, September 15, 2004.

7. INEEL/EXT-04-02293 Rev. 0, Next Generation Nuclear Plant-Design Methods Development and Validation Research and Development Program Plan, Idaho and Argonne National Laboratories, September 2004.

8. Section 4.0, "NGNP Design Methods Development \& Validation," INEEL/EXT-0502581, Next Generation Nuclear Plant Research and Development Program Plan, Idaho, Oak Ridge, and Argonne National Laboratories, January 2005.

Sections 4.0 and 5.0 of document number 7 above describe the current analytical models available to perform reactor physics analyses of the PMR and PBR from the preparation of nuclear cross sections to reactor kinetics modeling. Section 4.2 of document number 7 and Sects. 4.1 and 4.2 of document number 8 set out a logical set of steps for qualifying the analytical modeling of PMR and PBR reactor physics phenomena, given the limited amount of relevant experimental data available. These steps rely upon intercode comparison of neutronic benchmark calculations and analyses of existing experimental data from both zero-power critical experiments and power reactors.

Unfortunately, the zero-power critical experiments performed for graphite-moderated lattices at both room temperature and heated conditions suffer from problems not seen in LWR experiments wherein, for LWR lattices, the dominant effects of the Doppler coefficient of reactivity and the moderatordensity coefficient of reactivity can be measured by separate effects testing.

Graphite-moderated critical experiments have been harder to perform, because the earlier (1940s and 1950s) testing for fuel-rod lattices was primarily done with exponential, not critical, experiments, and the documentation for these lack detailed information about graphite impurities that contribute to parasitic neutron capture. Unfortunately also, the earlier power reactor data sets from Peach Bottom Unit 1, Fort St. Vrain, AVR, and thorium high-temperature reactor (THTR) are for nonannular cores using highly enriched-uranium/thorium (HEU/Th) fuel systems that are not as relevant as more recent physics test data from the smaller, nonannular HTR-10 and HTTR LEU-fueled prototype experiments. The initial HTTR cold critical tests, performed with a simulated annular core, are well documented in an IAEA TECDOC (document number 5).

The older data sets for the gas-cooled power reactors are typically less well documented and therefore are of uncertain quality. Critical-experiment data for heated testing are also of limited value [the Japanese very high-temperature gas-cooled reactor (VHTGR) only reached $200^{\circ} \mathrm{C}$ ]. The HTLTR tests for $\mathrm{HEU} / \mathrm{Th}$ and $\mathrm{Pu} / \mathrm{Th}$ systems were based on determining central region worth of a central heated test article in a zero-power graphite critical pile, where the spatial uniformity, or flatness, of the flux spectrum at the edges of the test region was based on foil activations with spectrum unfolding based only on two-group diffusion theory.

While it is valuable to use the set of zero-power and power-reactor critical experiment results for LEU fuel to partially qualify codes and methods to be used in NGNP predictions, the uniqueness of the configuration (tall, thin annular core) of the current PMR and PBR designs and the high operating temperatures require detailed reactor physics testing of the first unit as a function of core burnup and of the start-ups of the second and perhaps third cycles. Attention should be paid to the instrumentation needs for these tests since neutron sensors must be both distributed and intercalibrated to infer power distributions in the large cores. Neutron detectors used in test measurements should also be sensitive enough to measure reactivity and changes in flux levels and distributions at neutron levels below the point of adding heat. This avoids control rod worth measurement contamination by thermal feedbacks in period measurements. There have been previous efforts to develop reactivity inference techniques that allow simultaneous evaluation of the 
feedback reactivity coefficient with the rod worth measurement, but these inverse reactivity methods have not yet been developed sufficiently [4-9].

Unfortunately for the graphite-moderated gas-cooled reactors, unlike LWRs that can heat up to isothermal conditions at operating temperatures in the core on the combination of heat generation by the reactor pumps and pressurizer heaters, the PMR and PBR must heat up primarily on nuclear heat. Thus, in general, there will be small reactivity contributions from concentrations of xenon-135 buildup and less than isothermal conditions in the fuel and core.

Truly clean reactor physics testing at near-operating temperatures is likely not possible in these reactors if the reliance is on ex-vessel neutron detectors. Thus in large cores, spatial redistributions of the neutron flux must be integrated out of the ex-core detector response to ensure a reasonably accurate measurement of small reactivity changes effected by the rod motion before the response is contaminated by temperature-dependent reactivity feedbacks.

So for the PMR and PBR, isothermal zero-power tests of critical experiments and start-up cores are likely limited to low temperatures (probably less than $300^{\circ} \mathrm{C}^{2}$ ). All higher temperature and power-range tests on the reactors will have to be performed with well-instrumented systems to get more than just the critical position and inlet and outlet gas temperatures, from which all other reactivity effects will have to be calculated or inferred without direct measurement.

Measuring the effects on power distributions of azimuthal rod misalignment and recovery from the misalignment, and the effect on xenon-135 oscillations or stability, requires intercalibrated neutron detectors to measure the azimuthal power response. Interpreting the results of xenon-135 testing likely requires either a three-dimensional time-dependent neutronics code with coupled thermal-hydraulic feedbacks, or a quasi-static kinetics code with a reactor physics model capable of calculating the first- and second-harmonic flux distributions of the fundamental mode.

In transient analyses, point kinetics approximations are often used for the neutronics model because the three dimensional flux shape transient effects in the core during postulated accidents (other than rod ejections and possibly azimuthal or axial xenon-135 oscillations) are relatively small, especially when compared to those in LWRs. Multidimensional neutronics codes are necessary for providing steady-state power peaking distributions for accident codes that use point-kinetics or onedimensional neutronics models. For ATWS events, the dynamics of xenon-135 and samarium-149 poisoning are considered. These codes are sometimes also used in combination with the overall system $\mathrm{T} / \mathrm{F}$ codes.

\subsection{CLOSING THE GAPS-STAGES OF MODEL DEVELOPMENT}

\subsubsection{Near-Term Approaches}

NRC is encouraged to participate in collaboration with the Japanese HTTR and Chinese HTR-10 test programs to plan and obtain reactor physics test data that are most relevant to the needs of understanding key reactor physics phenomena in the PMR and PBR:

- isothermal MTC at higher temperatures,

- xenon-135 worth and stability testing,

\footnotetext{
${ }^{2}$ As described in Sect. 10.2.6 of the Fort St. Vrain Updated Final Safety Analysis Report, the oil-fired auxiliary boiler and the outside oil-fired backup auxiliary boiler could provide steam to the steam generators to heat up the core to slightly over $300^{\circ} \mathrm{C}$ on nonnuclear heating. The HTTR startup testing reached similar temperatures on heat addition from the circulators. Higher-temperature heating of the first PMR or PBR core could be provided (1) if the plant is located near a high-temperature steam source such as a fossil-fired power plant and (2) if the steam can be routed to the shutdown cooling system heat exchanger so that nonnuclear heating of the core can be provided using the shutdown cooling system circulator.
} 
- control rod worth and the effect of core power tilting on worth,

- neutron detector sensitivity and thermocouple instrumentation testing at operating temperatures,

- in-core power distribution measurements,

- transient testing to benchmark kinetics codes, and

- $\quad$ safety demonstration tests (including ATWS).

\subsubsection{Intermediate- and Long-Term Approaches}

NRC should continue collaborating with HTTR and HTR-10 test programs and plan with DOE the reactor physics startup test needs and instrumentation requirements for the NGNP. NRC should consider collaboration with the U.S.-Russian Fissile Materials Disposition Program on heated critical experiments to be conducted in the ASTRA facility at the Kurchatov Institute in Moscow.

\subsection{SUMMARY}

Past reactor physics testing of HEU/Th HTGRs is typically not relevant to the NGNP. The current test plan proposed by the technology development plan for the NGNP, as documented in report number 8 in list in Sect. 4.2, is an acceptable start, but more planning is required for the reactor physics testing during NGNP start-up and the operation of the first several fuel cycles. This is because zero-power testing in the facilities identified in this report is unlikely to be a satisfactory analog to the reactor configurations and operating temperatures of the NGNP, since these are lowtemperature, and cylindrical not annular. More benefits would likely accrue from collaboration on tests in foreign programs as noted in Sect. 4.3.2.

The listing of neutronics issues in Sect. 4.1.1 notes that in the accident-T/F PIRT exercise, there were no phenomena rated high importance-low knowledge $[\mathrm{H}, \mathrm{L}]$, and the conclusion here is also that there are no very significant gaps that must be considered; however, there are several items that do require careful attention to detail in the design and testing programs.

\section{REFERENCES}

4-1. D. R. Vondy and T. B. Fowler, "Solving the Uncommon Nuclear Reactor Core Neutronics Problems," Nuclear Science and Engineering 83(1), 100-111 (January 1, 1983).

4-2 W. M. Stacey, Nuclear Reactor Physics, Second Edition, Wiley-VCH Verlag Gmbh \& Co. KGaA, 2007.

4-3 P. M. Williams et al., Draft Preapplication Safety Evaluation Report for the Modular HighTemperature Gas-Cooled Reactor, Nuclear Regulatory Commission, Washington, DC, NUREG-1338, March 1989.

4-4 D. L. Moses, ORNL Technical Evaluation Report for the U.S. Nuclear Regulatory Commission: Nuclear Design of the Standard MHTGR as Presented in the Preliminary Safety Information Document (PSID), NRC Project Number 672, NRC Public Document Room Accession Number 8903220327, April 30, 1988.

4-5 D. A. Copinger and D. L. Moses, Fort Saint Vrain Gas Cooled Reactor Operational Experience, NUREG/CR-6839 (ORNL/TM-2003/223), Oak Ridge National Laboratory, January 2004.

4-6 R. N. Morris et al., TRISO-Coated Particle Fuel Phenomenon Identification and Ranking Tables (PIRTs) for Fission Product Transport Due to Manufacturing, Operations, and 
Accidents-Main Report, U.S. Nuclear Regulatory Commission, NUREG/CR-6844, Vol. 1, July 2004.

4-7 S. J. Ball and S. E. Fisher, Next Generation Nuclear Plant Phenomena Identification and Ranking Tables (PIRTs)—Volume 1: Main Report, NUREG/CR-6944, Vol. 1 (ORNL/TM2007/147, Vol. 1), Oak Ridge National Laboratory, March 2008.

4-8 J. Slabber, "PBMR Reactor Unit and Main Support Systems," presentation to NRC public meeting at the PBMR Safety and Design Familiarization, February 28-March 2, 2006, NRC Public Document Room Accession Number ML060680079, March 13, 2006.

4-9 R. Fuge and D. Ziegenbein, "Method for Determining the Differential Reactivities of Power Reactors," Kernenergie 16(8), 245-248 (August 1, 1973). 



\section{FUEL AND FISSION PRODUCT TRANSPORT}

This section provides a background of modular HTGR fuel and FPT modeling and historical information about FPT codes and experimental V\&V efforts for HTGRs. The phenomena are identified, and those that need to be addressed are described for each of the significant areas identified in recent PIRT exercises [1-1]. Where gaps are evident, data needs are compared with the data and tools currently available, and potential solutions for addressing the perceived gaps are noted.

At present, there are three required FPT model applications:

1. Prediction of Releases from Anticipated Operational Occurrences (AOOs): 10 CFR Part 50, Appendix I, limits (for LWRs) the annual atmospheric releases to 10 milli-Rem (mR) gamma-ray or $20 \mathrm{mR}$ beta whole-body dose and to $15 \mathrm{mR}$ to any organ from all pathways. This may be interpreted for the NGNP as a limitation on releases from operational events that on the average would occur more than once in the life of the reactor $\left(>2.5 \times 10^{-2}\right.$ per reactor-year, assuming 40-year licensed lifetime, or $>1.7 \times 10^{-2}$ per reactor-year, assuming 60-year licensed lifetime). Whatever these events may be, models or hard data for estimating these releases must be available to demonstrate compliance with the limits of 10 CFR Part 50, Appendix I .

2. Prediction of Accident-Caused Releases: Estimates of public exposure resulting from accidents will be based on models from at least three other disciplines.

i. Reactor thermal-physical models, describing the temperatures, flows, and structural conditions following the event driver. In the case of Generation IV reactors such as NGNP, a major design objective is to employ passive safety features that limit reactor responses in all plausible accident scenarios such that no significant fuel failure and FP releases occur. This needs to be considered as a mitigating factor in assessing potential FPT and releases outside the confinement area.

ii. Fuel behavior models, describing the effect of the accident conditions on the physical and chemical integrity of the fuel.

iii. FPT models, describing the rate of movement of FPs from their normal location in the fuel particle and from their distributed normal operation locations in the reactor circuit to and through the reactor pressure boundary breech and confinement. This topic includes both design-basis and beyond-design-basis accidents. Past HTGR practice has been to apply the lower Protective Action Guidelines (PAG) limits to the Exclusion Area Boundary (EAB) rather than the 10 CFR Part 100 specifications to eliminate the need for emergency planning beyond the EAB. This approach limits the whole body dose to 1 Rem $(\mathrm{R})$ and the thyroid dose to $5 \mathrm{R}$ for accident frequencies as low as $10^{-6}$ per reactor-year. However, this logic should be reviewed in light of the regulatory document revisions that have taken place since the early 1990s.

3. Prediction of Maintenance Doses: To plan for worker safety and plant maintenance operations, a reasonable estimate of the reactor system contamination levels is required. This estimate will also affect the waste disposal and reactor maintenance strategies.

All three applications require a prediction of the FP distribution within the reactor system as a starting point as well as the stability and mobility of this initial distribution. They also require a prediction of the fuel failure rate. 


\subsection{FISSION PRODUCT AND RADIOACTIVE MATERIALS TRANSPORT PHENOMENA}

The principal phenomena of HTGR FPT and radioactive materials transport include

- FP retention in or transport out of the fuel kernel of a coated particle,

- FPT through particle coatings,

- FPT through or sorption in fuel matrix and fuel block graphite,

- FPT around primary circuit and sorption of reactor system alloys,

- removal of deposited FPs from primary circuit alloys,

- transport mechanisms of tritium,

- transport mechanisms of heavy metals, and

- FP and radioactive materials transport into and through the reactor cavity.

\subsection{EXPERIMENTAL AND ANALYTICAL MODELING ASPECTS OF PHENOMENA}

\subsubsection{Background and History of FPT Model Development}

A wealth of data and transport models for the gas-cooled reactor community has been collected and developed over the decades, and this information inspires confidence that the NGNP concept will have benign accident behavior and very low if any releases from AOOs or design basis accidents (DBA). However, while the data show that it is possible to design a reactor with excellent accident mitigation features, barring unlimited air ingress, it has not been adequately shown that the planned designs will always have releases small enough to preclude significant effluent filtering or containment during a rapid depressurization event. To avoid constructing a high-pressure reactor building, the designers have proposed venting the initial pressure release without filtering on the grounds that the FP content of the gases exiting the reactor building is very low. Once the depressurization has been completed, FP containment becomes less difficult because both the reactor and reactor building are at approximately atmospheric pressure and the main driving force, the highpressure helium, is now absent. A bank of low-flow filters can then handle the modest flow demands and the expected small FP releases from the reactor system. Water or air ingress into the reactor can also occur and complicate the situation, but the lack of a strong pressure difference to drive FPs out of the reactor or reactor building is advantageous.

For this design approach to proceed, one must show that the FPs available for release and their transport path result in only very small releases under the possible accident conditions. Thus, the designers must show that the source term, the transport mechanisms, and the accident configurations are well understood, are supported by data and testing, and that sufficient margin is available to encompass uncertainties.

Currently, designers and regulators are assessing the gaps in the knowledge base along with the proposed designs so that resources can be assigned appropriately. At present, the major areas of concern for a fuel and FP transport gap analysis are

- physical models and the supporting mathematical methods,

- relevant data on materials or components over the range of interest and data uncertainties (single effects testing),

- reactor component and confinement/containment configuration and their relative roles in the safety case,

- computational software or other methods for determining the quantitative results, and 
- integral testing over a wide range of conditions to support the development of computational methods and the quantification of the data and associated uncertainties.

In the past, the DOE-funded HTGR program has approached this need by producing a set of design data needs (DDNs) and a V\&V plan that identified data issues that needed to be resolved to minimize design risk (Appendix A in Ref. 5-1 is an example of the DDN approach; Ref. 5-2 is a somewhat dated but informative V\&V plan; Ref. 5-3 is brief, generic, and not very specific). For the most part, little progress was made on these DDNs and V\&V plans over the years; they do, however, serve as starting point for a gap analysis since they reflect past thinking on the needs and problems. A more recent approach is outlined in Ref. 5-4, and Ref. 5-5 outlines the top-down approach toward safety.

The major emphasis has been on data; there was less emphasis on the development of physical models probably because the lack of data for even the simple models made work on the more sophisticated models of limited benefit, and the simpler models appeared to be adequate for the needs of the time. In principle, a large and robust database would encourage the comparison of different models, but the lack of data and the difficulty of collecting the relevant data forces one toward models with sparse data needs. This often pushes designers toward models that produce conservative results rather than models that accurately follow the physics [5-6]. Such models become less useful outside of their narrow empirical boundaries.

A past approach has been to specify that a representation (fission metal transport in graphite, for example) should model the data from an experiment (and a series of experiments) within a factor of 10 to a certainty of $95 \%$. It is not clear whether this approach will be acceptable in the current environment because the demand for model fidelity may be higher and it may not fit within the accuracy needs of the NGNP; again, a design and safety philosophy is required. For example, to inspire confidence in the basic physical understanding, the regulators may require that the model follow the data trends (increases and decreases as a function of key parameters) rather than just be within a factor of 10 of the actual answer [e.g., over the range of $x$, the constant value 4.0 is easily within a factor of 10 of the function $2.0+\cos (x)$, and is a conservative limit, but completely lacks the oscillatory information, leaving one to wonder if the underlying physics is correct].

Another issue is the compounding of uncertainties. If a six-step transport process has a factor of 10 in uncertainty at each step, the final answer could have a final uncertainty of six orders of magnitude. While such a large uncertainty may not be likely, it serves to show that a multistep transport process can compound uncertainties, and the overall path must be considered when evaluating individual models. Because of the desire to use some variation of a vented confinement, a designer cannot simply use bounding assumptions; significant credit must be taken for FP holdup in the reactor system. This drives the need for a creditable transport model.

Transport equations tend to encompass some difficult physics and mathematics, and these equations must be coupled with the varying thermo hydraulics of the core to allow a realistic solution [5-7]. Between data needs, mathematics, and software development, a rather difficult and expensive program is generated. Final verification of the software and models may require an in-pile loop, a difficult and expensive test with many practical limitations. Thus, there are obstacles to developing an accurate, practical transport computational package that allows one to take full advantage of the FP retaining capability of the reactor system components. In addition, an accident can introduce mechanical effects such as shocks, vibrations, acoustical noise, and component fragmentation, which may add uncertainties to the prediction of dispersion forces. The shocks and vibrations are of particular relevance in the NGNP case because a significant portion of the FPs available for release may be either contained in a thin dust layer on the component surfaces or in a friable surface film. Re-entrainment of this dust in the existing gas stream and fracturings of the surface film are transport mechanisms that need to be considered if the coolant is to be released directly to the environment. 
In the case of the NGNP, economics limits the FPT attenuation strategy [5-8, 5-9]. A confinement capable of extensive filtering of a depressurization accident would require a robust building and very large efficient filters which would probably have severe cost consequences. Likewise, containment with an accompanying vent-filter would be a large expensive structure [5-10 through 5-12]. Thus, there is a need to first reduce the core-material FP load by pushing fuel performance to its limit and, second, a need to show that the FPs largely remain in the reactor system. If possible, these two factors will greatly limit the FPs entering the reactor building and, if favorable enough, will require the building to play only a minor role in the attenuation of FPs prior to a (direct) release of the core helium to the environment. In the past, DOE had developed a strategy for FP release and the role played by each component; the high cost of an efficient confinement or containment and the upper limit on fuel quality pushes one toward taking substantial credit for FP holdup in the core and reactor internals. This is the genesis of the FPT program - a replacement of expensive physical structures with extensive (favorable) knowledge about the FP behavior and transport under all conditions of interest.

Complex models with great needs for data and testing are not required if the designer can show that the simpler models bound the physical value for all conditions of interest, but this requires more than just inserting an arbitrary factor to cover the gap. It requires some analysis showing that the physical solution is bounded by the approximation methods chosen; this is difficult in the case of the NGNP because one does not want to resort to a bounding-type analysis, and transport mechanisms cannot be easily compressed into a simple form from which a release envelope can be obtained. One approach may be to frame the problem in a finite time interval. Since the transport processes (diffusion, sorption) are generally slow functions of time and the accident has a finite duration, it may be possible to bound the releases by time intervals. This approach may be more useable than an inventory bounding approach. However, it would not help in the case of dust release or component fragmentation, both of which occur on a rapid timescale.

Maintenance issues also drive the need to minimize and understand FPT in the primary circuit. A low contamination circuit will greatly aid in the maintenance of the reactor circuit and any power conversion components. The ability to predict and control the contamination of the reactor circuit can result in significant cost savings, due to reduced needs for shielding and the ability to conduct handson work.

\subsubsection{Physical Models of Phenomena and Supporting Analytical Methods}

The required functions of any suite of transport models would include the following (see Refs. 5-6 and 5-13 and also see the FPT section in the PIRT main report, Ref. 1-1).

- The nuclides of interest: For diffusive, adsorptive, or vaporization movement, the following elements are important: iodine (I), krypton $(\mathrm{Kr})$, xenon $(\mathrm{Xe})$, cesium $(\mathrm{Cs})$, strontium ( $\mathrm{Sr})$, tellurium (Te), and silver (Ag). Also of great importance is the chemical form of these elements in the reactor circuit. In addition, graphite and coolant impurities may be of interest as they may react with the above elements to produce volatile compounds.

- FP release from the fuel: The failed fuel fraction and any diffusion from intact fuel provide a starting point for the transport analysis. This release is very important because it is effectively the starting point for the transport process and forms the bulk of the material available for release, since fuel failure during accidents is expected to be very low.

- Diffusion, adsorption, and desorption in graphite and fuel matrix materials: After release from the fuel, these are the first materials encountered by the FPs. Sensitivities to 
specific graphite type, neutron fluence, and chemical environment as well as temperature are required. Fuel forms, reflectors, and dust are impacted.

- Adsorption, desorption, and in-diffusion in reactor system metals: FPs plate out on metallic components, and the chemical state of the FP, the surface states, and the temperatures of the component all affect the deposition of material. Radionuclides may be trapped by in-diffusion. Power system components such as heat exchangers may be sinks.

- Chemical and physical forms of the FPs in the coolant: FPs may exist in the coolant as elements, compounds, aerosols, or may be attached to dust. The amount removed per pass of the coolant through the reactor system influences the distribution of FPs and the coolant inventory. Mobile components may exist in an equilibrium that depends on the coolant flow rate.

- Tritium transport models: Tritium is one of the expected isotopes to be released on an annual basis since it permeates through metals at high temperatures.

- Aerosols and dusts that plate-out on reactor system components and their mobility: Mobile and unstable deposits can be re-entrained by changes in the coolant flow rate, by vibration, and by mechanical shocks. These deposits are vulnerable to release by a break in the reactor pressure boundary. If they contain significant activity, they will require holdup by the reactor building (or filters).

- FP reactions with the confinement building materials: Chemical reactions can convert elemental forms of FPs to compounds that are more volatile and biologically active. Iodine is important in this regard.

- Reactions of the reactor system components and FPs with air or steam: Oxidizers change the oxygen potential of the reactor system as well as attack the reactor components. Increased releases can come from both FP chemical reactions as well as the release of FPs embedded in eroded components.

- $\quad$ Plume models that transport the released material beyond the reactor building: Once outside the reactor building, the usual plume models are expected to apply with the understanding that the particle sizes, building humidity (dry helium), and compounds may be different from those of the LWR experience.

The simplest models for use in reactor FPT analysis may consist of vapor transport models based on sorptive isotherms, combined with simple Fickian diffusion for the bulk material, and simple aerosol transport models for dust. Depending on the safety role played by the confinement or containment, this could be sufficient. However, since transport theory encompasses a wide variety of mechanisms that are sensitive to surface conditions and variations in bulk material properties, a simple model may not be sufficient, especially since accidents may lead to significant changes in material properties due to chemical reactions (coolant impurities, air, and steam).

A direct approach using the Maxwell-Stefan equations and chemical reactions may not be practical due to both the complexity of the mathematics and the large amount of data required. In addition, a large pipe break will result in major mechanical forces that can spall off material or damage and fragment brittle components, adding a mechanical component to the analysis.

The mechanical, vibrational, and acoustical forces associated with a large pipe break do not seem to be included in the forces that may generate or re-entrain particles in the exiting gas stream in many of the current literature models, even though the NUREG-0800 guidance specifically lists pipe whip issues as important. Past testing of gas-cooled reactor components has shown that flow vibrations due to normal operation can be important and can even damage components [Ref. 5-14 details some flow induced problems in the British Advanced Gas Reactor (AGR)]. A sudden pipe break can 
change the flow path and flow rates as well as introduce a shock to the reactor system. This offnormal flow situation should be analyzed to determine if the shocks and new flow-induced forces are strong enough to damage or fragment components, and if any vibrations will contribute to the generation and re-entrainment of particles or aerosols into the reactor coolant.

At this early stage in the NGNP development, it is unclear what level of modeling fidelity is required for the reactor circuit, and the regulators cannot evaluate the situation until a conceptual design is available and the safety functions of the reactor system, confinement or containment building, and any filters, are outlined. Also of interest is any mitigation feature that would allow a meaningful response to an unexpected situation either due to an accident or due to problems that turn up during or after construction. One concern with intrinsic safety is that it may not be possible to retrofit or reconfigure the reactor or reactor building should an unexpected problem arise after construction. Long-term experience with LWRs revealed numerous problems that required modifications after construction had begun or was complete.

The following steps would be required to determine the level of physical modeling needed from the NGNP.

- Determine the safety function of each subsystem and the level of FPT attenuation required. Very high levels of FP retention and attenuation under extreme conditions will require a high level of modeling, data collection, and testing; low-to-modest levels of FPT attenuation will allow the use of a coarser approximation and simpler bounding-type testing rather than highly quantitative analysis. The relative costs of safety design options may push the major responsibility for FPT attenuation onto well-understood components, thus avoiding an expensive development effort for the less-well-understood, difficult-to-test components.

- Determine the level of sensitivity to the component uncertainties and how this reflects on the physical models. Highly sensitive models coupled with relatively high data uncertainty may not be very useful.

- Estimate the difficulty in obtaining the data and conducting the testing to support the safety case.

- Scope out how V\&V can be performed.

The conceptual reactor designs presented in the recent PIRT exercises [1-1] allowed direct venting to the environment for a rapid coolant release due to a major pipe break. After a few minutes the vents would close, and some sort of filters (building filters and/or the normal HVAC filters) would be in the circuit to handle the later releases. The building may or may not be of low-leakage design. For analysis and component design purposes, the most challenging design is a higherleakage, vented building design that relies heavily on the fuel and reactor internal components for FP retention, and only a modest amount of building FPT attenuation. This option could require a rather large effort to collect the data and develop the models for analysis.

\subsubsection{Brief Summary of Phenomena Models}

Examples of physical models and brief descriptions of codes are available in Refs. 5-2, 5-6, 5-13, 5-15, and 5-16; also, see the FPT Section of the summary PIRT report [1-1] and the TRISO fuel PIRT report [5-17]. Note that much of the literature is dated material, and currently work is under way to cull and update these codes and models. In addition, there is a wealth of up-to-date computational tools from the fluid flow, LWR, chemical, and diffusion physics communities. Thus, the actual modeling case is probably better and more advanced than indicated by the current and past gas-cooled reactor literature, with the exception of specific materials data and models focused on plate-out. 
The first major issue will be to capture all the relevant physics, coupled with the core thermohydraulics, to produce an integrated model. The second issue will be to collect all the data to support the integrated code suite. The final issue will be to determine and conduct the necessary testing to support the particular NGNP design. These last two issues are likely to be the most difficult, and the ability to conduct them within time and cost constraints may force design, modeling, and data collection compromises.

Several attempts to model experiments have been conducted with generally favorable results, but anomalies are apparent and uncertainties are very high (much greater than a factor of 10) [5-6, 5-13, 5-16]. These anomalies may be due to difficulties in modeling the actual configuration, incomplete data, or effects not in the models, such as surface films, aerosols, or dust transport.

The following sections briefly summarize the state of the models as presented in the review literature [5-6, 5-13, 5-16, 5-17]. This should be considered an introduction with the caveat that proprietary models and data may be available to supplement or supersede this material. The reader may notice that the major issue is the lack of a robust database for the materials of interest. Much work has been done in the past, but the variability of the materials, the design changes, and the difficulty of adequately characterizing the materials makes extrapolating past data and results suspect, thus the need for more testing.

A host of models exists, both based on empirical data fits and transport theory; the complex environment and the difficulty of collecting data have forced modelers into using simpler models and expressions than one might expect. Often, the database needed to sort out the physics and critically evaluate models does not appear to exist.

\subsubsection{Coated-particle fuel performance models}

Currently fuel performance models are in a state of development. Past models often relied on particle coating "pressure vessel" failure models, which now appear to be too simplistic, although they may be suitable for some specific fuels under well-characterized conditions [5-6, 5-17]. The German code CONVOL predicts very low failure fractions for the German fuel, and the irradiations have seen these low failure fractions; the code and the irradiation did not disagree, but it is not clear if this corresponds to a validation of the code. Post-irradiation examination of particle fuel is difficult because of the large number of particles and the limited ability to locate a flaw within a particle. Conducting an irradiation program to fully examine the fuel envelope and compare the results to the models would be very expensive and time-consuming.

Past U.S. models [the General Atomics (GA) code PISA, for example] were based on a onedimensional multilayer stress analysis that did not appear to be adequate for past U.S. fuel under its irradiation conditions. Current models are based on a detailed stress analysis of the particle coating layers and the interfaces between them. Failures are now seen to be due as much to local stresses stemming from coating interfaces or cracks due to global over-pressurization. These models are currently under development, as are the irradiations they hope to predict. Two of the recent codes are PARFUME and TIMCOAT [5-18, 5-19]. Both codes have been used to explain the differences between the past German and U.S. fuel performance.

Codes predicting fuel performance at high (accident) temperatures, such as PANAMA (and the later modifications), were successfully used by the German program to model the high-temperature behavior of their fuel. This type of approach may be useful for NGNP if a similar fuel type is used and the normal operating conditions and burnups are not much more aggressive. High temperatures and higher burnups can lead to much greater noble metal corrosion of the SiC layer, which can result in a higher failure rate at accident temperatures. The new U.S. fuel prediction codes are expected to include the ability to model accidents, so this work may already be under way. 
A major factor limiting the predictive ability of the suite of fuel performance models is simply the lack of sufficient materials property data for the particular fuel and a robust database. A host of structural, diffusion, and chemical kinetics data is required to do the calculations. Since the current fuel is specified by process conditions as well as measurable properties, the best way to fill these gaps is not immediately known; one cannot easily appeal to theory. The literature values may or may not apply to a particular fuel. At this point, the data uncertainties are probably large. Extensive testing of the actual fuel to be used, followed by backing out the coefficients after testing, may be the only practical approach.

Exactly what is meant by comparing code predictions to experiment needs to be clarified. If the coatings parameters are found by comparing the code results to irradiations rather than by independent measurement of the properties as a function of neutron fluence and temperature (which could be a nearly impossible task), the problem then arises in deciding whether the values computed are actual physical values or simply a set of parameters that make the code work for available data sets. A likely approach is to combine information from several sources: historical, specific measurements, theory, and experimental back-out and then check for consistency.

Once a failed fuel fraction has been computed, another issue pertinent to transport is the behavior of the $\mathrm{PyC}$ and $\mathrm{SiC}$ layers because they form the barrier to FP release, assuming the rest of the particle is well behaved. Diffusion models are used in these layers, but silver appears to move by another mechanism. Diffusion coefficients for $\mathrm{SiC}$ (and PyC) have been measured for several isotopes at the temperatures of interest, but they depend on the microstructure of the material. So far, this dependence on microstructure has not been quantified and is a topic of current interest. Other issues include FP corrosion of the $\mathrm{SiC}$ layer, migration of the kernel, and high-temperature decomposition of the SiC. All these issues have uncertainty associated with them, but they are controllable by regulating the oxygen potential of the kernel, controlling the normal operating temperature, and limiting the accident temperature. Chemical attack of the fuel under oxidizing conditions is also likely. Again, we are back to a database.

The importance of these models lies in the quantification of the source term to be used for both normal operation and accident conditions. A large uncertainty in the source term will propagate through the entire transport chain, so a credible estimate will be necessary to justify the selection of the containment/confinement option.

\subsubsection{Fuel-form mechanical and thermal models}

The mechanical, thermal, and chemical properties of the fuel form (sphere or compact) influence its behavior during normal behavior and under accident conditions. The dimensional change of a fuel compact vs the dimensional change of its hole in the graphite block influences the mechanical stresses on the compact (crushing) and heat transfer (across a gap). Thermal behavior determines temperature drops, and chemical properties determine the behavior under chemical attack conditions (even at normal operating conditions, because of impurity attack).

Models have been developed for past fuel forms with their particular matrix mixes, heat treatment, and final density. For the most part, the dimensional and thermal models are polynomial fits to the data rather than theoretically based. It is not clear that this information will apply to fuel forms with different matrix formulations and densities and exposed to conditions beyond tested boundaries. Chemical activity will probably also be a function of the particular matrix and heat treatment. These properties are likely to be a function of neutron fluence as well.

Unless the current fuel will duplicate past, well-characterized fuel, a data gap is likely, and testing will be necessary to obtain coefficients necessary for the models and to determine the particle damage fraction during fabrication. Chemical activity may be a function of impurities and specific processing. The models will then have to be updated or modified. 
A longer term issue is the availability of the specific materials used to make the matrix. The matrix is a combination of synthetic and natural graphites along with a binder. Since the characteristics of the materials depend on the feedstocks, which can be variable, one would like assurances that significant matrix property changes will not occur over the long term. One option is continuous testing of the matrix and fuel forms, but this can cause schedule delays and additional expense. Other solutions to this problem would be desirable.

\subsubsection{FPT within the kernel}

FPT within the kernel is the first step in the release of FPs. It is most important for crushed or failed fuel particles, because these releases go directly into the fuel matrix. These releases, combined with any uranium contamination, dominate the activity in the reactor circuit during normal operation; at higher temperature accident conditions, their release rate may increase.

The Booth model is generally the starting point for FPT from the kernel. Different models include variations due to the birth point of a FP (grain or pore), recoils (athermal diffusion), trapping, and vacancy migration diffusion. Temperature effects are included as well. The models often include several constants that must be determined by experiments, as well as process parameters such as grain size. Coefficients for the various models have been tabulated for a range of past experiments. However, the results may be sensitive to the exact processing used for the particular fuel (density, pores, grains, chemistry), so verification of a particular model may be necessary for the NGNP fuel. This is indicative of the VHTGR dilemma and will be seen throughout this section; a host of models and data exist, but the variability of materials and process methods, along with the sensitivity of the physical process to the actual configuration, are obstacles in settling the issue once and for all.

Since a host of kernel (fuel) FP release models are available, both from the VHTGR and LWR communities, the major issue will be to observe the releases for the specific fuel kernel, see what model best describes them, and compute the required coefficients or determine if the literature coefficients are acceptable. A fallback position would be to make kernels using a well-characterized process or use known highly conservative release values.

During normal operation, the release rate is significantly less than $100 \%$ and an accurate estimation of the released material will probably result in a lower modeled coolant activity and primary circuit contamination than if one uses a bounding estimate (100\% release). This could have a large effect on the modeled release during a depressurization accident, with the resulting regulatory impact and the need for a means of mitigation.

Generally, FP release is broken into two components, inert gases and metallic elements. Iodine and tellurium are assumed to behave like xenon. The uncertainty in the U.S. modeling gas releases is estimated to be roughly a factor of two with the caveat that much of this model was developed in a TRIGA reactor environment, which may or may not be appropriate for the NGNP. The situation for the metallic elements is not as good with the uncertainty simply listed as "exceeding large" [5-6]. Other international models focused on a particular kernel at the conditions of interest and may be better, provided the NGNP uses a kernel with similar properties under similar conditions [5-13]. The kernel design has not yet been selected.

Because much depends on the kernel grain structure and oxygen potential, the releases can change with burnup. Also, air or water can oxidize the kernel and greatly increase releases. Some data are available for kernel oxidation, and it depends on a host of conditions such as kernel composition, temperature, and burnup.

If specific information is available for a known fuel type, and water ingress is not a concern, the current modeling and data situation may be more tractable for estimating the releases during normal operation; otherwise, such data will have to be collected or at least a specific database confirmed. If 
no data are available, $100 \%$ release from the kernel may have to be assumed for regulatory purposes, with possible design penalties.

\subsubsection{FPT in graphite and matrix material}

Once released from the fuel, the fission gases are assumed to very quickly move through the matrix material and graphite to the coolant; thus, the release model is an instantaneous transfer to the coolant. Metallic FPs are assumed to fairly quickly (instantaneously in the case of fuel compacts) distribute themselves throughout the matrix material. Overall, the matrix is assumed to have little impedance to gas transfer and to have a very high diffusion coefficient for metallic elements (compared to graphite). Graphite is also assumed to have no impedance to gas transport.

Note that the matrix material planned for use in the NGNP will be much more like the matrix material used in pebbles rather than that used in historical U.S. compacts, even if the prismatic core option is used. The transport characteristics are more likely to be like those of the German pebbles or the Japanese compacts.

Graphite has significant impedance for the transport of the metallic FPs. Older models have used simple Fickian diffusion, which is believed to be an oversimplification; the actual mechanisms are believed to include both trapping and diffusion, and new models may include these effects. However, the actual model to use is not clear at this time, and simple diffusion with an effective diffusion coefficient may be good enough in some cases.

Transport in graphite is dependent on the type of graphite, the neutron fluence, oxidation state of the graphite, source concentrations, and impurities which may act as trapping sites. Since the NGNP is likely to use a graphite that differs somewhat from the historical materials, it is very likely that the use of past literature data for other graphite types will introduce great uncertainty into these calculations. Testing of the actual graphite will be necessary to get realistic transport coefficients. While much past gas-cooled reactor modeling used Fickian diffusion, it should not be too difficult to explore the newer, more advanced codes for application to this problem. The downside is that the codes will require more data if one is to include effects beyond simple diffusion such as permeation, trapping, fluence effects, and chemical reactions with impurities. Again, the program is data limited and the data are difficult to acquire. It might be interesting to see if a theoretical release envelope could be determined that would be more conservative than detailed (but difficult) calculations and smaller than a bounding (but large) estimate.

As with the matrix, a long-term concern is the stability of the graphite supply. Graphites are sensitive to the coke source and the processing conditions, so if the safety case for the reactor depends strongly on the graphite transport properties, long-term monitoring of graphite transport properties may be a consideration if coke sources and processing conditions are changeable.

\subsubsection{Fission product sorption in matrix and graphite}

Sorption isotherms used in the models and codes are a combination of the Freundlich and Henrian isotherms. Data has been obtained for some elements; others may be assumed to be similar to their chemical cousins. Matrix material does not appear to be affected by neutron fluence, but oxidation effects are not clear. As usual, the model coefficients could be affected by the particular matrix mix used in the fuel form, so uncertainties may be high if the actual matrix to be used has not been explored.

Unlike matrix material, sorptivity in graphite is a function of neutron fluence, generally increasing. The existing database is incomplete, and some of it is for unirradiated graphite. Again, chemical cousins are often assumed to have similar behavior; this may or may not be acceptable for regulatory purposes. Also, the NGNP graphite may be different from historical materials. 
Two other issues may be important for these models. The first is the approach to equilibrium and whether this relaxation time is important for general calculations or accident conditions, and the second is the FP reactions with graphite impurities. If the approach to equilibrium is reasonably rapid, an assumption of equilibrium conditions may be acceptable since the NGNP is expected to have slow accident behavior. The reaction with impurities is more problematic. Iodine may react with metallic graphite impurities to form volatile metallic iodides, decreasing the ability of graphite to hold iodine. Some preliminary data indicate an effect of orders of magnitude [5-6]. If chemical issues are important, the situation becomes quite complex as one then needs the behavior for a wide range of elements and compounds along with their equilibrium.

The sorptive isotherms are key to modeling the transport across the fuel compact graphite gap and the release from either fuel form into the coolant. Partition coefficients between fuel compacts and graphite blocks can be determined and used to relate compact surface concentrations with neighboring graphite surface concentrations, but they too are sensitive to the isotherms.

Modeling the release into the coolant is determined by a mass transport equation that relates the mass flux into the coolant to the concentration gradient across the boundary layer and the FP desorption at the graphite block or pebble surface. Simulations of experiments have often seen an under-prediction of cesium transport, and other effects such as kinetics or boundary layer behavior may come into play. Some of these effects may be in the codes. The modeling is not straightforward because of these multiple effects, each of which carries an uncertainty into the transport path.

The transport of FPs from the surface of the fuel form to the coolant is a particularly important issue as once the FPs are in the coolant they can be spread throughout the reactor system and move back and forth between other transport intermediaries such as dust and mechanically unstable surface films. It is this mobile and friable material that is subject to release during a depressurization. If the sorption transport rate is low, it will be a welcomed bottleneck in the transport path; if high, increased emphasis will need to be placed on determining the releases into the matrix and graphite material from the fuel particles.

\subsubsection{Transport of fission products and sorption on reactor system metals}

A considerable amount of work has been done in the code area, with the FRESCO and SPTRAN codes for the German program, the FORNAX code for the Japanese program, COPAR, PADLOC, TRAMP, and TRAFIC for the U.S. program, and RADAX for the South African program. Others codes are likely to be under development as well. As a rule, these codes include diffusion transport, sorption, and in some cases, dust to compute the FP distribution in the reactor circuit. The reader will have to refer to the latest documentation (from the code writing institution) for a particular code to understand the details, as the open literature may be a decade or more behind.

It is difficult to rate the V\&V status of these codes and how they might be applied to the NGNP because a particular code may not include all the possible transport mechanisms active in the core. In addition, the lack of specific data and realistic integral experiments limits the application to the NGNP. The actual design of the NGNP may or may not impact the dominant transport mechanisms in the codes; any mitigation means could allow more uncertainty in the transport calculations. If great dependence for the safety case is made on the transport calculations, extensive testing and code work may be required.

One of the major issues is dust and aerosol transport under both normal and accident conditions. Recent models and experiments may shed some light on this issue. The importance of this issue for the NGNP is unknown at present but is likely to be high if reactor circuit retention of FPs is very important to the safety case.

Overall, a framework for the computations, a model subset, and a modest database are available for the transport calculations relevant to the NGNP, both from the VHTGR community and the 
general transport community. Exactly how to apply this information to the unspecified NGNP design is unclear at present, but it is likely that some effort will be required to select the most appropriate models and collect the necessary data. Pertinent to this task is to determine the uncertainty of the calculations, as the demands of more than modest uncertainty ( 10-100 times) could be very high.

Sorption is used in the models to predict mass transfer across the gas/solid boundary, and whether or not this model is appropriate to the NGNP situation is unclear. Other forms of transport, such as dust or aerosol transport or increased volatility due to chemical reactions, might also be included. The full spectrum of mechanisms to be considered is not clear at the present time. Deposition on metal surfaces is modeled as sorption behavior either alone or accompanied by dust. Modeling indicates a virtually $100 \%$ deposition of FP metals per circuit pass, but dust or aerosol transport may be important in actual reactors, leading to a small but significant circulation of FPs in the primary loop. The presence of dust has been shown to modify the deposition profile and could result in fewer FPs in the cooler section of the reactor circuit at the expense of a mobile form of contamination.

Some metals may diffuse into the metals, aiding in the immobilization of FPs; however, the lack of relevant sorption and diffusion data leads to great uncertainties in evaluating plate-out behavior and its mobility. This represents another data gap, but a modeling gap may also exist if sorption behavior models are not adequate. There is very little information for turbine-type alloys and the true importance of the dust or aerosol issue. This problem is particularly acute for silver transport and maintenance of the power conversion system [5-16].

\subsubsection{Removal of deposited fission products from primary circuit alloys}

Depressurization accidents can blow contaminated mobile material out of the primary circuit and into the reactor building. The historical models generally use some variation of the coolant shear stress near the wall to remove and entrain material in the existing coolant. As discussed in the FPT Section of the summary PIRT report [1-1], this is unlikely to be an acceptable model by itself because it does not include the mechanical shocks, vibration, or acoustical noise that would be generated by the structural failure of a large high-pressure pipe. Past experiments were condemned as flawed because lift-off tests were conducted by cutting and removing sections of pipe and transferring them to a different apparatus for lift-off evaluation. Such tests showed much larger lift-off fractions than tests that were conducted in situ [5-6]. The fact that mechanical handling would damage friable surface films and dust bonds seems to have been no part of the discussion, but this is exactly what one would expect with a failure of this nature. Other testing included physically wiping the components with a cloth and saw higher removal rates than by shear ratio changes alone [5-13]. The scatter in the test data is large.

Other FP surface removal effects such as steam and air have been examined to some extent, but the data and testing are sparse. Overall, this should be re-examined in light of the specific needs of the NGNP and the neglect of mechanical actions. In particular, the mechanical effects on surface films and component fragmentation should be investigated, as well as the flow induced vibration effects that may cause further particle re-entrainment or reactor system damage. A massive highpressure pipe failure can potentially cause serious secondary damage.

The potential uncertainties in this area could lead to design compromises if the FP contamination of the reactor circuit is significant. The issues of surface film or deposit spalling and collateral damage need to be addressed more directly.

\subsubsection{Dust and aerosol transport}

Past modeling has indicated that circuit contamination in low-temperature regions is significantly reduced through the presence of dust. This is due to a partial decontamination of the surface by dust desorption and by the binding of FPs to the dust in the gas phase. The final FP distribution can then become complex because of the dust transport, which depends on dust particle size and nature as well 
as on the coolant flow rate and flow rate variations. This is a complex problem because of the competition between the dust and the structural metals for the FPs; mass transport and sorption models will have to include this effect. Absorption of FPs on dust will then make them more mobile in the case of a pressure boundary breech.

There has been considerable progress in dust and aerosol models in the past decade, and these models rather than the historical gas-cooled reactor models may be better starting points [5-20, 5-21]. This area should be re-examined in light of the recent work in this field and the experiments done for LWRs. New models and insights have been developed over the past decade, and this material is not considered in the older VHTGR codes. Since FPs entrained in the exiting helium are so important for release calculations, careful analysis is warranted. A way to conduct bounding calculations would be welcome as this modeling tends to be complex.

Dust generation by abrasion, metal oxidation, oil soot, fast neutron damage, and carbon filament growth will need to be addressed as well to determine the quantity of dust in the circuit. Some kind of model will be necessary to estimate the generation rate and the size of particles. Aerosols may be of interest as well. This is also a difficult area, and the available models may not be sufficient if accurate dust and aerosol measures are required. Estimates of $10 \mathrm{mg}$ per pebble per pass through the core for dust generation lead to many hundreds of kilograms of dust in a pebble-fueled power reactor. Where the material ends up and how it is recycled within the flow circuit is important for conducting transport calculations.

This entire area is another important concern. If a substantial portion of the FP inventory ends up on mobile components such as dust and oxide particles, the vented containment option may require considerable analysis to evaluate its suitability. Lack of a good predictive ability may lead to large uncertainties which could drive the designers away from the vented confinement option and to some kind of effluent filtering.

\subsubsection{Tritium transport}

Tritium is usually more mobile and exhibits different behavior from FPs. It can diffuse through metals at high temperatures and thus becomes part of the normal releases of an operating reactor. SiC retains tritium well, so most of the tritium generated in the fuel comes from fuel with absent or damaged $\mathrm{SiC}$. PyC can also retain tritium, although not to the extent of $\mathrm{SiC}$. Graphite impurities, $\mathrm{B}_{4} \mathrm{C}$ control rods, and ${ }^{3} \mathrm{He}$ impurities generate small amounts of tritium as well.

Tritium release from kernels is often assumed to be $100 \%$ and that from control rods is assumed to be due to diffusive release. It is assumed to have rapid transport through the matrix and have no sorption in graphite at temperatures less than $650^{\circ} \mathrm{C}$, temperature-dependent sorption between $650^{\circ} \mathrm{C}$ and $1223^{\circ} \mathrm{C}$, and desorption above $1223^{\circ} \mathrm{C}$. Sorption is modeled as increasing with neutron fluence. The data for this model are weak, and more recent work from the fusion area might be used to fill in data and model gaps.

The transport of tritium though metals depends on the alloy, the surface film, the temperature, and coolant concentration. Historical models were developed for steam generator materials, and these may not be appropriate for current alloys. These models are dated, and the literature should be reviewed for newer models and materials data.

The tritium generation is not expected to be large, so greater uncertainty may be acceptable in the modeling. It is likely that suitable models and data can be found in the recent literature, so tritium is not likely to be a large gap issue.

\subsubsection{Heavy-metal transport}

The models used for heavy-metal transport are similar to those used for general FPT. The heavymetal transport issue does not appear to be great, as the amount of plutonium or americium transport 
at NGNP temperatures is expected to be very small. Data for transport through the coating layers are very limited, but the diffusion coefficients appear to be very small. Sorption and diffusional transport in graphite at the temperatures of interest are expected to be small. Americium is more volatile, but unless the NGNP is fueled with transuranic elements, heavy-metal transport is unlikely to be a major issue. A review of the recent literature may be appropriate as recent work in fuel recycling may shed some new light on the issue and bring the HTGR community knowledge up to date.

\subsubsection{Reactor building transport}

Once the FPs have escaped the primary circuit and entered into the reactor building, several processes exist for their removal from the building atmosphere. These include condensation, deposition, and settling. These processes have been studied for some time for LWR applications; it is assumed that recent work in this area will be available to the NGNP designers. Potential differences include the dry environment, particle size and shape, and the possible greater importance of electrical charging mechanisms. In the past, codes such as PARDISEKO, CARCAS, CONTAIN, and GOTHIC have been used. It is believed that more modern codes or updated versions of these codes will be used to analyze the NGNP situation, and that a large knowledge gap is probably unlikely.

The transport in the confinement will be sensitive to the actual design of the building. Obviously, a vented building will allow a rapid flow path out of the building and less opportunity for settling, while enhanced containment offers more opportunity for FP removal prior to any gas venting.

As with the LWR case, mechanisms that allow the conversion of inorganic iodine to its organic forms are important and may form a part of the safety analysis case, depending on the building design.

Note that there have been updates to the licensing support documents since many of the past calculations for accident doses have been computed [5-22]. The computational methods and their application to the current regulations should be reviewed as past methods may now be obsolete.

\subsubsection{Relevant Material or Component Data Over the Range of Interest and the Data Uncertainty}

While a considerable amount of data has been collected over the years, much of these data are not directly useful for the NGNP because of NGNP's higher temperatures of operation, the super alloys needed for the power system components, and changes in graphite type [5-5, 5-13,5-16]. The higher temperatures and different materials will likely require a new set of experiments to collect diffusion, sorption, chemical attack, and permeation data. Graphites can have different transport properties due to differences in their coke source and manufacturing, so graphite transport property and air/steam erosion data specific to the design material will have to be collected. Also, the metal alloys of interest are different from those of the past. The previous section detailed some of the data impacts.

One complicating issue is that the transport properties are sensitive to the material surface condition and the chemical form of the FP; helium impurities will likely set the oxygen potential of the system and the species to be included in an analysis. Another difficulty is that associated with aging. One will have to estimate the surface qualities of the reactor system components after many years of operation. Some means of determining the effects of operational upsets will have to be incorporated in the plan to determine if any unusual behavior will occur if water, oil, or some other (decontamination?) fluid is introduced into the reactor circuit. The Peach Bottom HTGR had a carbonaceous film on the reactor circuit components from an oil leak from a helium compressor. This film was believed to modify the cesium sorptivity of the circuit [5-16].

Surface films may have to be examined for long-term growth and friability since they are dependent on FP holdup during accident conditions. The diffusion of FPs into the base metal may be 
advantageous in this respect, although decontamination would be more difficult. The decommissioning of past gas-cooled reactors may supply useful data for this determination.

One item of concern for aging is component replacement. New materials may be used to overcome operational problems, and if FP retention is part of the function of the component, these new materials may have to undergo testing for transport properties as well. This is a potentially timeconsuming and expensive process, so a testing plan that could generate material type data that could be applied over a class of materials would be beneficial to the program.

Of particular interest are any turbine or power conversion components that may have to be decontaminated prior to maintenance. Two situations are of interest. The first is the initial collection of FPs while in the reactor circuit. The second is the decontamination of the component and the new surface state of the component after decontamination, and whether the new surface of the component is more or less transport active.

If a component is called upon to retain FPs during an accident, it effectively becomes part of the reactor safety system, and its long-term ability to retain FPs becomes a matter of concern. Since FP retention is sensitive to surface state and the chemical form of the FP, some means of predicting the long-term stability of this retention behavior becomes desirable. This problem is related to general component aging, but in an unusual way as gas impurities or the mechanical stability of a thin film rather than actual component failure could defeat the component's FP retention ability. Unless the reactor circuit parts are periodically cleaned, the long-term stability of the plate-out, films, and deposits could be a knowledge gap issue if their retention is required for the safety case.

\subsubsection{The Reactor Component and Confinement/Containment Configuration and Their Relative Roles in the Safety Case}

This is probably the major issue in any gap assessment; the respective roles of the reactor circuit and containment or confinement system must be known before their modeling adequacy can be determined. As mentioned earlier, the reactor designers are faced with a knowledge/capital trade-off. It may be advantageous to focus design effort on relatively well-understood components such as filters and structures rather than to enter into the complex world of nuclear component testing under extreme conditions. On the other hand, great costs saving may be possible if credit can be taken for FP retention within the reactor circuit.

In any event, the adequacy of the modeling will depend on the safety functional analysis. Detailed transport calculations may be required for one approach while bounding estimates may be suitable for another. Past trade studies have been conducted for variations on the vented confinement approach, and they tend to point to the great advantage of taking credit for reactor system FP retention; however, the NGNP is not bound to the past, and new approaches may be forthcoming.

Once the source term has been estimated, one needs to budget the FP holdup among the fuel form, reactor circuit components, mobile elements such as dust, and the reactor building. Depending on the particular design, one may be able to use bounding assumptions about transport rather than mechanistic calculations for some part of the path between the fuel form and the environment, although the current approach may not be able to employ this simplification. In principle, this allocation of attenuation and holdup will allow the designer and regulator to concentrate resources on the most important part of the transport path or to shift away from the components that are the most difficult to analyze or test by adding additional attenuation in other components that are easier to analyze and test.

Thus, to begin closing the gap and to proceed,

1. the transport pathway for the accidents of interest should be outlined; 
2. the FPs to be retained and the goal attenuation factor for each step (serial or parallel) along the way should be detailed; and

3. the local (accident) operating environment for each step or component in the path should be determined.

This is perhaps the first gap to be closed - the first details of the actual safety approach. Without this information, the regulators would have to guess as to the safety path to be taken. While the literature and presentations have sketched out a design approach, this may not be the only approach, and this approach requires the collection of a lot of data and extensive testing - at great expense.

\subsubsection{Computational Software or Other Methods for Determining the Quantitative Results}

Over the decades, a host of software has been generated for FPT analysis in gas-cooled reactors [5-2, 5-4, 5-6, 5-13, and 5-15]. Two long-term difficulties have been encountered. The first is simply data collection for the models. Even for the simple models, a large amount of data is required because of the wide range of temperatures, materials, and chemical conditions that may be encountered under both normal and accident conditions. Only a part of this information can be collected out-of-pile, since radiation-induced changes in the materials can affect the transport properties, especially in materials like graphite. In addition, these properties are sensitive to the actual microstructure of the materials, so the use of generic rather than specific material properties is seldom possible. Therefore there is a shortage of data for the particular materials of interest at the conditions of interest.

The second issue is integral testing. The most realistic way to conduct integral testing is in an inpile loop. This type of testing is expensive and has many inherent limitations due to reactor safety and operational issues. Even with these limitations, a fair amount of in-pile testing has been done with mostly reasonable agreement with the models, although there have been some anomalies, and the uncertainties are large [5-6, 5-13,5-16]. This is of particular interest for the V\&V of the computer codes, as it provides an overall test of the models and software.

Over the years, a suite of codes has been developed for FPT analysis by both the gas-cooled reactor and LWR (containment transport) communities. It is difficult to evaluate the suitability of the software, but it is probably safe to say that initially, data for the materials of interest and a method to conduct integral tests under the desired accident conditions are the main problems.

The existing models and codes are likely to be good starting points in most cases unless detailed micro-structural elements are required for the calculations, or accuracy levels better than roughly an order of magnitude are required for the design. To proceed with this topic, the following two requirements must be met:

1. A description of the physical models and the reactor configuration. Much of this is available in one form or another, and the reactor design is forthcoming. A more difficult problem is showing that the models are appropriate for the conditions of interest.

2. The data required for the models. These data are composed of two types: single-effects data for each material and component acquired under individual testing and integral data designed to show that the codes get the correct answer for a complete system under the conditions of interest.

These needs are related to the safety pathway needs because the release and release uncertainty demands will influence the degree of modeling and data collection necessary. In fact, the two interact because one would like to credit the best-understood and least-expensive pathway or component with the most FP attenuation or retention to minimize development time and capital cost.

The major gaps are likely to be in the areas of data collection and proof that the selected model is adequate under all the normal and accident conditions of interest. In the end, even if the transport 
cannot be calculated exactly, one would like a model that envelops the releases, and some reasonable proof that the model predicts, in fact, an upper limit.

\subsubsection{Integral Testing Over a Wide Range of Conditions to Support the Computational Methods and Their Uncertainty}

This task is perhaps the most difficult issue the NGNP designers face-proving that the codes and models reproduce reality. As was mentioned previously, an in-pile loop that can be subjected to operational upsets is likely to be the best means available for integral accident testing. It may be possible to do a very limited amount of accident testing on current test reactors, but safety limitations will probably place great restraints on this activity.

A fair number of in-pile loop tests have been conducted over the years, but a loop suitable for the NGNP program may not be available as most of the past loops are not available. Past testing has revealed reasonable agreement with the codes in many areas, but several tests have had anomalies that could not be explained by the simpler transport codes [5-6, 5-13, 5-16]. Thus, a first area of inquiry would be to use past data to re-examine the current transport models and codes. This may or may not be possible because the relevant data needed by the models may not have been collected.

The planning of any in-pile loop program would require a fairly complete description of the normal operating environment and of the accidents, along with any scaling factors. Rather extensive modeling will be necessary both to design the loop as well as determine the off-normal conditions that the loop can be expected to simulate. Finally, the model predictions (with the previously collected single-effects data) will need to be made.

\subsection{CLOSING THE GAPS-STAGES OF MODEL DEVELOPMENT}

\subsubsection{Near-Term Approaches}

At this early stage in the development of the NGNP, the actual path for the safety case is unclear or how the designers plan to meet the regulations; however, based on a historical approach using the filtered/vented reactor building design, several general gaps are apparent.

1. Regulators need a comprehensive description of the NGNP safety philosophy, a listing of the components involved, and the conditions under which these components are expected to perform their safety functions. The regulators also need an explanation of how this philosophy meets the defense-in-depth approach and, in particular, answers to the following:

i. Will the components that perform a safety function (retain FPs) be classified as safety-related components with the imposition of equipment qualification, in-service inspections, and/or technical specifications limiting conditions for operation and surveillance requirements?

ii. How will aging issues be addressed? If the safety function of a component is to retain FPs on its surface during adverse conditions, how can it be ensured that this function can be retained for long periods (decades) despite the possible presence of other long-term surface degradation mechanisms?

iii. Will the surface state of a nonreplaceable or difficult-to-replace component be reactivated by chemical action or cleaning during its service life?

2. A sound basis for the selection of the physical models and the data for these models must be justified. A host of transport mechanisms has been identified for the NGNP, and unlike other reactor concepts, the safety case greatly depends on the knowledge of these mechanisms under extreme conditions (high temperatures) as a high-pressure 
containment (traditionally) has not been part of the design to contain releases; the internal reactor components themselves perform a major FP retention role during at least part of the accident. While some comparison work between codes and testing has been done, there are anomalies. The introduction of multiplicative numerical factors into the computations to add conservatism to the design calculations must be justified on physical grounds rather than by an arbitrary factor to envelop conjectured uncertainties. In the case of pressure boundary breeches, mechanical shock and pipe whip effects on desorption and lift-off must be included.

3. The materials to be used and their sensitivity on the transport case must be identified. The particular-vs-generic transport properties of the materials must be evaluated. It has been common in past nuclear systems to replace materials (and add substances to the coolant) because of operational shortfalls or the need for system improvements, and the transport safety case may be sensitive to the (changeable) surface state of component materials. How can one be sure this situation will not occur, or if it does, will the required testing be possible late in the reactor life if the component plays an essential transport role?

4. Once the actual reactor design is available, the transport pathways that result from the accident conditions must be identified along with the relevant models and data needed for the resulting calculations. The actual design will be key to the analysis as the results to date have been focused on a conjectured configuration which may or may not be a good model for planning future work. The identification of margins, sensitivities, and possible mitigating actions by the reactor operator needs to be determined.

5. Technical specifications for the maximum acceptable FP loading of key components must be determined along with practical methods of ensuring that the levels can be determined during normal operation. A recovery plan for handling and recovering from exceeding the limits should be identified.

6. The fuel database must be developed as well as fuel-failure models and fuel material properties (both measurable and process controlled). Past work has identified pyrocarbon as important for the structural stability of the particle and silicon carbide for both strength and as a diffusion barrier.

This list is not meant to be comprehensive; it is to serve as a starting point for the review of the safety case. It is anticipated that much of this information will be supplied as the NGNP design proceeds.

With the above issues in mind, the regulators and reviewers will also need to consider the following (these may be policy issues to be answered at a later date, depending on the course of events):

- How exactly should the defense in depth and enhanced safety be defined and achieved? HTGR FPT as defined to date has replaced the traditional solid barriers and robust filters with complex physical deposition mechanisms that can be sensitive to the local environment. Only limited large-scale testing has been done.

- There is a large amount of past work in the field, both national and international; however, much of the work was conducted under varying quality assurance conditions in a host of countries. Can this body of data be used to minimize the requirements for future testing?

- The designers appear to be calling for a mechanistic source term based on some rather complex physical transport modeling, heavy reliance on fuel performance, modest confinement building performance, and limited emergency planning zones. Can these 
four issues be reconciled with the very limited large-scale experience with this concept and the few mitigation features available for use during an accident?

\subsubsection{Considerations in attempting modeling gap mitigation in the near term}

The traditional FP modeling approach used for gas-cooled reactor accident evaluation has been to model each step in the transport pathway from fuel to the atmosphere for each FP of interest. Although some judgments have been applied as to which FP nuclides and which steps are most urgent to model, paring back somewhat the amount of data and number of required models, this approach has led to a proliferation of models with great demands for data. Moreover, the experiments on which these models are based are generally expensive because (1) radioactive material is usually involved, requiring special facilities, (2) many difficult issues of chemistry and physics are involved, and (3) experimental conditions are often extreme and hence difficult to achieve.

\subsubsection{Considerations regarding the need for gap mitigation}

Because of the above reasons and others, history shows that experiments on which FP models are based are often under-funded, with compromises as to the amount of data taken, with insufficient replication for assessment of accuracy, and compromises as to the attainment of required experimental conditions. This, together with difficult physics such as for particle adhesion, liftoff under vibrating high-flow conditions, and diffusion in a radiation field, has led to the traditionally high-error bars normally seen for FPT models.

Consider the consequence of stringing together perhaps five (or more) such models, from the fuel through the containment, to obtain the release. The result is the typical multidecade error bar. This uncertainty puts a burden on all aspects of proving safety, especially on fuel integrity requirements. This is not meant to discourage the historical approach; rather, it is meant to focus on a systematic approach to testing and data collection. The goal should be to minimize the total transport uncertainty rather than the uncertainty of any particular transport step. In the past, integral experiments have been proposed to examine the predictions made by single effects testing. This global testing can be very valuable to help bound the uncertainty.

The irony is that all indications are that FP releases are quite low even for the most serious accidents, yet development of the numerous individual release models requires great expense and long lead times, and ends up with a predictive method with large uncertainty bands. Adoption of this traditional approach for the NGNP would lead to a financial and lead time burden that perhaps may be avoided by an alternative approach. The regulator should anticipate creative approaches to the NGNP as the traditional approach may burden the project unnecessarily.

\subsubsection{Intermediate- and Long-Term Approaches}

\subsubsection{Gap mitigation by means of integral experiments}

One method for avoiding the need for developing a large number of FPT models is to determine FP release directly from integral experiments. For this method to realistically replace individual modeling, (1) the final chosen materials must be used, that is, prototypic fuel, graphite, and primary system alloys; and (2) an acceptable range of experimental conditions must be employed.

One option to consider is the actual use of a demonstration reactor for at least some of the FP release testing. It is possible that some data could be obtained from operating international test reactors. This approach not only avoids the time and expense of developing numerous FPT models but also has the potential for shrinking the error bars on the release. It is difficult to estimate the value of rendering more precise FP release estimates, but the benefit may be large. Particularly, some development pressure on fuel behavior may be mitigated by relieving the need for designing for the large transport uncertainty. 
This approach is not presented as a certain alternative method but only as an attractive idea that may be explored by a designer. Whether it proves practical or not depends on some close evaluation of the particular details required for integral experimentation and the operating envelope of the test reactors.

\subsubsection{Modeling gap mitigation by using filtered confinement}

Employing an always-filtered confinement with a proven FP capture efficiency does not eliminate the need for conventional FPT modeling. However, it does alleviate the importance of reducing error bars on the release estimates and, consequently, on the expense of the individual experimentation required for developing the models.

To avoid confusion at this point, note that the concept involves at least the following three filter options:

- A reactor building with a vent that opens up only during an over-pressurization event such as a large reactor pipe break; the helium is discharged directly to the environment through the vent. After the pressure surge is over, or for small leaks, the vent is closed and the helium release from the reactor is handled by the building heating, ventilating, and air conditioning (HVAC) system or simply leaks out.

- A filtered/vented reactor building with a vent that opens up during an over-pressurization event such as a large reactor pipe break; the helium is discharged directly to the environment. After the pressure surge is over or for small leaks, the vent closes and any further releases from the reactor are routed through filters prior to release to the environment. This option only requires a modest filter unit.

- An always-filtered reactor building that passes all the reactor cavity effluent through a filter system. Since this filter system must handle the large sudden release from a major primary circuit break, it will be a large robust unit.

The first two options have been proposed as design options; the third is the subject of this section. It is not a trivial addition to the project.

As a hypothetical illustration, suppose that $1 \mathrm{Ci}$ of ${ }^{137} \mathrm{Cs}$ is the desired accident release limit, but model predictions indicate a range from 0.1 to $10 \mathrm{Ci}$. A two-decade uncertainty is not atypical for such an estimate. The situation as it stands could very well already be satisfactory, but because of the modeling uncertainty, some improvement must be made, possibly by higher fuel retention, for reducing the upper-limit bound. This is an illustration of how uncertainty in FPT modeling can lead to increased fuel development costs or demand other mitigation means.

If a $95 \%$ efficient always-filtered confinement (no direct venting under any condition) were used, the very same set of models and accident conditions would predict 0.005 to $0.5 \mathrm{Ci}^{137} \mathrm{Cs}$ release. The effect of the filter is to place the upper bound within specification limits. The filter thus enables satisfactory results with less precise models. Possibly also, the $95 \%$ efficient filter may permit some uncertainty as to the materials (or even the fuels) used for the experimentation, permitting use of historically developed models employing out-of-date materials.

Large filters (sand and fiberglass) have been used by the DOE in radiochemical processing plants, and design improvements in large-scale filters could make them of interest to the NGNP [5-11, 5-23]. Other gas-cleaning devices such as acoustic agglomerators, scrubbers, precipitators, and cyclones have been considered as emergency air-cleaning devices in both once-through and recirculating systems. If dust is truly an issue, filter plugging will need to be addressed. Reference 5-23 discusses many of these filtering, flow, and plugging issues for a sodium-cooled reactor, and while sodium aerosols are considered rather than carbon, the flow, filter plugging, reliability, and size issues are similar. There is considerable literature on gas cleaning devices. They tend to be 
reasonably well understood, and testing is much easier than nuclear system testing. Because they are separate from the nuclear systems, they follow the traditional defense-in-depth philosophy in a straightforward manner and can be tested in isolation. Cost may be an issue, however.

Filters may also impact a containment design. In the unlikely event that the NGNP proceeds with a traditional containment, most of the containment analysis tools will be available since containments have been well studied by LWR designers. However, one new issue with the containment is that helium, unlike steam, is noncondensable, and a means of relieving the pressure in the containment will be necessary. This could be done with some sort of vent filter as has been explored for European LWR reactors [5-20 through 5-22].

A filter system was installed on the Los Alamos Ultra High-Temperature Reactor Experiment (UHTREX), a small gas-cooled test reactor, and a filter system was chosen to handle the effluent under accident conditions [5-24, 5-25]. This is a small-scale system but serves to illustrate a practical application.

A major issue is the impact of the (always in place) filters on the confinement design of the NGNP. If there is a significant pressure increase in the building during accidents because of flow resistance, the building will have to be fortified to withstand the pressure or made larger to lower the pressure, both resulting in cost increases above and beyond the increased cost of the larger filter system. However, these costs will have to be weighed against the program costs of collecting FPT and fuel qualification data, as well as any setbacks that may occur in the process. The magnitude of the design problem may be reduced if a "leak-before-break" assumption is acceptable and if this "leak" is of a manageable size, as the resulting pressure surge could be much smaller with the accompanying reduced demand on the building and filters. These are designer issues rather than regulator issues, but the regulator must anticipate a range of possible solutions at this early stage of NGNP development and be prepared for creative trade-offs.

Considering the above, it may be prudent to examine the following:

- the state of the art in radiological filter design, the potential for large-scale filtering, and the likely filter attenuation required for the NGNP;

- the degree to which FPT uncertainty can be mitigated and compensated for;

- the impact a radiological filter may have on the design and operation of the reactor building;

- the impact of the filter concept on the licensing process, negative interactions with the other parts of plant, and associated requirements for the FPT task; and

- the possible relaxation of reactor system component performance and safety issues.

While the regulator should be prepared to follow the current safety issues based on a vented or a filtered/vented confinement, one should also anticipate creative solutions designed to avoid the difficult and expensive tasks associated with the intricacies of FPT physics and chemistry.

\subsection{SUMMARY}

In considering predicted FPT and releases to the environs in the case of Generation IV reactors such as NGNP, a mitigating factor is that an NGNP major design objective is to employ passive safety features that limit reactor responses in all plausible accident scenarios such that no significant fuel failure and FP releases occur. To the extent that the NGNP design successfully meets this objective, the burden of proof for estimating potential FPT and releases outside the confinement area is lessened. In those postulated events where FPT is an issue, the associated phenomena to be dealt with are complicated. In addition, the coefficients and other parameters in the FPT models often have very large uncertainties. The NGNP program will first have to identify and characterize the materials of interest and the reactor design and safety case before this uncertainty can be reduced, especially if 
a vented confinement option is used. A host of past data, models, and computer codes are available to begin this task, but the sensitivity of the materials to the specific operating environment will limit the amount of past data that can be applied directly to this design. It is likely that a considerable amount of testing would be necessary.

The major FPT phenomena of interest identified in the PIRT exercise [1-1] are well covered in this report. The primary issues noted related to particulars of the confinement design (which would have a major impact on "gap priorities"), are FP releases via normal helium leakage, and the effects of dust-borne FPs and mechanical shock and vibrations during rapid discharge in a D-LOFC accident.

It is believed that through a concerted effort, the required data can be obtained and the necessary models confirmed or developed, leading to a FPT code for the NGNP. Some of this effort is under way for the international projects. It may also be possible to take advantage of operating test reactors to obtain pertinent information. However, at present, these paths are likely to be expensive and timeconsuming, so the designers may look for alternative solutions to this issue to accelerate NGNP development. Thus, the regulator may find it prudent to be prepared to entertain other ideas such as large filters capable of handling the high flow of a depressurization event.

\section{REFERENCES}

5-1. Deep-Burn Modular Helium Reactor Fuel Development Plan, ORNL/TM-2002/135 (GA224-0-TRT-000167), September 2002.

5-2. Verification and Validation Plan for Radionuclide Control Methods, DOE-HTGR88125/Rev. 1, November 1988.

5-3. "Fission Product Transport and Source Term Modeling," Section 2.2.5, INEEL/EXT-0502581, Next Generation Nuclear Plant Research and Development Program Plan, Idaho, Oak Ridge, and Argonne National Laboratories, January 2005.

5-4. J. Van der Merwe, Development and Validation of Fission Product Release Models and Software at PBMR, 2nd International Tropical Meeting on High Temperature Reactor Technology, Bejiing, China, September 23-24, 2004.

5-5. $\quad$ Considerations in the Development of Safety Requirements for Innovative Reactors: Applications to Modular High Temperature Gas Cooled Reactors, IAEA-TECDOC-1366, August 2003.

5-6. R. C. Martin, Compilation of Fuel Performance and Fission Product Transport Models and Database for MHTGR Design, ORNL/NPR-91/6, October 1993.

5-7. R. Bird et al., Transport Phenomena, 2nd Edition, John Wiley \& Sons Inc., New York, 2002.

5-8. $\quad$ D. A. Dilling et al., The Modular High Temperature Gas-Cooled Reactor (MHTGR) Containment Trade Study, ASME/IEEE Joint Power Generation Conference, Boston, MA, October 21-25, 1990.

5-9. D. A. Dilling et al., A Vented Low Pressure Containment Strategy for the Modular High Temperature Gas-Cooled Reactor (MHTGR), GA-A21622, April 1994.

5-10. P. Lobner and R. Zounes, Preliminary Evaluation of the Containment Building for the NPRMHTGR and Consideration of Potential Design Trade-Offs, SAIC-89/1546, 1989.

5-11. G. A. Sehmel, A Review of Potential Alternatives for Air Cleaning at the Hanford Waste Vitrification Plant, PNL-7391, July 1990. 
5-12. I. B. Wall and M. Merilo, Containment Filtration Systems Tests Advanced Containment Experiments (ACE) Project: Summary Report, EPRI-TR-100346, 1992.

5-13. Fuel Performance and Fission Product Behavior in Gas-Cooled Reactors, IAEA-TECDOC978, November 1997.

5-14. M. Perkin, Flow Induced Vibration Problems in Gas-Cooled Reactors in Practical Experiences with Flow-Induced Vibrations, Symposium Karlsruhe/Germany, September 3-6, 1979, University of Karlsruhe, edited by E. Naudascher and D. Rockwell, Springer-Velag, Heildberg, 1980.

5-15. Design of High Temperature Engineering Test Reactor (HTTR), JAERI-1332, September 1994.

5-16. Plateout Phenomena in Direct-Cycle High Temperature Gas-Cooled Reactors, EPRI, Palo Alto, CA, 2002. 1003387.

5-17. TRISO-Coated Particle Fuel Phenomenon Identification and Ranking Tables (PIRTs) for Fission Product Transport Due to Manufacturing, Operations, and Accidents, NUREG/CR6844, July 2004.

5-18. G. Ziskind et al., "Resuspension of Particulates from Surfaces to Turbulent Flows-Review and Analysis," Journal of Aerosol Science 26, 613-644 (1995).

5-19. E. Hontanon et al., "The CAESAR Code for Aerosol Resuspension in Turbulent Pipe Flows. Assessment Against the Storm Experiments," Journal of Aerosol Science 31, 1061-1076 (2000).

5-20. J. L. Kovach, Review of Containment Vent Filter Technology, NUREG/CP-0098 (CONF880822), Vol. 1, Proceedings of the 20th DOE/NRC Nuclear Air Cleaning Conference, Boston, MA, 1988.

5-21. Air Cleaning in Accident Situations, Nuclear Energy Agency, OECD, 1984.

5-22. J. S. Ludwigsen, Investigation of Options for Venting and Filtering of Nuclear Reactor Plants, SAND92-2593, 1993.

5-23. R. K. Hillard et al., Evaluation of Air Cleaning System Concepts for Emergency Use in LMFBR Plants, HEDL-TME-76-41, December 1976.

5-24. J. D. Defield and H. J. Ettinger, Efficiency Testing The Air Cleaning System for a High Temperature Reactor, CONF-680811-2, LA-DC-9483.

5-25. USAEC Report, Ultra High Temperature Reactor Experiment (UHTREX) Facility Description and Safety Analysis Report, LA-3556, 1966.

5-26. J. Wang et al., "TIMCOAT: An Integrated Fuel Performance Model for Coated Particle Fuel," Nuclear Technology 148, 68-96 (2004).

5-27. Manual of Protective Action Guides and Protective Actions for Nuclear Incidents, EPA-400R-92-001, May 1992. 



\section{HIGH-TEMPERATURE MATERIALS}

This section provides background information on development issues for the major structural components and materials, including metallic and some nonmetallic materials, as needed for modular HTGR service (graphite is covered in Sect. 7). The phenomena potentially influencing reactor safety, as identified in the PIRT exercises [1-1], are described and addressed for each of the significant areas, along with information about the phenomena identified as technology gaps. Where gaps are evident, data needs are compared with the available data and analysis tools. Solutions for addressing the gaps are noted where appropriate.

\subsection{MAJOR PHENOMENA OF INTEREST}

The major aspects of materials degradation phenomena that may give rise to regulatory safety concern for the NGNP were evaluated for major structural components and the materials comprising them, including metallic and nonmetallic materials for control rods, other reactor internals, and primary circuit components; metallic alloys for very high-temperature service for heat exchangers and turbomachinery; metallic alloys for high-temperature service for the RPV and other pressure vessels and components in the primary and secondary circuits; and metallic alloys for secondary heat transfer circuits and the BOP. These materials phenomena were primarily evaluated with regard to their potential for contributing to FP release at the site boundary under a variety of event scenarios covering normal operation, anticipated transients, and accidents.

Of all the high-temperature metallic components, the one most likely to be heavily challenged in the NGNP will be the IHX. Its thin internal sections must be able to withstand the stresses associated with thermal loading and pressure drops between the primary and secondary loops under the environments and temperatures of interest. Several important materials-related phenomena related to the IHX were identified, including crack initiation and propagation; the lack of experience of primary boundary-design methodology limitations for new IHX structures; and manufacturing phenomena for new designs.

Specific issues were also identified for RPVs that will likely be too large for shop fabrication and transportation. Validated procedures for on-site welding, post-weld heat treatment (PWHT), and inspections will be required for the materials of construction. High-importance phenomena related to the RPV include crack initiation and subcritical crack growth; field fabrication process control; property control in heavy sections; and the maintenance of high emissivity of the RPV materials over their service lifetime to enable passive heat rejection from the reactor core. All identified phenomena related to the materials of construction for the IHX, RPV, and other components were evaluated and ranked for their potential impact on reactor safety.

The phenomena ranked by the PIRT panel with high importance and low or medium knowledge bases are of the utmost concern and should have the highest priority for research effort. Similarly, phenomena with a medium importance rank and low or medium knowledge base are of concern and should have moderate priority for research effort.

Thus, the following phenomena represent the most significant technology/data gaps based on their high importance ranking with only a low or medium knowledge ranking.

\subsubsection{Phenomena Ranked Importance—High, Knowledge—Low}

\section{RPV Rack Initiation and Subcritical Crack Growth}

The PMR candidate RPV material, 9Cr-1 Mo steel (grade 91), must be assessed for crack growth phenomena due to transients and operationally induced thermal loading, pressure loading, residual 
stress, and the presence of existing flaws (degradation of welds, cyclic loading, low cycle fatigue). There is a limited database from fossil energy applications at NGNP PMR temperatures. Low-cycle fatigue data in air, vacuum, and sodium (Argonne National Laboratory unpublished data) at $482^{\circ} \mathrm{C}$ show life is longest in sodium, followed by vacuum and air. Aging degradation in helium (depending on impurities) will most likely be greater than in air. Aging in impure helium may perhaps depend on impurity type and content [6-1 through 6-4]. Due to limited data at the requisite temperature, the panel rated this phenomenon's knowledge level low.

\section{Compromise of RPV Surface Emissivity Due to Loss of Desired Surface Layer Properties}

To ensure passive safety, high emissivity of the RPV is required to limit RPV and core temperatures. High emissivities must be maintained on both inside and outside surfaces of the RPV. Formation and control of surface layers must be considered under both helium and air environments. There are limited studies on ferritic stainless steels proposed for the PMR RPV and on SA 508 carbon steel proposed for the PBR RPV that show the potential for maintaining high emissivity under expected operational environmental conditions. The panel rated this phenomenon's knowledge level low.

\section{RPV Material Properties Degradation During Field Fabrication}

There are significant questions about the site of the NGNP and whether a factory-fabricated RPV can be shipped to a remote sire for the first unit (proposed to be located in Idaho). Fabrication process control must address field fabrication because of vessel size [including welding, post-weld heat treatment, section thickness (especially with 9Cr-1 Mo steel), and pre-service inspection]. Fossil energy experience indicates that caution must be taken. On-site nuclear vessel fabrication is unprecedented [6-1 through 6-4]. The panel rated this phenomenon's knowledge level low.

\section{RPV Material Property Control in Heavy Sections}

Heavy-section properties are difficult to obtain because of hardenability issues. Adequate large ingot metallurgy technology does not exist for 9Cr-1 Mo steel proposed for the PMR RPV.

Maintaining fracture toughness, micro-structural control, and mechanical properties in the throughwall thickness of heavy sections of $9 \mathrm{Cr}$ ferritic alloy materials must be maintained. Very limited data exist with even less on materials over 3 to 4 in. in section thickness. There is very limited data available for specimens from 300-mm-thick forgings, and these data show thick-section properties lower than thin-section properties [6-1 through 6-4]. The panel rated this phenomenon's knowledge level low.

\section{Aging Fatigue, Environmental Degradation of Insulation Materials}

The major concern is about insulation debris plugging core cooling channels causing fuel damage due to local overheating. The debris would be chunks of internal insulation falling off or being swept off by helium coolant flow; ceramic sleeves or carbon-carbon composites would be the most likely source of such debris. Little system-relevant information about insulation failure mechanisms is available [6-3 through 6-12]. The panel rated this phenomenon's knowledge level low.

\section{IHX Crack Initiation and Propagation}

Environmental effects (creep crack growth, creep, creep-fatigue, aging) must be addressed with regard to subcritical crack growth, subject to impacts of design issues, particularly for thin-section alloy structures. Stresses on the IHX (both thin and thick sections) can lead to these failure phenomena. Thermal transients can cause loss-of-toughness concerns. Carbide redistribution as a function of thermal stress and loading direction can change through-wall-thickness properties. More is known about Inconel 617 from HTGR and industry usage than for Haynes 230. Both environment and creep play significant roles in initiation and cyclic crack growth rate of Inconel 617 and Haynes 230. Mechanistic models for predicting damage development and failure criteria for time-dependent 
phenomena have to be developed to enable conservative extrapolation from short-term laboratory test data to long-term design life [6-3 through 6-12]. The panel rated this phenomenon's knowledge level low.

\section{Primary Boundary Failures in Compact IHX_Role of Design Methods}

Time-dependent design criteria for complex structures need to be developed and verified by structural testing. ASME Code-approved simplified methods have not been proven and are not permitted for compact IHX components. There is no experience for the complex shape IHX nor for designing and operating such high-temperature components in the class 1 environment. Difficulties of design and analyses of compact IHX are discussed in the Refs. 6-3 through 6-12. The panel rated this phenomenon's knowledge level low.

\section{Primary Boundary Failures in Compact IHX_Role of Manufacturing Controls (Such as Joining)}

Compact heat exchanger (CHE) cores (if used) will require advanced machining, forming, and joining (e.g., diffusion bonding, brazing, etc.) methods that may impact component integrity during operation and aging at high-temperature operating conditions. CHEs must be assessed against the option of using traditional tube and shell concepts. However, these phenomena related to degradation and failure are generic and extend beyond the CHEs to all the very high-temperature heat exchangers. The panel rated this phenomenon's knowledge level low.

\section{Primary Boundary Failures in Compact IHX_Role of Inspection/Testing Regime}

Traditional nondestructive examination (NDE) methods will not work for CHEs because of geometrical constraints. Proof-testing of some kind will be required (maybe leak testing with tracer). Pre-service testing will be difficult, and in-service testing will be even more so. Condition monitoring may be useful. Traditional approaches to preoperational testing, pre-service inspection, fitness-for-service tests, and the use of leak tests provide very little knowledge base here. Margins to failure should be determined by some method other than detecting failures after they occur [6-3 through 6-12]. The panel rated this phenomenon's knowledge level low.

\section{Control Rod Insertion Failures-Role of Composites Structural Design Nethods}

Carbon-carbon composites are prime candidates for high-temperature control rod clad but need approved methods of designing, proof testing, model testing, testing standards, design methods, and validation testing. Some code work is being developed by the American Society of Mechanical Engineers (ASME) and the American Society for Testing and Metals (ASTM). Extensive aerospace industry design and usage can be assessed for applicability [6-6 through 6-12]. The panel rated this phenomenon's knowledge level low.

\section{Compromise of In-Vessel Surfaces Emissivities}

To ensure passive cooling and safety during LOFC, a high emissivity value of the core barrel is required to limit core temperatures. High emissivities must be maintained on both inside and outside surfaces. Formation and control of surface layers must be considered under high-temperature helium environments with potential impurities present. Limited studies on austenitic stainless steel and on SA 508 carbon steel show potential for maintaining high emissivity [6-2 through 6-4 and 6-13 through 6-15]. The panel rated this phenomenon's knowledge level low.

\section{Irradiation-Induced Creep of In-Vessel Metallic Structures}

Irradiation creep and dimensional changes particularly for Alloy $800 \mathrm{H}$ at moderately low dose should be assessed. Alloy 800 is a primary candidate material for both the core barrel and core support floor, as well as control rod cladding. Little information on irradiation creep is available for Alloy $800 \mathrm{H}$ [6-2 through 6-4 and 6-13 through 6-15]. The panel rated this phenomenon's knowledge level low. 


\section{Core Radial Restraint Failure-Role of Composites Structural Design and Fabrication Methods}

Carbon-carbon composites are prime candidates for core restraint structures but need approved design methods. The needs include methods for proof testing, model testing, testing standards, validation tests, handling of scalability issues and fabrication issues, and probabilistic methods of design. Applications in large-scale (meters in diameter) structures, as well as smaller ones, must be addressed. Extensive experience exists within the aerospace industry, but applicability must be assessed [6-6 through 6-12]. The panel rated this phenomenon's knowledge level low.

\section{Environmental and Irradiation Degradation and Thermal Stability of Fibrous Insulation}

Relatively low dose and exposure is expected, but LOFC can result in temperatures high enough to challenge stability of fibrous insulation such as Kaowool. There is a need to assess candidate materials for the effects on micro-structural stability and thermo-physical properties during irradiation and high-temperature exposure in impure helium. Limited commercial information is available for conditions of interest [6-6 through 6-12]. The panel rated this phenomenon's knowledge level low.

\section{Isolation Valve Failure}

Isolation valve failure (including categories such as self-welding, galling, seizing) is possible. Concerns about isolation valves are similar to "breach to secondary" issues on IHX because they would provide barriers to secondary heat transport system. Information is possibly available from previously constructed HTGRs, but relevance of any such information needs to be assessed. State of knowledge about helium-leak-tightness in large valves is unknown [6-2 through 6-4]. The panel rated this phenomenon's knowledge level low.

\section{Other Valve Failures}

Concerns about a variety of valve failure mechanisms that will be design dependent include categories such as self-welding, galling, and seizing. These valves and their materials will need to be assessed once design-specific details are available. Helium tribology issues must be considered. Allowable identified and unidentified coolant leakage rates must be established. Information may be available from previously constructed HTGRs, but relevance needs to be assessed [6-2 through 6-4]. The panel rated this phenomenon's knowledge level low.

\subsubsection{Phenomena Ranked Importance-High, Knowledge-Medium \\ RPV Thermal Aging (Long Term)}

For the PMR, the requirement for a 60 -year lifetime requires that the uncertainty be reduced in the properties of $9 \mathrm{Cr}-1 \mathrm{Mo}$ steel (grade 91), especially for the degradation and aging of base metals and welds for a critical component such as the RPV. It is assumed that Grade 91 is the prime candidate for the PMR as the NGNP, and no back-up material has been proposed nor is considered here for the candidate PMR without active RPV wall cooling. This is beyond the experience base for conditions of interest, extensive fossil energy experience, and code usage although significant aging data exist at high temperatures $\left(500^{\circ} \mathrm{C}\right)$. The need is for long-term aging data at NGNP-relevant temperatures [6-1 through 6-4]. Therefore, the panel rated this phenomenon's knowledge level as medium.

\subsection{PHYSICAL AND SUPPORTING MODEL ASPECTS}

Physical aspects for modeling high-temperature metallic components include the following:

- inelastic materials behavior for materials, times, and temperatures for very hightemperature structures (e.g., creep, fatigue, creep-fatigue); 
- adequacy and applicability of current ASME Code allowables with respect to service times and temperatures for operational stresses;

- adequacy and applicability of current state of high-temperature design methodology (e.g., constitutive models, complex loading, failure criteria, flaw assessment methods);

- effects of product form and section thickness;

- joining methods including welding, diffusion bonding, and issues associated with dissimilar materials in structural components;

- effects of irradiation on materials strength, ductility, and toughness;

- degradation mechanisms and inspectability;

- oxidation, carburization, decarburization, and nitriding of metallic components in impure helium and helium-nitrogen;

- micro-structural stability during long-term aging in environment;

- effects of short and long term on mechanical properties (e.g., tensile, fatigue, creep, creep-fatigue, ductility, toughness);

- high-velocity erosion/corrosion;

- rapid oxidation of graphite and carbon-carbon composites during air-ingress accidents;

- compatibility with heat-transfer media and reactants for hydrogen generation; and

- development and stability of surface layers on RPV and core barrel affecting emissivity.

Physical aspects for designing and modeling high-temperature structural composites (such as carbon-carbon or silicon carbide-silicon carbide) include the following:

- effects of composite component selection and infiltration method;

- effects of architecture and weave;

- materials properties up to and including very high temperatures (e.g., strength, fracture, creep, corrosion, thermal shock resistance);

- effects of irradiation on materials strength and dimensional stability;

- fabrication scaling processes;

- adequacy and validation of design methods; and

- degradation mechanisms and inspectability.

\subsection{CLOSING THE GAPS-STAGES OF MODEL DEVELOPMENT}

\subsubsection{Near Term}

It is necessary to initiate as soon as possible development of the data and models needed by ASME Boiler and Pressure Vessel (B\&PV) Code Subcommittees to formulate time-dependent failure criteria that will ensure adequate life and safety for metallic materials in the NGNP. These include obtaining the data necessary to develop experimentally based constitutive models for the NGNP construction materials. Those models are the foundation of the inelastic design analyses specifically required by ASME B\&PV Sect. III Division I Subsection NH. Safety assessments, dependent on time-dependent flaw growth and the resulting leak rates from postulated pressure-boundary breaks, will require a flaw assessment procedure capable of reliably predicting crack-induced failures as well as the size and growth of the resulting opening in the pressure boundary.

Additionally, materials data and extrapolation procedures must be developed and guidance provided to ensure that allowable operation period and range of stress and temperature for materials 
of construction are extended to meet the proposed operating temperatures and lifetimes. Creepfatigue rules are an area of particular concern for the materials and temperatures of interest and must be updated and validated. Since IHX sections must operate at the full exit temperature of the reactor, effort should be initiated to obtain data supporting the determination of the metallurgical stability and environmental resistance of IHX materials in anticipated impure helium coolant environments for the lifetimes anticipated. Several materials-related phenomena related to the IHX were identified as having a high importance for potentially contributing to FP release at the site boundary and a low level of knowledge with which to assess their contribution to such a release. Therefore, work should be initiated early to quantify crack initiation and propagation (due to creep crack growth, creep, creep-fatigue, and aging).

\subsubsection{Intermediate and Long Term}

Specific issues must be addressed for RPVs that are too large for shop fabrication and transportation. Validated procedures for on-site welding, PWHT, and inspections must be developed for the materials of construction. For vessels using materials other than those typical of LWR construction to enable operation at higher temperatures, confirmation of their fabricability (especially, effects of forging size and weldability) and data on their irradiation resistance is needed. Three materials-related phenomena related to the RPV fabrication and operation were identified as having a high importance for potentially contributing to FP release at the site boundary and a low level of knowledge with which to assess their contribution to such a release, particularly for $9 \mathrm{Cr}-1 \mathrm{Mo}-\mathrm{V}$ steels capable of higher-temperature operation than LWR vessel carbon steels. Aspects needing attention include crack initiation and subcritical crack growth, field fabrication process control, and property control in heavy sections.

In addition, for high-temperature metals technology, there is a need for analytical models, in particular for developing time-dependent design criteria for complex structures, along with verification by structural testing. ASME Code-approved simplified methods have not yet been proven and are not permitted for compact IHX components.

Analytical modeling of carbon-carbon composite behavior would be useful in developing approved methods for designing, proof testing, model standard testing, validation tests, and probabilistic methods of design. Scalability and fabrication issues must be addressed. Large-scale (meters in diameter) structures as well as smaller ones must be covered.

\subsection{SUMMARY}

Technology gaps for NGNP reactor materials (excluding graphite), which exist in the following areas, correspond directly to those identified by the PIRT panel:

- RPV crack initiation and subcritical crack growth,

- RPV surface emissivity and loss of desired surface layer properties,

- RPV material properties degradation during field fabrication,

- RPV material property control in heavy sections,

- aging fatigue and environmental degradation of insulation materials,

- IHX crack initiation and propagation,

- structural design methods of control rod composites,

- in-vessel surface emissivities,

- irradiation-induced creep in in-vessel metallic structures,

- composites structural design and fabrication methods for radial restraint structures, 
- environmental and irradiation degradation and thermal stability of fibrous insulation, and

- valve failure mechanisms for high-temperature conditions.

\section{REFERENCES}

6-1. G. O. Hayner, R. L. Bratton, R. N. Wright, W. E. Windes, T. C. Totemeier, K. A. Moore, W. R. Corwin. T. D. Burchell, J. W. Klett, R. K. Nanstad, L. L. Snead, Y. Katoh, P. L. Rittenhouse, R. W. Swindeman, D. F. Wilson, T. E. McGreevy, and W. Ren, Next Generation Nuclear Plant Materials Research and Development Program Plan, U.S. Department of Energy Generation IV Nuclear Reactor Program, Office of Nuclear Energy Science and Technology, DOE, INL/EXT-05-00758 (Revision 2), September 2005.

6-2. Preliminary Safety Evaluation Report (PSER) for the PRISM LMR, NUREG-1368.

6-3. C. R. Brinkman et al., Development of Stress-Rupture Reduction Factors for Weldments, and the Influence of Pretest Thermal Aging to $50,000 \mathrm{~h}$ on Stress-Rupture and Microstructural Properties of Modified 9Cr-1Mo Steel, ORNL/9Cr/90-1, February 1990.

6-4. ASME Boiler and Pressure Vessel Code, Code Case N-499.

6-5. J. M. Corum and J. J. Blass, "Rules for Design of Alloy 617 Nuclear Components to Very High Temperatures,: PVP 1991 Fatigue, Fracture and Risk, San Diego, CA, 1991.

6-6. ASME Boiler and Pressure Vessel Code, Section III, Subsection NH.

6-7. ASME Boiler and Pressure Vessel Code, Code Case N-201.

6-8. R. I. Jetter, "Subsection NH-Class 1 Components in Elevated Temperature Service," in Companion Guide to the ASME Boiler and Pressure Vessel Code, 2002.

6-9. $\quad$ R5, Assessment Procedure for the High Temperature Response of Structures (1999).

6-10. R. A. Ainsworth, "R5 Procedures for Assessing Structural Integrity of Components Under Creep and Creep-Fatigue Conditions," Int. Mater. Rev., 51(2), 107 (2006).

6-11. D. S. Griffin, "Elevated-Temperature Structural Design Evaluation Issues in LMFBR Licensing," Nucl. Eng. Des., 90, 299-306 (1985).

6-12. R. L. Huddleston and R. W. Swindeman, Materials and Design Bases Issues in ASME Code Case N-47, ORNL/TM-12266 (NUREG/CR-5955), Oak Ridge National Laboratory, April 1993.

6-13. R. L. Klueh, Elevated-Temperature Ferritic and Martensitic Steels and Their Application to Future Nuclear Reactors, ORNL/TM-2004/176, Oak Ridge National Laboratory, November 2004.

6-14. T. McGreevy and R. I. Jetter, "DOE-ASME Generation IV Materials Tasks," PVP2006ICPVT-11 2006 ASME Pressure Vessels and Piping Division Conference, July 23, 2006.

6-15. K. Natesan et al., Materials Behavior in HTGR Environments, NUREG/CR-6824 (ANL02/37), July 2003. 



\section{GRAPHITE}

Background information on graphite development and testing issues for the core, reflectors, and major structural components are provided in this section. Also noted is how this material is to be adapted to the special very high-temperature and irradiation conditions peculiar to modular HTGR service (other high-temperature materials are covered in Sect. 6). The phenomena influencing reactor safety (from the PIRT exercises) are described and addressed for each of the significant areas. When gaps are noted, data needs are compared with available data and analysis tools. Solutions for addressing the gaps are noted where appropriate. More information about the phenomena identified as technology gaps is found in the report of the results of the PIRT process [1-1].

\subsection{MAJOR PHENOMENA OF INTEREST}

For graphite, the most significant technology and data gaps relate to the lack of confirmatory data for the grades of graphite selected by potential NGNP vendors. This situation has occurred because the graphite grades used in prior HTGRs are no longer available, and thus development of new grades has been required. Other data gaps identified relate to the increased temperature of the NGNP compared to prior graphite-moderated reactors, or, in the case of the PBR, the larger neutron dose that the core components will experience compared to that of previous HTGRs licensed in the United States.

Another gap is related to the lack of consensus codes and standards. Efforts are under way through the ASME to develop a consensus design code for graphite core components, but to date a useable code has not been approved. ASTM test standards exist for many of the physical properties of concern to the reactor designer, but further work is required, especially in the area of small (irradiation) specimen test methods.

The phenomena ranked by the PIRT panel with high importance and low or medium knowledge bases are of the utmost concern and should have the highest priority for research effort. Similarly, phenomena with a medium importance rank and low or medium knowledge base are of concern and should have moderate priority for research effort.

Thus, the following phenomena represent the most significant technology/data gaps for graphite based on their high importance ranking with only a low or medium knowledge ranking.

\subsubsection{Phenomena Ranked Importance—High, Knowledge-Low}

\section{Irradiation-Induced Creep (Irradiation-Induced Dimensional Change under Stress)}

Stress due to differential thermal strain and differential irradiation-induced dimensional changes would very quickly cause fracture in the graphite components if it were not for the stress relief due to irradiation-induced creep. The phenomena and mechanism of irradiation-induced creep in graphite is therefore of high importance. Currently, there are no creep data for the graphite grades being proposed for use in the NGNP. However, creep at low dose follows a linear law, which can be explained through a dislocation pinning/unpinning model from Kelly and Foreman [7-1]. Marked

deviation from this law has been observed at intermediate neutron doses. The applicability of this law has been extended by taking into account changes in the pore structure that manifest themselves as changes in the coefficient of thermal expansion (CTE) with creep strain [7-2]. However, the current creep law breaks down at high-temperature, moderate-dose and moderate-temperature, high-dose combinations. A new model for creep is needed which can account for the observed deviations from linearity of the creep strain rate with neutron dose. Existing and new models need to be shown to be applicable to the currently proposed graphite grades. Knowledge rank was therefore considered low. 


\section{Irradiation-Induced Change in CTE, including the Effects of Creep Strain}

Differential thermal strains occur in graphite components due to temperature gradients and local variation in the CTE. The CTE variations are dependent upon the irradiation conditions (temperature and dose) and the irradiation-induced creep strain [7-2 through 7-5]. Thus the importance ranking is high for this phenomenon. Irradiation-induced changes in CTE are understood to be related to changes in the oriented porosity in the graphite structure. These changes are observed to be different when graphite is placed under load (stress) during irradiation. The direction and magnitude of the stress (and the creep strain) affect the extent of the CTE change. There are insufficient data available for the effect of creep strain on CTE in graphite. Moreover, none of the available data are for the grades proposed for the NGNP. Thus the knowledge rank is low.

\section{Irradiation-Induced Changes in Mechanical Properties (Strength, Toughness), Including the Effect of Creep Strain (Stress)}

Graphite properties are known to change with neutron irradiation, the extent of which is a function of the neutron dose, irradiation temperature, and irradiation-induced creep strain. Local differences in moduli, strength, and toughness due to neutron fluence and temperature gradients must be accounted for in design. The importance of this phenomenon is thus ranked high. Although data exist for the effect of neutron dose and temperature on the mechanical properties of graphite, there are insufficient data on the effects of creep strain on the mechanical properties. Moreover, none of the available data are for the grades currently being considered for the NGNP. Knowledge ranking is therefore low.

\section{Blockage of Coolant Channel in a Fuel Element Block or a Reactivity Control Block Due to Graphite Failure and/or Graphite Spalling.}

Significant uncertainty exists as to the stress state of any graphite component in the core. Moreover, the strength of the components changes with dose, temperature, and creep strain. The combination of these factors makes the probability of local failure, graphite spalling, and possible blockage of a coolant channel in a fuel element block or a reactivity control block difficult to determine. Consequently the panel rated this phenomenon's importance as high. Although the changes in properties of graphite have been studied for many years, there are still data gaps that make whole-core modeling very difficult (e.g., effect of creep strain on properties). Moreover, data on the grades selected for NGNP are not available. Therefore, the panel rated the knowledge base for this phenomenon as low. A related concern would be for a blockage that interferes with the ability to insert control or safety rods. In this case the panel rated the knowledge base as medium.

\subsubsection{Phenomena Ranked Importance-High, Knowledge-Medium}

\section{Statistical Variation of Nonirradiated Properties}

The graphite single crystal is highly anisotropic due to the nature of its bonding (strong covalent bonds between the carbon atoms in the basal plane and weak van der Waals bonds between basal planes). This anisotropy is transferred to the filler coke particles and also to the crystalline regions converted by graphitization in the binder phase. Thus, the mechanical and physical properties of graphite vary within a billet due to texture introduced during forming and thermal processing (graphitization). Moreover, there is a statistical variability in the properties between billets within the same lots, between lots, and between batches due to variations in raw materials, formulations, and processing conditions. Therefore it is necessary to develop a statistical data base of the properties for a given graphite grade. The variations in chemical properties (chemical purity level) will have implications for chemical attack, degradation, and decommissioning). Probabilistic design approaches are best suited to capturing the variability of graphite. The panel rated the importance of this phenomenon high. Although other nuclear graphites have been characterized and full databases 
developed, allowing an understanding to be established of the textural variations, only limited data exists on the graphites proposed for the NGNP. Therefore the panel rated this phenomenon's knowledge level as medium.

\section{Consistency in Graphite Quality Over the Lifetime of the Reactor Fleet (for Replacement as an Example)}

Graphite is manufactured from cokes and pitches derived from naturally occurring organic sources such as oil and coal (in the form of coal tar pitch). These sources are subject to geological variations and depletion, requiring the substitution of alternate sources. Therefore, the consistency of graphite quality and properties over the lifetime of a reactor, or the reactor fleet (for replacement, for example), is of concern. The panel ranked the importance of this phenomenon as high. Our understanding of this phenomenon is sufficient to develop generic specifications [ASTM DO2.F, D 7219-05] which should ensure quality and repeatability. However, this has not been proven, especially due to the lack of irradiated properties data. The panel assessed the knowledge base for this phenomenon as medium.

\section{Irradiation-Induced Dimensional Change}

Neutron irradiation causes dimensional changes in graphites. These changes are the result of anisotropic crystal dimensional change rates (a-axis shrinkage and c-axis growth), the interaction of crystal dimensional change with porosity, and the generation of new voids and porosity. The amount of irradiation-induced dimensional change is a function of the neutron dose and irradiation temperature. Consequently, gradients in temperature or neutron dose will introduce differential dimensional changes (strains). Irradiation-induced dimensional changes are the largest source of "internal stress," as opposed to the external load that is applied due to service conditions). Because of the significance of dimensional changes in generating core stresses, the panel gave this phenomenon high importance. Irradiation-induced dimensional changes have been researched for many years, and several dimensional change models have been proposed. However, there is a paucity of data for the dimensional changes of the graphites proposed for the NGNP. Therefore, the knowledge rank was considered medium.

\section{Irradiation-Induced Thermal Conductivity Change}

Displacement damage caused by neutron irradiation introduces additional phonon scattering sites to the graphite crystal lattice and consequently reduces the thermal conductivity. The nature of the irradiation-induced damage is sensitive to the temperature of irradiation. Consequently, the extent of degradation is temperature dependent. In addition, phonon-phonon (Umklapp) scattering increases as the measurement temperature increases and, thus, the thermal conductivity decreases as the temperature increases. At very high irradiation doses, thermal conductivity decreases further, at an increased rate, attributed to porosity generation due to large crystal dimensional change. The thermal conductivity is also subject to some recovery (annealing) on heating above the irradiation temperature (such as during an accident thermal transient). The exact thermal conductivity under all core conditions is therefore subject to some uncertainty. Thermal conductivities lower than that required by the design basis for licensing-basis-event heat removal due to (a) inadequate database to support design over the component lifetime or (b) statistical and textural variations in characteristics of graphites from lot-to-lot have the potential to allow fuel design temperature limits to be exceeded during licensing basis events. The importance of this phenomenon was therefore considered high. Irradiation-induced thermal conductivity changes have been researched for many years, and several conductivity change models have been proposed. However, there is a paucity of data for the conductivity changes of the graphites proposed for the NGNP. Therefore the knowledge rank was considered medium. 


\section{Irradiation-Induced Changes in Elastic Constants, Including the Effects of Creep Strain}

Neutron irradiation induces changes in the elastic constants of graphite. Initial increases in the moduli are attributed to an increase in dislocation pinning points in the basal plane, which reduce the crystal shear compliance, $\mathrm{C}_{44}$. Subsequent changes in the elastic moduli are attributed to porestructure changes (initial pore closures followed by pore generation). Although the understanding of irradiation-induced moduli changes is well developed, there are no direct microstructural observations or sufficiently well-developed models of these mechanisms. Therefore, the knowledge rank was considered medium.

\section{Tribology of Graphite in (Impure) Helium Environments}

Graphite is a naturally lubricious material. However, its behavior can be modified by the helium environment of the NGNP. The abrasion of graphite blocks on one another or of the fuel pebbles on the graphite moderator blocks could produce graphite dust. Studies are needed to assess the effect of the helium environment on the friction and wear behavior of graphite. The possibility that fuel balls can "stick together" and cause a fuel-flow blockage must be explored, although German pebble-bed experience was positive in this regard (i.e., no significant blockages). The consequences of dust generation (as a possible FPT mechanism) and possible fuel ball interactions resulted in the panel ranking the importance of this phenomenon as high. The limited literature on this subject is primarily from the past German program. Consequently the panel ranked the knowledge level as medium.

\section{Degradation of Thermal Conductivity}

The degradation of thermal conductivity in graphite components has implications for fuel temperature limits during loss-of-forced-cooling accidents.

\subsection{PHYSICAL AND SUPPORTING MODEL ASPECTS}

Theoretical models for the effects of neutron damage on the properties of graphite have been developed. However, these models need modification for the new graphites and will need to be extended to higher temperatures and/or higher neutron doses. V\&V of theoretical models can only come through the generation of experimental data on the effect of neutron irradiation on properties. Experimental data to fill the data gaps must be generated in a technology development program. The biggest gaps are related to predicting the buildup in stress in graphite core components. Uncertainties in the temperature and dose received by a component; the severity of temperature and dose gradients in a component; the rate of dimensional change in the specific graphite used in a given design; the extent to which stresses are relieved by irradiation-induced creep; and the extent of changes in key physical properties such as elastic moduli, thermal conductivity, coefficient of thermal expansion, etc., all compound to make the prediction of component stress levels, and hence decisions regarding component lifetime and replacement schedules, very imprecise.

\subsection{CLOSING THE GAPS-STAGES OF MODEL DEVELOPMENT}

\subsubsection{Near Term}

Confirmatory data are needed to show that new graphites behave in a similar fashion to the older graphites upon which the current understanding and knowledge is based. Given the similarity, existing models can be applied to the new graphite grades.

\subsubsection{Intermediate and Long Term}

Current models need to be extended to higher temperatures and neutron irradiation dose as required for NGNP designs. This effort includes improving the existing graphite creep model, which has been shown to break down at large neutron doses (beyond volume turnaround). 
Additionally, whole-core models are required that can predict the stress states of graphite components within the core. Such models should be capable of taking inputs such as temperature and neutron dose and calculating the dimensional change, creep, thermal conductivity, etc., from established theoretical models. Reliable stress-state predictions as a function of reactor life would enable reactor operators and regulators to provide NDE guidance and make decisions regarding inspection intervals and core block replacement.

Basic research should be conducted to strengthen the understanding and modeling capability of the displacement damage process in graphite. Many theoretical models are based on assumptions regarding the graphite lattice defect (vacancy) structure that develops upon irradiation. The nature of the defect structure is known to be temperature dependent and has a marked effect upon the irradiation properties of graphite. Advances in the resolution of electron microscopes and development in ab initio predictions allow molecular-dynamics simulations of the defective structure of irradiated graphite. These simulations are the starting point for new multiscale models of graphite structure that could predict property changes on irradiation in graphite. In addition, in graphite technology, there is a need for analytical models. These models will be developed as part of the NGNP program. The models will be for oxidation, changes in physical properties, irradiation induced dimensional change, and irradiation creep. They will feed into a structural integrity model (code) for the graphite core which will be used for core design and safety assessment.

\subsection{SUMMARY}

Technology gaps for graphite, which exist in the following areas, correspond directly to those identified by the PIRT panel:

- graphite supply (coke sources, graphite vendors);

- confirmatory data for new grades being considered for the NGNP;

- irradiation creep data and effect of creep on properties of candidate NGNP graphites;

- consensus design codes and materials testing standards;

- extension of current theoretical models to higher doses and temperatures;

- development of improved understanding and models for neutron irradiation induced displacement damage in graphite;

- development of whole-core structural models;

- NDE methods for use in and out of core; and

- graphite analytical models for oxidation, property changes, and dimensional changes and creep induced by irradiation.

\section{REFERENCES}

7-1. B. T. Kelly and A. J. E. Foremen, Carbon, 12, 151-158, 1974.

7-2. B. T. Kelly and T. D. Burchell, "The Analysis of Irradiation Creep Experiments on Nuclear Reactor Graphite, Carbon, 32(1), 119-125 (July 9, 1993).

7-3. W. J. Gray, "Constant Stress Irradiation-Induced Compressive Creep of Graphite at High Fluences," Carbon, 11, 383-392 (1973).

7-4. B. T. Kelly, "Irradiation Creep in Graphite-Some New Considerations and Observations," Carbon, 30(3), 379-383 (1992). 
7-5 B. T. Kelly and J. E. Brocklehurst, "UKAEA Reactor Group of Irradiation-Induced Creep in Graphite," Journal of Nuclear Materials 65, 79-85 (1977). 


\section{PROCESS HEAT}

The gap analysis for the process heat sector suffers from a major information gap, that being that the process heat sector of NGNP has been defined only as a means of producing hydrogen, employing $\sim 10 \%$ of the reactor thermal power. No specific chemical process had been defined for hydrogen production. However, candidate hydrogen production processes with potential safety implications were defined in the PIRT process, and background information on these processes is provided in this section. The phenomena influencing reactor (as opposed to process heat plant) safety are described and addressed for each of the significant areas selected. Solutions for addressing the gaps are noted where appropriate. More information about the phenomena identified as technology gaps is found in the report of the results of the PIRT process [8-1].

\subsection{MAJOR PHENOMENA OF INTEREST}

The events and the associated phenomena of high importance identified in the PIRT include the following.

- Cold oxygen $\left(\mathrm{O}_{2}\right)$ and other heavy-gas accidental releases from the process plant that can flow from the chemical plant to the nuclear plant (depending upon wind, relative plant elevations, and nuclear plant air intakes) and potentially impact the integrity of reactor systems, structures, and components (SSCs). All of the proposed processes for production of hydrogen start with water, and thus all of the processes will produce oxygen as a byproduct of hydrogen production. Oxygen is the one common chemical safety issue that can impact nuclear plant safety. At high oxygen concentrations, many "noncombustible" materials become combustible and the potential for spontaneous combustion increases. Increased oxygen levels at the reactor can compromise the functioning of safety equipment. The knowledge level for these phenomena is medium based on the uncertainty in the maturity of the designs to allow complete assessment of vulnerabilities.

- Failure of the IHX leading to potential damage to safety-related SSCs in the reactor due to blow-down effects from large mass transfer and over-pressurization of either secondary or primary side. The knowledge level for these phenomena is medium based on the uncertainty in the maturity of the designs to allow complete assessment of vulnerabilities. The impact of the IHX failure depends upon the selection of the heat transfer fluid in the secondary heat transport loop. Helium is the leading candidate for the heat transport loop, but no final decisions have been made. If helium is used, the helium inventory in the secondary loop may be greater than the inventory in the reactor; thus, any leak in the IHX can significantly increase the total helium inventory involved in any reactor depressurization event.

- Failure of the process heat exchanger (PHX) leading to potential damage to safety-related SSCs in the reactor due to fuel and primary system corrosion from the introduction of corrosive process plant chemicals leaking down the process heat transport line and failing the IHX. The knowledge level for these phenomena is medium based on the uncertainty in the maturity of the designs to allow complete assessment of vulnerabilities.

- Steam generator failures leading to the introduction of steam/water into the primary system, potentially causing a reactivity spike and chemical attack of the TRISO fuel particle coatings and graphite. The knowledge level for these phenomena is medium based on the uncertainty in the maturity of the designs to allow complete assessment of vulnerabilities. Some hydrogen production processes, such as high-temperature electrolysis, require steam as a process feedstock; thus, the high-temperature reactor may be required to provide high-temperature steam. 
- Loss of the pressurized coolant inventory from the intermediate loop leading to a loss of primary reactor heat sink and the potential for hydrodynamic forces on the IHX leading to IHX failure and loss of reactor primary system coolant. The knowledge level for these phenomena is medium based on the uncertainty in the maturity of the designs to allow complete assessment of vulnerabilities.

The PIRT did not rate phenomena associated hydrogen releases as of high importance. Industrial experience, experiments, and theory indicate that hydrogen leaks and accidents have limited impacts beyond the chemical plant because of the extremely high buoyancy and diffusivity of hydrogen.

\subsection{EXPERIMENTAL AND ANALYTICAL MODELING OF PHENOMENA}

The design of the reactor, the process plant, and the intermediate loop needs to consider the events and phenomena of high importance as listed above, but the need for experiments or analytical tools will depend on the hydrogen production processes that are selected and design details. In most cases, proper civil and chemical engineering of the two plants and the intermediate loop can be used to eliminate, minimize, or mitigate the possibility of challenging accidents. Distance, relative elevation, and the selection of noncorrosive, nonhydrogenous intermediate loop working fluid with fast-acting, passive (reverse flow) isolation valves can mitigate the potential for most of the phenomena cited above. The one area that may require further assessment, independent of the specific hydrogen production process, is the release of the by-product oxygen.

\subsection{CLOSING THE GAPS—STAGES OF MODEL DEVELOPMENT}

No specific plant model development is required at this time beyond the application of reasonable engineering judgment, tools, and lessons-learned from chemical plant experience to eliminate or minimize the possibility of accidents affecting both the reactor and the process heat plant.

\subsection{SUMMARY}

Potential phenomena resulting from process plant interface with the reactor are highly design dependent. As was also concluded in the PIRT study [8-1], the design selections should be guided to eliminate or minimize the phenomena that can adversely affect reactor safety.

\section{REFERENCE}

8-1. S. J. Ball and S. E. Fisher, Next Generation Nuclear Plant Phenomena Identification and Ranking Tables (PIRTs)_Volume 1: Main Report, NUREG/CR-6944, Vol. 1 (ORNL/TM2007/147, Vol. 1), Oak Ridge National Laboratory, March 2008 (additional details on process heat applications are found in Vol. 6). 
ORNL/TM-2007/228

\section{INTERNAL DISTRIBUTION}

1. S. J. Ball, 3500, MS-6010

2. T. D. Burchell, 4508, MS-6088

3. W. R. Corwin, 4500S, MS-6161

4. L. B. Dockery, 5700, MS-6165

5. S. E. Fisher, 5300, MS-6050

6. J. C. Gehin, 5700, MS-6172

7. S. R. Greene, 5700, MS-6162

8. D. T. Ingersoll, 5700 , MS-6162
9. G. T. Mays, 5700, MS-6165

10. R. N. Morris, 3525, MS-6295

11. C. V. Parks, 5700, MS-6170

12. J.-P. Renier, 5700, MS-6172

13. J. E. Rushton, 5700, MS-6152

14. J. J. Simpson, 5700, MS-6162

15. S. Zinkle, 4500S, MS-6132

\section{EXTERNAL DISTRIBUTION}

16. S. Bahadur, Office of Nuclear Regulatory Research, MS-9 F39, U.S. Nuclear Regulatory Commission, 11545 Rockville Pike, Rockville, MD 20852-2738.

17. R. Ballinger, Massachusetts Institute of Technology, 185 Albany Street, NW22-117, Cambridge, MA 02139.

18. J. A. Barr, Office of Nuclear Regulatory Research, MS-T10 K8, U.S. Nuclear Regulatory Commission, 11545 Rockville Pike, Rockville, MD 20852-2738.

19. S. Basu, Office of Nuclear Regulatory Research, MS-10 K8, U.S. Nuclear Regulatory Commission, 11545 Rockville Pike, Rockville, MD 20852-2738.

20. R. Bratton, Idaho National Laboratory, P. O. Box 1625, MS-3710, Idaho Falls, ID 83415-3710.

21. G. Brinkmann, AREVA, Framatome ANP GMBH/NGPS3, Freyeslebenstrasse 1, Erlangen, Germany

22. D. E. Carlson, Office of New Reactors, MS-T10 K8, U.S. Nuclear Regulatory Commission, 11545 Rockville Pike, Rockville, MD 20852-2738.

23. P. Cochran, Office of Nuclear Regulatory Research, MS-T10 K8, U.S. Nuclear Regulatory Commission, 11545 Rockville Pike, Rockville, MD 20852-2738.

24. T. L. Cook, NE-33/Germantown Building, U.S. Department of Energy, 1000 Independence Ave., S.W., Washington, DC. 20585-1290.

25. M. Corradini, University of Wisconsin, 1500 Engineering Dr., Madison WI 53706.

26. F. Eltawila, Office of Nuclear Regulatory Research, MS-10 E32, U.S. Nuclear Regulatory Commission, 11545 Rockville Pike, Rockville, MD 20852-2738.

27. M. A. Feltus, NE-33/Germantown Building, U.S. Department of Energy, 1000 Independence Ave., S.W., Washington, DC. 20585-1290.

28. C. W. Forsberg, Massachusetts Institute of Technology, 77 Massachusetts Ave., 24-207a, Cambridge, MA 02139.

29. D. Forsyth, Office of Nuclear Regulatory Research, MS-T10 K8, U.S. Nuclear Regulatory Commission, 11545 Rockville Pike, Rockville, MD 20852-2738.

30. R. Gauntt, Sandia National Laboratories, P.O. Box 5800, MS-0748, Albuquerque NM, 871850748.

31. G. E. Gears, NE-31/Germantown Building, U.S. Department of Energy, 1000 Independence Ave., S.W., Washington, DC. 20585-1290.

32. G. Geffraye, CEA Grenoble DEN/DER/SSTH, 17 rue des Martyrs, 38054 Grenoble Cedex 9, France. 
33. M. B. Gorensek, Computational and Statistical Science Department, Savannah River National Laboratory, 773-42A, Room 136, Aiken, SC 29808.

34. Y. A. Hassan, Department of Nuclear Engineering, Texas A\&M University, Zachry 129, MS3133, College Station, TX 77843-3133.

35. J. S. Herring, Idaho National Laboratory, P.O. Box 1625, MS-3860, Idaho Falls, ID 834153860.

36. M. R. Holbrook, Idaho National Laboratory, P.O. Box 1625, Idaho Falls, ID 83415.

37. N. H. Hudson, Office of Nuclear Regulatory Research, MS-T10 K8, U.S. Nuclear Regulatory Commission, 11545 Rockville Pike, Rockville, MD 20852-2738.

38. A. B. Hull, Office of Nuclear Regulatory Research, MS-T10 M5, U.S. Nuclear Regulatory Commission, 11545 Rockville Pike, Rockville, MD 20852-2738.

39. M. R. Johnson, Office of New Reactors, MS-10 F12, U.S. Nuclear Regulatory Commission, 11545 Rockville Pike, Rockville, MD 20852-2738.

40. J. R. Jolicoeur, Office of Nuclear Regulatory Research, MS-T10 K8, U.S. Nuclear Regulatory Commission, 11545 Rockville Pike, Rockville, MD 20852-2738.

41. M. P. Kissane, IRSN/DPAM/SEMIC, Cadarache-bat 702, BP3, 13115 St-Paul-lez-Durance, France.

42. C. Kling, Westinghouse, 1344 Beulah Rd., Pittsburgh, PA 15235

43. R. Lee, Office of Nuclear Regulatory Research, MS-T10 K8, U.S. Nuclear Regulatory Commission, 11545 Rockville Pike, Rockville, MD 20852-2738.

44. C. H. Lui, Office Nuclear Regulatory Research, MS-10 K8, U.S. Nuclear Regulatory Commission, 11545 Rockville Pike, Rockville, MD 20852-2738.

45. J. E. Lyons, Office of Nuclear Regulatory Research, MS-T10 F12, U.S. Nuclear Regulatory Commission, 11545 Rockville Pike, Rockville, MD 20852-2738.

46. S. Majumdar, Nuclear Engineering Division, Argonne National Laboratory, Building 212, Argonne, IL 60439.

47. B. Marsden, School of Mechanical Aerospace and Civil Engineering, The University of Manchester, P.O. Box 88, Manchester, M60 1QD, England, UK.

48. D. N. Miotla, NE-3/Germantown Building, U.S. Department of Energy, 1000 Independence Ave., S.W., Washington, DC. 20585-1290.

49. M. Mitchell, PBMR, 1279 Mike Crawford Avenue, Centurion, 0046, South Africa

50. D. L. Moses (ORNL consultant), 130 Clemson Drive, Oak Ridge, Tennessee 37830-7664.

51. T. J. O'Connor, NE-33/Germantown Building, U.S. Department of Energy, 1000 Independence Ave., S.W., Washington, DC. 20585-1290.

52. L. Parme, General Atomics, P.O. Box 85608, San Diego, CA 92186-5608

53. S. R. Penfield, Technology Insights, 9 Overlook Lane, Carthage, TN 37030

54. D. A. Petti, Idaho National Laboratory, P.O. Box 1625, Idaho Falls, ID 83415-3860.

55. P. Pickard, Sandia National Laboratories, P. O. Box 5800, MS-1136, Albuquerque, NM 87185-1136.

56. D. A. Powers, Sandia National Laboratories, MS-0736, P.O. Box 5800, Albuquerque, NM 87185-0736.

57. P. G. Robinson, PBMR, 1279 Mike Crawford Avenue, Centurion, 0046, South Africa

58. S. Rubin, Office of Nuclear Regulatory Research, MS-T10 K8, U.S. Nuclear Regulatory Commission, 11545 Rockville Pike, Rockville, MD 20852-2738

59. R. R. Schultz, Idaho National Laboratory, P.O. Box 1625, 2525 Fremont, Idaho Falls, ID 83415-3890.

60. F. Sharokhi AREVA, Framatome ANP, 3315 Old Forest Road, P O Box 10935. Lynchburg, VA 24506

61. B. W. Sheron, Office of Nuclear Regulatory Research, MS-10 F12, U.S. Nuclear Regulatory Commission, 11545 Rockville Pike, Rockville, MD 20852-2738. 
62. M. Srinivasan, Office of Nuclear Regulatory Research, MS-T10 M05, U.S. Nuclear Regulatory Commission, 11545 Rockville Pike, Rockville, MD 20852-2738.

63. J. L. Uhle, Office of Nuclear Regulatory Research, MS-10 M5, U.S. Nuclear Regulatory Commission, 11545 Rockville Pike, Rockville, MD 20852-2738.

64. K. Weaver, Idaho National Laboratory, P.O. Box 1625, MS-3850, Idaho Falls, ID 83415-3850.

65. T. Y. C. Wei, Nuclear Engineering Division, Building 208, Argonne National Laboratory, 9700 S. Cass Ave., Argonne, IL 60439.

66. R. P. Wichner (ORNL consultant), 104 Burgess Lane, Oak Ridge, TN 37830.

67. W. Windes, Idaho National Laboratory, P.O. Box 1625, 2525 Fremont, Idaho Falls, ID 83415.

68. S. A. Wright, Sandia National Laboratories, P. O. Box 5800, MS-1146, Albuquerque, NM 87185-1146. 